\title{
EXTRACTION AND CHARACTERIZATION OF LIPIDS FROM MICROALGAE GROWN ON MUNICIPAL WASTEWATER
}

A Master's Thesis Presented to the Faculty of California Polytechnic State University

San Luis Obispo

\author{
In partial fulfillment of \\ the Requirements for the Degree of \\ Master of Science in \\ Civil and Environmental Engineering
}

by

Matthew William Hutton

October, 2009 


\title{
Authorization for Reproduction of Master's Thesis
}

\author{
EXTRACTION AND CHARACTERIZATION \\ OF LIPIDS FROM MICROALGAE GROWN \\ ON MUNICIPAL WASTEWATER
}

I grant permission for the reproduction of this thesis in part or in its entirety, without further authorization from me, on the condition that the reproducing agency provides proper acknowledgement of authorship.

Matthew Hutton

Date 


\section{Approval Page}

\section{EXTRACTION AND CHARACTERIZATION OF LIPIDS FROM MICROALGAE GROWN ON MUNICIPAL WASTEWATER}

Matthew Hutton

Submitted:

Committee Chair: Tryg Lundquist, Ph.D.

Date

Committee Member: Corinne Lehr, Ph.D.

Date

Committee Member: Yarrow Nelson, Ph.D.

Date 


\section{Abstract \\ EXTRACTION AND CHARACTERIZATION OF LIPIDS FROM MICROALGAE GROWN ON MUNICIPAL WASTEWATER}

Based on results of its Aquatic Species Program (1978-1996), which sought to develop algae-to-liquid fuel technology, the U.S. Department of Energy has suggested that algal wastewater treatment may be incorporated into biodiesel production schemes to reduce the operating costs of both processes. The purpose of the current research was to evaluate the triglycerides produced by wastewater-grown algae for their suitability as a fuel feedstock and to investigate the effectiveness of several solvent mixtures and extraction procedures at recovering lipids from fresh algae. The research involved two separate experiments. The first determined the quantity and quality of lipids produced over the lifetime of a batch culture of algae grown in a small, outdoor high-rate pond. Samples were taken regularly from an algae culture and an adaptation of the classic Bligh and Dyer extraction procedure was used to recover lipids from them. Lipids extracted from the algae samples were also analyzed by mass spectrometry for triglyceride content. Transesterification of the algal triglycerides yielded mostly saturated and monounsaturated 16 and 18-carbon fatty acids, together comprising approximately 8 to $30 \%$ of the biomass in the pond. These compounds are similar in chemical structure to conventional biodiesel feedstock compounds. The average triglyceride production rate during the growth phase of the culture was 0.97 grams per square meter of pond surface per day. A peak triglyceride production rate of $4.40 \mathrm{~g} / \mathrm{m}^{2} /$ day, or about $49 \mathrm{~L} / \mathrm{ha} /$ day, 
occurred between the eleventh and thirteenth days of batch operation, during the linear growth phase. The second experiment compared several industrially practicable extraction procedures to the Bligh and Dyer laboratory extraction method. The Bligh and Dyer procedure provides excellent lipid recovery efficiency, but several factors limit its potential on an industrial scale. The Bligh and Dyer method requires a larger volume of solvents than other methods, uses the probable carcinogenic chemical chloroform, and involves a complex series of steps that are difficult to automate. Common industrial extraction procedures use various mixtures of short-chain alcohols and alkanes. To investigate the effectiveness of scalable extraction methods, laboratory-scale tests were conducted using several different combinations of methanol, ethanol, isopropanol and hexane. The experimental extractions were performed in parallel with Bligh and Dyer extractions for comparison. The methanol solvent system removed the greatest mass of lipids, at $84 \%$ of the Bligh and Dyer extracted mass, followed by ethanol (54\%) and isopropanol (49\%). Despite recovering the smallest mass of lipid material, the isopropanol removed the largest mass of triglycerides at $83 \%$ of the Bligh and Dyerextracted mass, followed by ethanol (35\%) and methanol (23\%). In principle, given the favorable productivity and triglyceride composition of the waste-grown algae, biodiesel feedstock production could be a byproduct of algae-based wastewater treatment processes. 


\section{Acknowledgments}

I would like to express my gratitude to the some of the individuals and organizations that made this project possible.

To Dr. Tryg Lundquist, the hardest-working professor I've ever known: thank you for your enthusiasm, advice and patience. It has been a true pleasure to study, travel and work with you.

To Dr. Corinne Lehr, who has boldly undertaken the arduous task of teaching analytical chemistry to an engineer: thank you. I have learned so much from you.

To Dr. Yarrow Nelson, whose precise teaching style makes biochemical engineering seem simple: your eagerness to teach and sense of humor are genuinely appreciated.

To Ian Woertz: your earlier research and continued support made this project possible. Thank you for all of your hard work and advice.

To undergraduate investigators Stella Tan and Jennifer Zihla: thank you for your time, energy and friendship.

To my lab-mates and fellow graduate students Jeff Audett, Joe Heavin, Chris Malejan, Mike Podevin, Matt Porter, Ruth Spierling and Paul Ward: it has been a wonderful experience working with all of you. You made lab work fun despite the frigid, windy and noisy climate of the breeze. You acquiesced after my constant requests to play football in the parking lot. You worked through project after project and test after test alongside me and you taught me just as much as our professors. Thank you.

To my family, my most important teachers of all, whose love and understanding have seen me through two decades of formal education: thank you for being an unending source of inspiration.

Thank you to the California Central Coast Research Partnership and the United States Office of Naval Research for supporting algae research at Cal Poly. 


\section{Table of Contents}

Authorization for Reproduction of Master's Thesis ................................................... ii

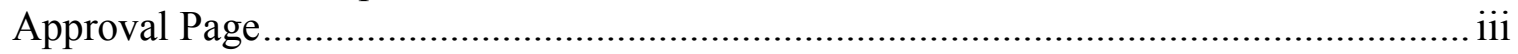

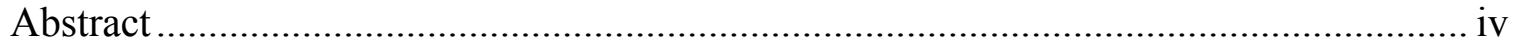

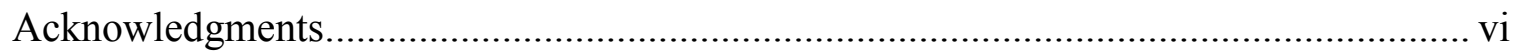

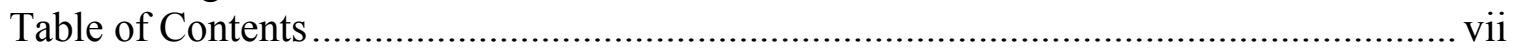

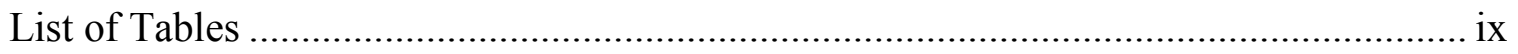

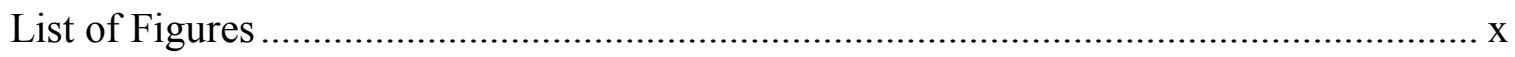

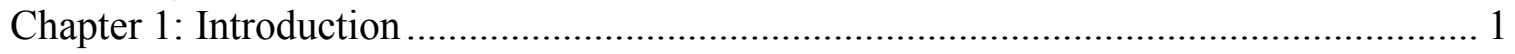

Current Energy Economy .................................................................................... 1

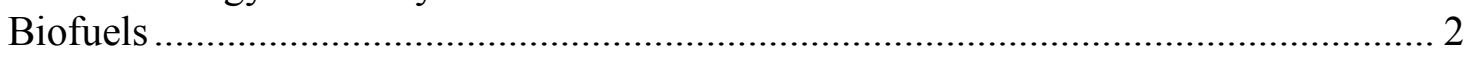

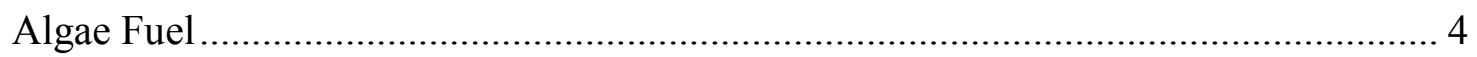

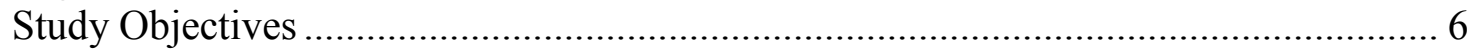

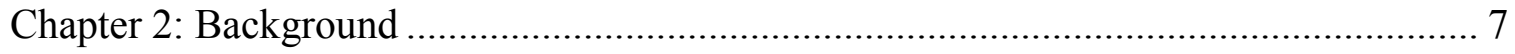

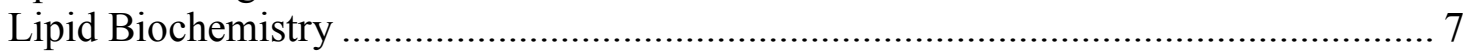

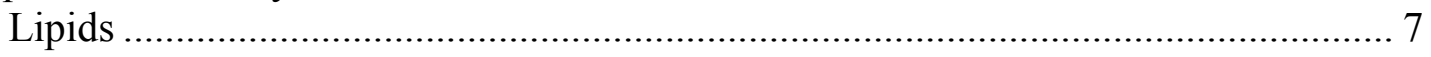

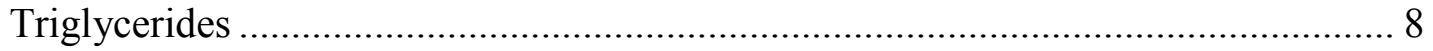

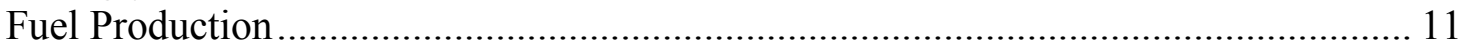

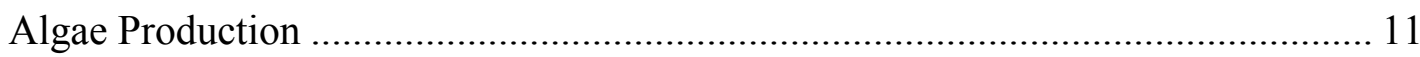

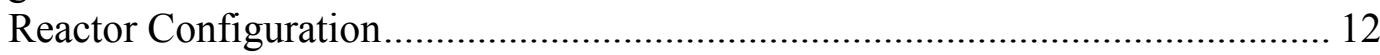

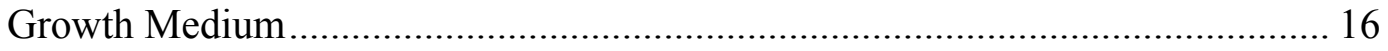

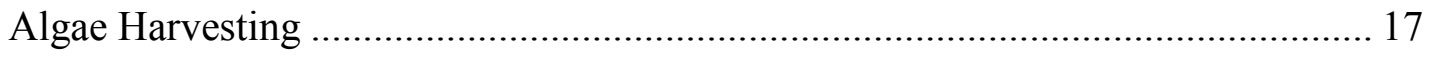

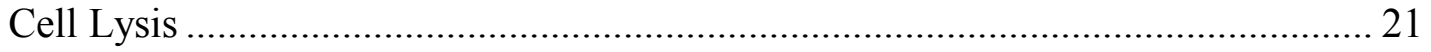

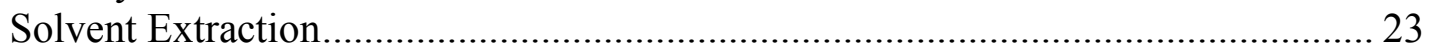

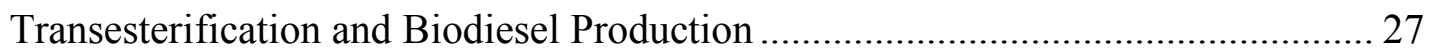

Lipid Quantification and Characterization............................................................... 28

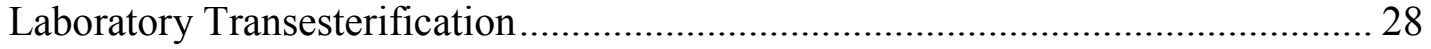

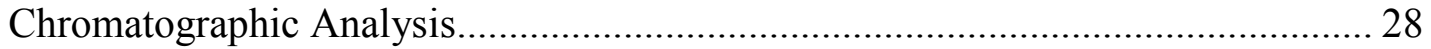

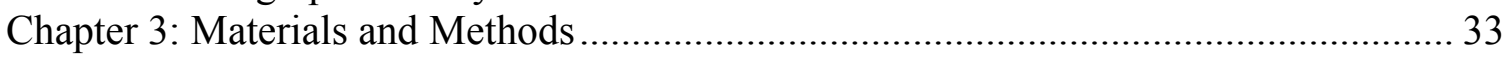

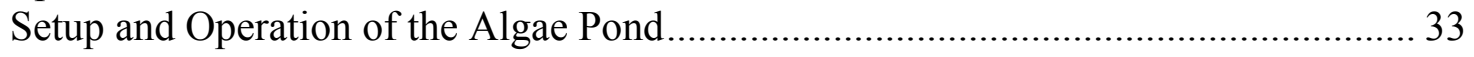

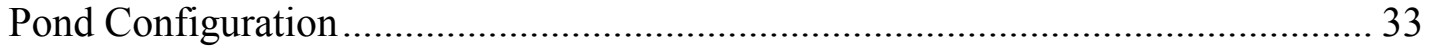

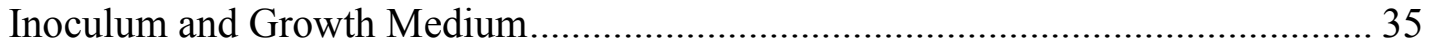

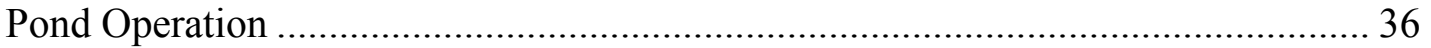

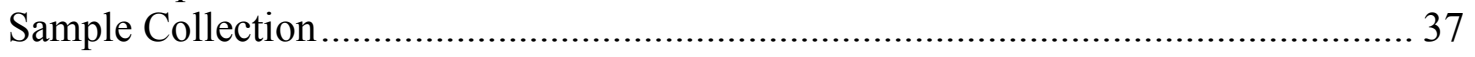

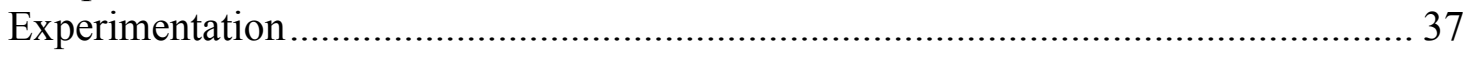

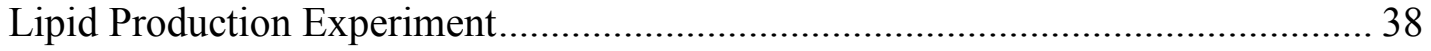

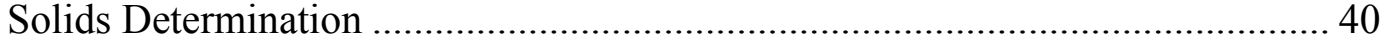

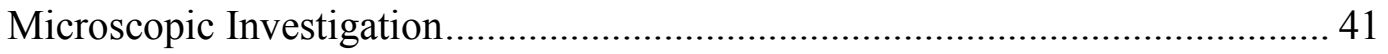

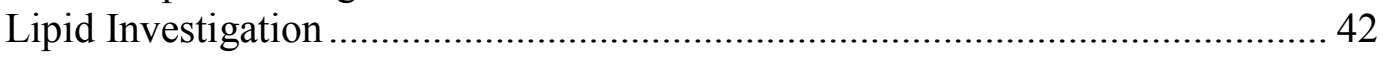

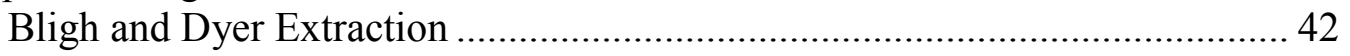

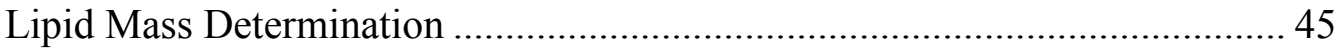

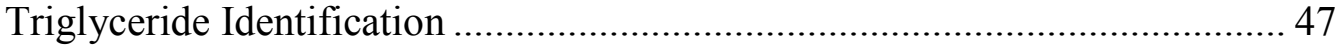

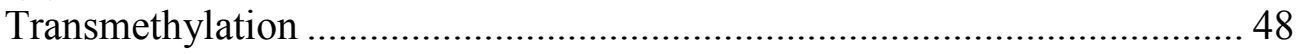


Gas Chromatography-Mass Spectroscopy ……………............................. 50

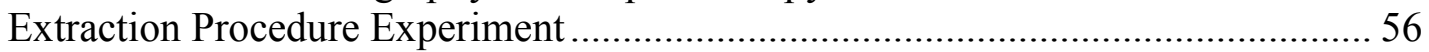

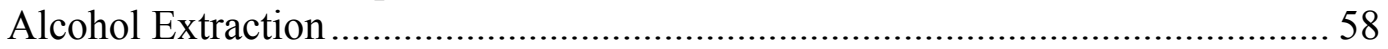

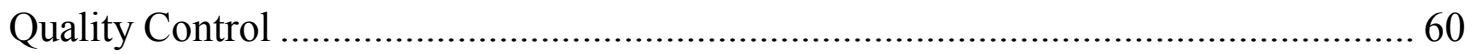

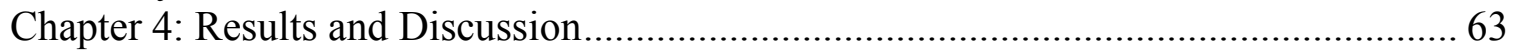

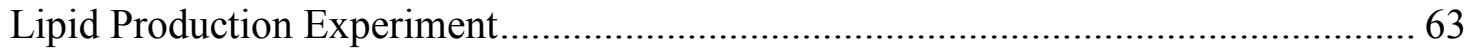

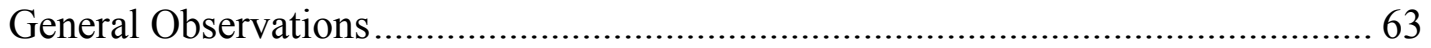

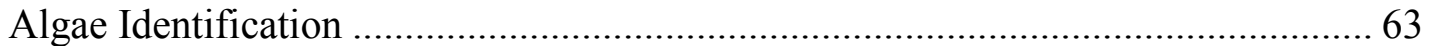

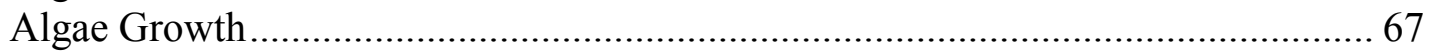

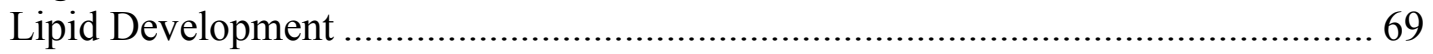

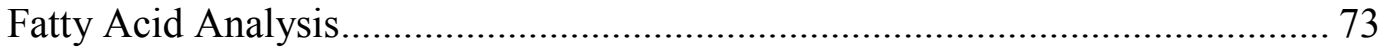

Inoculum and Growth Medium..................................................................... 73

Fatty Acids throughout Batch Growth .......................................................... 74

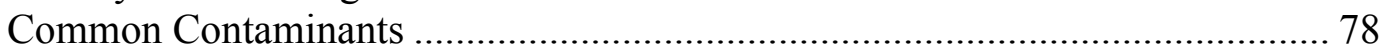

Extraction Procedure Experiment ………………………..................................... 79

Extraction Effectiveness by Solvent System ....................................................... 79

Individual Fatty Acid Methyl Esters Extracted by Each Solvent System ................. 80

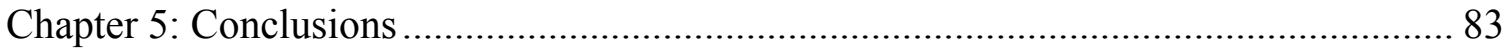

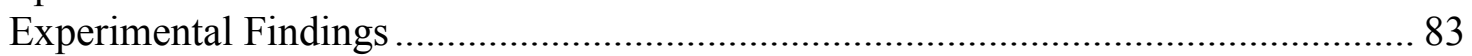

Lipid Production Experiment .......................................................................... 83

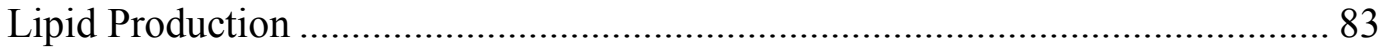

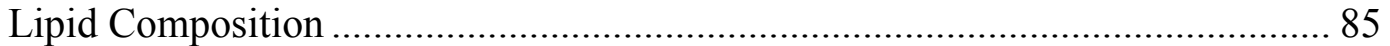

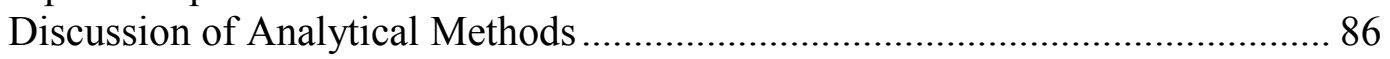

Extraction Procedure Experiment ..................................................................... 87

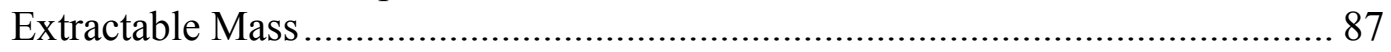

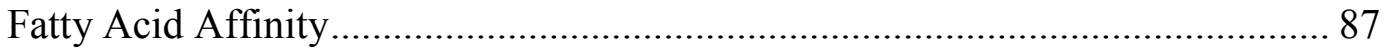

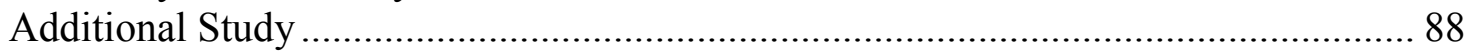

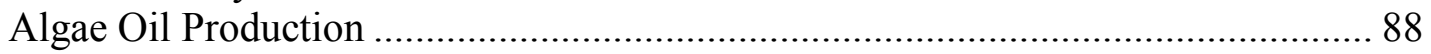

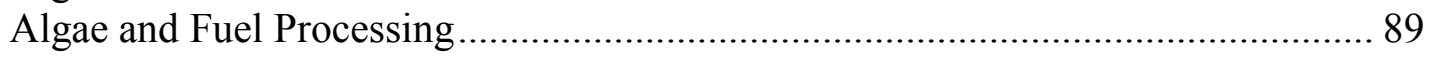

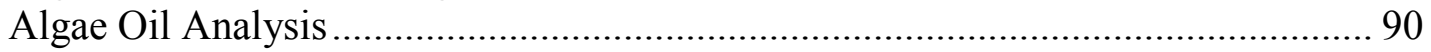

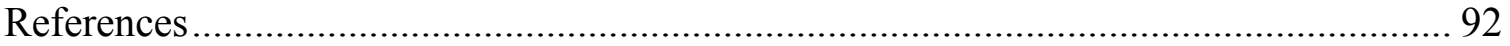

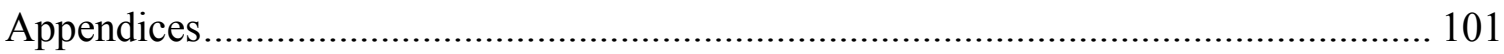

Appendix A: Lipid Production Experiment Data ................................................... 101

Appendix B: Extraction Procedure Experiment Data ................................................ 108

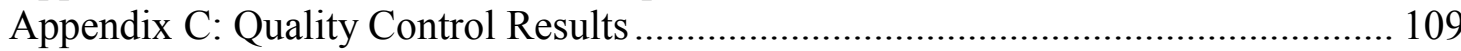

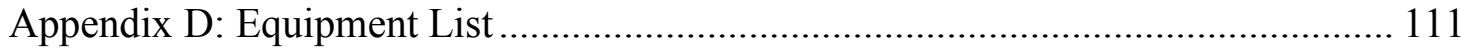




\section{List of Tables}

Table 1: Power Consumption of Several Dyno-Mills (Doucha \& Livansky, 2008) ........ 22

Table 2: Dipole moments of organic solvents (Newton, 2009) .....................................58

Table 3: Fatty acids in inoculum and initial wastewater growth medium ...................... 74

Table 4: Individual fatty acids as fraction of whole .................................................... 78 


\section{List of Figures}

Figure 1: Historical U.S. crude oil production (EIA, 2009) .......................................... 2

Figure 2: Conventional nomenclature of double-bonded carbon atoms .......................... 9

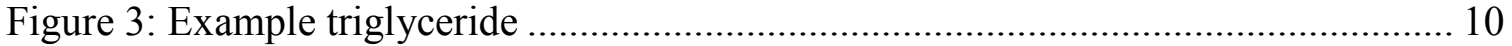

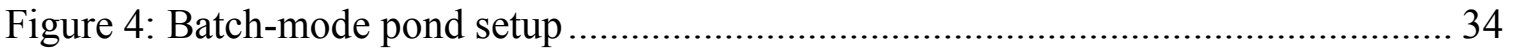

Figure 5: Weather throughout pond experiment .......................................................... 36

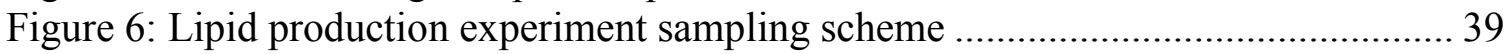

Figure 7: Bligh and Dyer extraction with chloroform layer at bottom ........................... 44

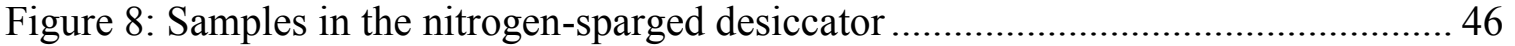

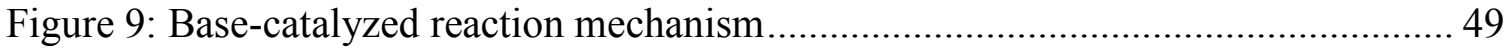

Figure 10: Gas chromatograph program .................................................................. 51

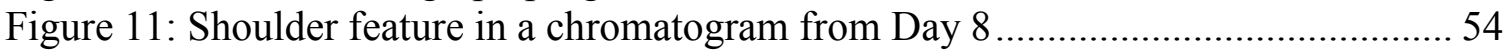

Figure 12: Head-to-tail comparisons of common constituents to reference chromatograms 55

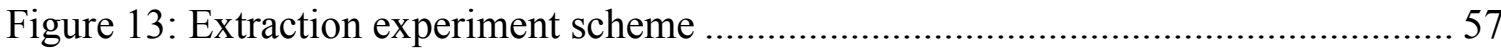

Figure 14: Alcohol-hexane extractions (methanol, ethanol, isopropanol, left to right)... 60

Figure 15: 400X Micrograph of primary wastewater effluent used in algae pond.......... 64

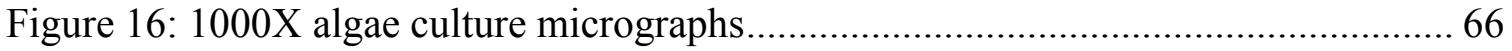

Figure 17: Total and volatile suspended solids development of batch culture ................ 68

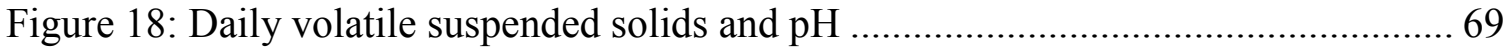

Figure 19: Comparison of gravimetric and chromatographic methods .......................... 71

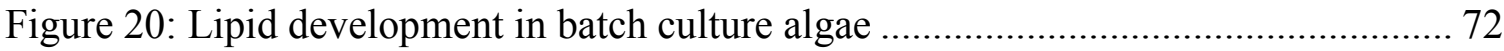

Figure 21: Fatty acid development in batch culture algae ......................................... 72

Figure 22: C16 and C18 fatty acids as percentage of volatile suspended solids ............. 75

Figure 23: Typical chromatogram of a transmethylated algal extract with internal

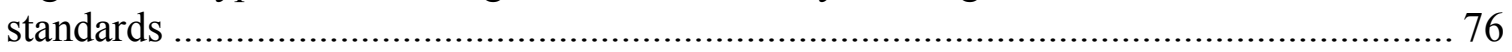

Figure 24: Concentration of fatty acids in wastewater medium during batch growth...... 77

Figure 25: Extraction effectiveness of each solvent system ....................................... 80

Figure 26: Fatty acid methyl esters extracted by solvent system.................................. 82 


\section{Chapter 1: Introduction}

\section{Current Energy Economy}

In the early 1850 s, American entrepreneurs began to develop a successful domestic oil industry. Over the next hundred years, the rapid growth of this industry fueled the transformation of America from an agrarian nation into a highly industrialized global superpower. In the process of becoming an international center of petroleum production and related technological innovation, the United States established itself as both the largest producer and consumer of fossil fuels (The Paleontological Research Institution, 2009). However, a variety of events such as the oil shocks of the early 1970 s and the climate concerns of the present have made it clear that the continued reliance on fossil fuels is at odds with the country's economic, diplomatic and environmental interests.

The economic vitality of the United States is now threatened by any disturbance in the availability of large quantities of inexpensive energy (Michael Mussa, 2000). Since the 1970s, domestic oil production in the United States has been declining (Figure 1), leading to an increased rate of oil importation (United States Energy Information Administration, 2009). This condition is seen as economically and politically unfavorable because it puts the United States in a position of dependency on other nations. 


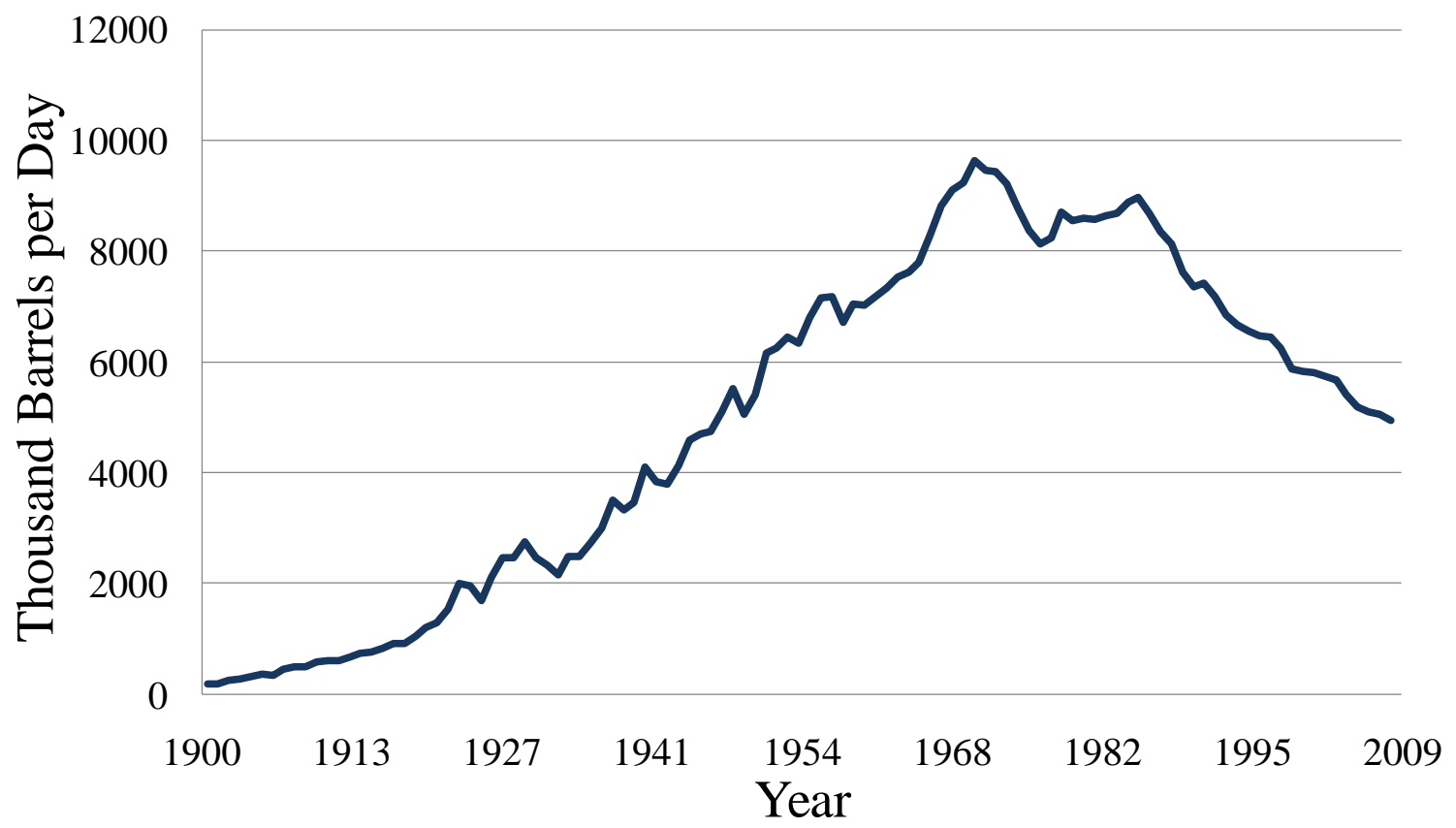

Figure 1: Historical U.S. crude oil production (EIA, 2009)

Evidence continues to mount that our reliance on fossil fuels comes at a high cost to the environment. The carbon dioxide emissions associated with fossil fuel use are considered a driving factor of the climatic warming trend currently observed throughout the world (Intergovernmental Panel on Climate Change, 2008). The large size and high rate of energy expenditure of the United States make it one of the world's largest emitters of carbon dioxide; second only to China in recent years (United States Energy Information Administration, 2006).

\section{Biofuels}

The expanded use of biofuels in the United States has the potential to alleviate many of the problems with the current energy economy. Because feedstocks can be grown 
domestically, the replacement of conventional fuels with biofuels can decrease the reliance of the United States on foreign fuel supplies.

Biofuels also may be a critical component of national efforts to reduce greenhouse gas emissions. Growing plants fix carbon dioxide into biomass, meaning that biofuels derived from plants can be combusted without any net addition of carbon to the atmosphere.

Between 2003 and 2007, the contribution of biofuels to total United States energy consumption increased from about $0.4 \%$ to about $1.0 \%$ (Energy Information Administration, 2009). In the same timeframe, biodiesel has grown from $0.48 \%$ of total biofuel energy consumption to $6.05 \%$ (Energy Information Administration, 2009). The increasing use of biodiesel, in particular, has prompted a great deal of scrutiny concerning the benefits and disadvantages of its widespread application.

Biodiesel produced in the United States is derived primarily from soy and rapeseed (Energy Information Administration, 2007). Several problems with the continued development of these feedstocks have recently become apparent. Soy biodiesel and rapeseed biodiesel can be produced at rates of about 48 and 124 gallons per acre per year, respectively (B. Greg Mitchell, 2009). At this rate, it would require approximately 2.6 million acres of the most productive rapeseed crops to satisfy the current demand of 320 million gallons per year (2008) for biodiesel in the United States (Energy Information Administration, 2009).

In addition to the large land areas required to produce significant quantities of biofuel from conventional crops, the energy benefit of soy biodiesel production has been 
questioned. Some studies have indicated that, because of the energy intensity of soy cultivation, the energy required to produce soy biodiesel is greater than the energy produced by the combustion of the biodiesel itself (Pimentel \& Patzek, 2005). Both soy and rapeseed-based biodiesel would require a large area of arable land to be produced in significant volumes. Growing world populations have led to the highest rate of human malnourishment in recorded history, leading some to argue that all available arable land should be used for food production (Pimentel \& Patzek, 2005).

\section{Algae Fuel}

It has been suggested by the U.S. National Renewable Energy Laboratory (NREL) that lipid-rich species of microalgae are a promising potential feedstock for large-scale biodiesel production (Sheehan, Dunahay, Benemann, \& Roessler, 1998). Algae assemble certain lipids as a method of energy storage. These lipids can be harvested and converted into biodiesel (Sheehan, Dunahay, Benemann, \& Roessler, 1998). Previous research has concluded that microalgae may be up to 40 times more productive a biodiesel feedstock per unit area than conventional terrestrial crops (Sheehan, Dunahay, Benemann, \& Roessler, 1998). The production of algae does not require high quality land, like most terrestrial crops. It can be grown in arid environments and many species are capable of growth in saline waters (U.S. Department of Energy, 2009). Research conducted by the U.S. Department of Energy Aquatic Species Program suggests that the costs of algae production and processing currently prohibit the use of algae as a feedstock for biodiesel fuel; however, a conclusions of the Aquatic Species Program close-out report was that 
algal wastewater treatment might be effectively combined with algae biodiesel production to reduce the cost (Sheehan, Dunahay, Benemann, \& Roessler, 1998).

Several hurdles must be overcome in order for large-scale algae biodiesel production to become a reality. First, additional research is needed to determine the reactor systems and substrates best suited to algae oil production. Although areal algae productivity rates are widely cited in current literature, relatively few studies have been conducted that track areal lipid productivity (Woertz, 2007). Similarly, until recently, little has been done to characterize the types of lipids that algae can produce, instead of simply the quantity (U.S. Department of Energy, 2009). The lack of data is especially acute for algae grown on wastewater. The types of oil produced by algae are likely to vary with growth conditions and substrates (Piorrek, Baasch, \& Pohl, 1984), (Hayakawa, et al., 2002), and should be investigated in depth.

Second, additional research is needed to develop scalable processing techniques for algal biodiesel production. The steps involved in extracting lipids from algae are complex and energy intensive (Raymond, 1983), (U.S. Department of Energy, 2009). The conventional laboratory extraction procedure, Bligh and Dyer, requires a very high solvent to biomass (vol/vol) ratio, uses highly toxic solvents which limit the usefulness of residual algae solids as a fertilizer, involves a complex choreography of steps which does not lend itself to automation, and requires a high energy input for solvent recovery. 


\section{Study Objectives}

The purpose of the current study is to address the lack of existing information on the quantity and quality of lipids that can be derived from algae in a cost effective, safe, large scale process. Specifically, this thesis presents data on the lipids produced by algae grown on municipal wastewater. These data are limited to algae collected from a single reactor and are intended to provide preliminary information on algal triglycerides, to be corroborated by further investigation.

This thesis also presents information on the effectiveness of several scalable extraction processes for recovering lipids from the algae. This information is meant to provide information about which types of extraction processes may be suitable candidates for further study.

Proximate goals of this research included:

(1) Develop a protocol for the qualitative analysis of algae triglycerides

(2) Evaluate the quantity and identity of triglycerides produced by algae grown on municipal wastewater over the course of a batch growth cycle

(3) Compare several methods of extracting oil from algae on the basis of the following:
a. Extracted total lipid mass
b. Triglyceride content of extract 


\section{Chapter 2: Background}

\section{Lipid Biochemistry}

\section{Lipids}

Many species of microalgae produce lipids. The types and quantities of lipids vary among species, and certain types of lipids can be converted into a liquid fuel product. Other types of lipids contain components that limit their utility in fuel production.

Lipids are a broad class of biomolecules which include a wide variety of compounds used in many different biological processes. Two fundamental categories of lipids are neutral lipids and polar lipids. The meaning of polarity will be discussed below in the section entitled Solvent Extraction. Neutral lipids are nonpolar and water-insoluble, whereas polar lipids have one or more water-soluble functional group. The distinction between these two categories of lipids is important because neutral lipids can be converted readily into a biodiesel fuel, while polar lipids cannot.

Neutral lipids include some complexes of oleaginous fatty acids used by cells for energy storage. A reserve of storage lipids allows some algae to respire during extended periods of light limitation and nutrient availability. Some types of algae, notably blue-green algae (cyanobacteria), do not produce neutral storage lipids (Orcutt, Parker, \& Lusby, 1986). 
Polar lipids play a critical role in membrane composition and physiological signaling. The presence of water-soluble components in polar lipids makes them unsuitable for fuel production (Sheehan, Dunahay, Benemann, \& Roessler, 1998).

\section{Triglycerides}

A triglyceride is a specific type of neutral, energy storage lipid composed of a glycerol molecule esterified with three fatty acids. Triglycerides vary widely in fatty acid composition. Generally, fatty acids range from 4 to 30 carbon atoms in length (Ophardt, 2009). Carbon chains of even-numbered length are predominant because the de novo biosynthesis of fatty acids involves a two-carbon acetate ion and the subsequent chain elongation is carried out by the donation of two-carbon units from malonyl-coA (Gunstone, 1996).

A saturated acid is one in which there are no double bonds along the carbon chain. The main carbon chain of a saturated fatty acid is saturated with hydrogen atoms. Molecules that include only one carbon-carbon double bond are said to be mono-unsaturated. Molecules that include more than one carbon-carbon double bond are said to be polyunsaturated (Bailey, 2000).

Double-bonded carbon atoms within a fatty acid vary in terms of geometric isomerism and can be arranged in either of two different conformations. The International Union of Pure and Applied Chemistry, IUPAC, has adopted a standard nomenclature to describe the different conformations. Molecules in which similar functional groups are on the same side of the carbon chain are called cis-bonded. Molecules in which similar functional groups are on opposite sides of the carbon chain are called trans-bonded. 
These terms are abbreviated as $\mathrm{Z}$ for cis-type bonds and $\mathrm{E}$ for trans-type bonds. An example of the naming conventions is illustrated in Figure 2 (Bailey, 2000).

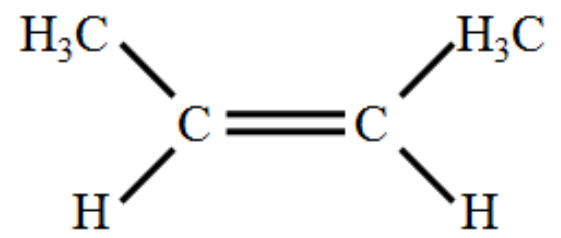

Cis-2-Butene But-2-ene (Z)

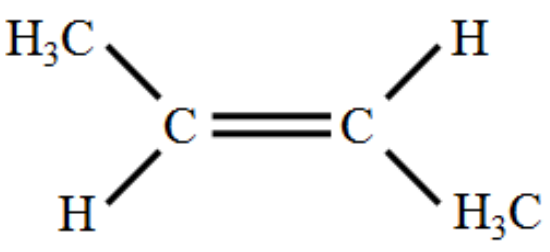

Trans-2-Butene But-2-ene (E)

Figure 2: Conventional nomenclature of double-bonded carbon atoms

Fatty acids and fatty acid methyl esters are frequently described using a shorthand notation which has been adopted by IUPAC (Bailey, 2000). The abbreviated notation takes the form $\mathrm{CX}: \mathrm{Y}$, where $\mathrm{C}$ stands for carbon, $\mathrm{X}$ is the number of carbon atoms in the main carbon chain, including the atom in the carbonyl group, and $\mathrm{Y}$ is the number of carbon-carbon double bonds in the carbon chain (IUPAC, Commision on the Nomenclature of Organic Chemistry, 1979).

Glycerol, also known as glycerin or glycerine, is an organic trihedral alcohol with the formula $\mathrm{C}_{3} \mathrm{H}_{5}(\mathrm{OH})_{3}$. Glycerol is used by cells to link fatty acids together for storage. It is also a byproduct of biodiesel production.

Triglycerides play a central metabolic role in organisms as a means of energy storage and transportation (Bailey, 2000). In a fuel, the energy stored within the carbon-carbon and carbon-hydrogen bonds of these molecules can also be released to do mechanical work in 
an engine. Triglycerides are of widely variable composition, even within an organism. A triglyceride typical of algae is pictured below. Although triglycerides are most easily visualized with their three fatty acids chains in parallel, in reality, triglycerides are not arranged in this manner. The fatty acids of a triglyceride repel each other, resulting in a molecule arranged as a central glycerol group, with three acids radiating outward.

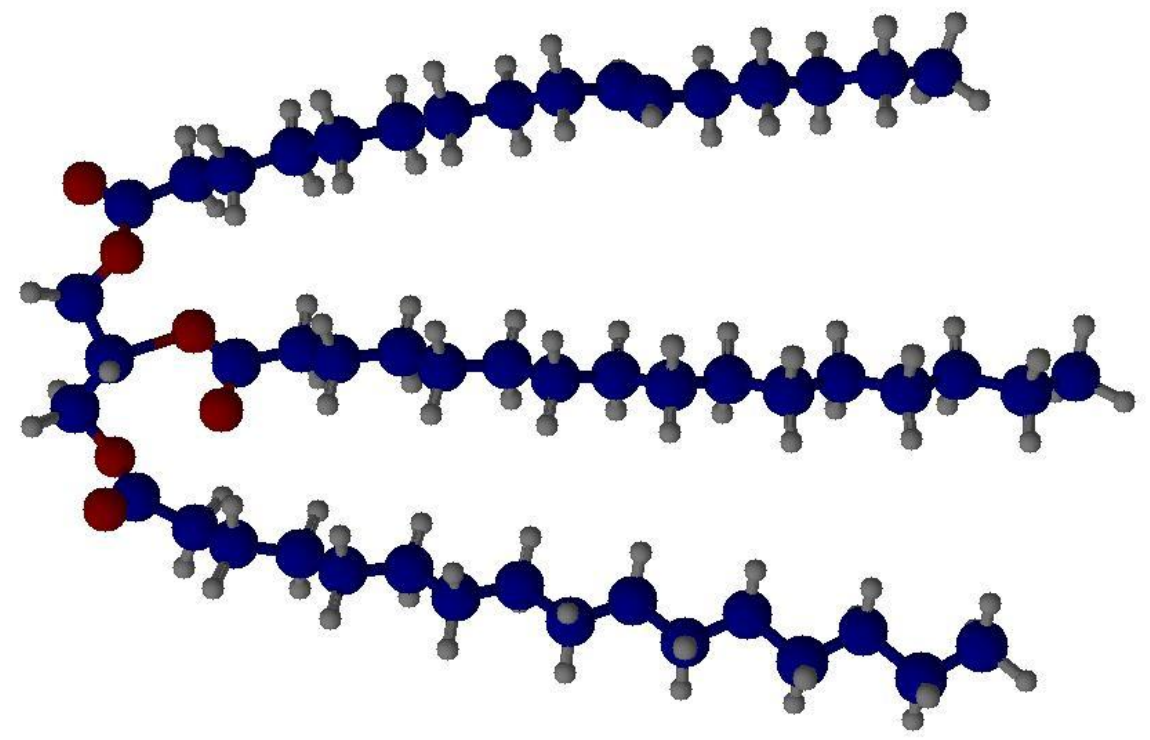

Figure 3: Example triglyceride

In biofuel-related literature the term lipids is commonly used interchangeably with the term triglycerides. Some characteristics common to various types of lipids make it difficult to isolate triglycerides for biofuel processing. For example, both triglycerides and sterols are types of nonpolar lipids. However, triglycerides are the only molecules from which biodiesel can be produced directly. 


\title{
Fuel Production
}

In this section, some background information on the overall algae-to-fuel production process is presented in the sequence of unit operations envisioned for an algae-to-fuel production scheme. Integrated algae biofuel systems were first proposed by Oswald and Golueke (Oswald \& Golueke, 1960). These early investigators considered only biogas production from algae, not the combined lipid and biogas production now commonly considered the default algae fuel production system (U.S. Department of Energy, 2009). The current theoretical process scheme includes similar unit operations to a conventional oil crop biofuel production scheme.

\section{Algae Production}

\begin{abstract}
Algae must be grown space-efficiently, energy-efficiently and cost effectively if they are to be used to produce fuel on an industrial scale. Arable land is in high demand and a successful biofuel crop cannot require too much of it to grow (Pimentel, 2003), (Gardner $\&$ Tyner, 2007). The production of the crop and subsequent fuel conversion process must consume less energy than the biofuel can provide (Pimentel \& Patzek, 2005). Only a cost effective biofuel will compete effectively with conventional fuels at market (Haas, 2005). In algae biofuel production, these factors are influenced by the cell density and growth rate of algal culture, which are in turn controlled in large part by reactor configuration and nutrient supply (Benemann, Koopman, Weissman, Eisenberg, \& Goebel, 1980), (Tedesco \& Duerr, 1989).
\end{abstract}




\section{Reactor Configuration}

The configuration of an algae-producing reactor can be generally described by specifying whether it is open or closed and whether it is operated in batch or continuous mode. The terms open and closed refer to the level of interaction between the algal culture and surrounding environment. Batch and continuous refer to the duration of time growth media remain in a reactor (Shuler, 2002).

A closed system partially isolates algae by circulating growth media through a system of tubes or other containers, whereas open systems typically consist of uncovered ponds, directly exposed to the elements (U.S. Department of Energy, 2009). The tubes in a closed reactor must be transparent to accommodate photosynthesis, and so these systems are known as photobioreactors (Chaumont, 1993). An open system consists of a shallow channel or pool. In an open system, the surface of the growth media is directly exposed to the surrounding environment. This type of system is known as a pond. In a special type of pond, called a raceway pond or high-rate pond, growth media is circulated to provide mixing (Sheehan, Dunahay, Benemann, \& Roessler, 1998). There are advantages and disadvantages of each type of system.

One advantage of closed photobioreactors is that they discourage culture contamination and culture escape. The latter is extremely important if genetically modified algae are to be used for industrial fuel production. Because algal growth media in a photobioreactor is isolated from its surroundings, the introduction of foreign species by water fowl, aeolian transport and other common vectors is minimized. Photobioreactors are popular tools for researchers investigating the characteristics of pure algae cultures. However, the 
scale-up of photobioreactor systems is complicated high capital and operating costs. To date, efforts to grow large quantities of algae in photobioreactors have been unsuccessful (Benemann, 2008).

The cost of constructing a large-scale photobioreactor system is high relative to comparably sized open pond system (Benemann, 2008). A large quantity of expensive transparent material is required to build a photobioreactor. The equipment used to circulate media through a photobioreactor is particularly expensive (Weissman, Goebel, \& Benemann, 1998), (Sheehan, Dunahay, Benemann, \& Roessler, 1998). In-line pumps and fittings make a significant contribution to capital costs. Because photobioreactor systems are enclosed, cooling equipment is necessary in order to prevent heat damage to algae (Tredici \& Zittelli, 1998), (U.S. Department of Energy, 2009).

The operational costs of a photobioreactor include pumping, cleaning, cooling and maintenance. Pumping power is provided to circulate media through the system, and adds energy costs. Cleaning costs are associated with the growth of algae on the internal surfaces of the system. Algae that attach themselves to the transparent surfaces prevent light from penetrating the entire depth of growth media. This shading limits the productivity of the system and must be mitigated by regular cleaning. Systems that scrape tubular sections or pump abrasive granular media through photobioreactors add to operational costs. Because heat cannot be dissipated from a closed system by evaporation of growth media to the atmosphere, cooling demands must be addressed with expensive heat exchangers (U.S. Department of Energy, 2009). 
The advantages of pond systems include low capital and operating costs relative to closed systems of similar capacity. The construction of a pond system involves excavating an area to a depth of up to a meter. This cost is modest compared to the construction of a closed system of the same volume (U.S. Department of Energy, 2009). In raceway ponds, mixing is provided by large paddle wheels. Cooling equipment is not required, since the temperature of a pond system is moderated by the evaporation of process water. However, if pond heating were necessary, expensive heat exchangers would be required.

The operational costs of a pond system are low because ponds do not require a great deal of maintenance. Energy costs of mixing constitute a large portion of the costs (Weissman, Goebel, \& Benemann, 1998). The major disadvantage of ponds is the likely increased difficulty in controlling invasive algae species and pests, such as algae-grazing zooplankton (Rodolfi, et al., 2008).

Regardless of whether they are open or closed, reactors can be operated in a number of different modes. In continuous mode, influent is constantly discharged into the ponds, and algae-laden water is constantly removed from the ponds at the same rate. When operated in continuous mode, raceway ponds in California have a hydraulic residence time ranging from two to ten days, depending on climate, season and purpose (Oswald, 1990), (Belay, A. pers. comm., 2009).

In batch operation, a reactor is filled with a growth medium, inoculated with algae, and then left to grow. Because no additional nutrients are introduced during batch growth after inoculation, the nutrient concentration can become growth-limiting. In batch growth, populations of microorganisms often grow and decay in four or five phases 
(Shuler, 2002). The first phase, called the lag phase, occurs immediately after inoculation. During the lag phase, the cell concentration remains approximately constant as cells undergo metabolic adjustments to adapt to their new environmental conditions (Shuler, 2002). The second phase is the exponential growth phase, during which the cell concentration grows geometrically (Shuler, 2002). The exponential phase is sometimes followed by a linear growth phase as the culture transitions into the stationary phase (Lundquist, T. pers. comm., 2009). During the stationary phase, the rate of cell multiplication is approximately the same as the rate of cell death, and little net change is observed in the population (Shuler, 2002). Finally, a fifth phase known as the death phase takes place, during which a progressive lack of available nutrients leads to the decline of cell concentration in the culture (Shuler, 2002).

Batch-mode operation is common in commercial algae operations (Belay, A. pers. comm., 2009). A related method also common to algae production is called semi-batch operation. In semi-batch operation only a fraction of the reactor volume is replaced during each reactor cycle (Lavens \& Sorgeloos, 1996).

Batch and semi-batch growth modes are popular among commercial operations because, in conjunction with inoculation by pure culture, they help prevent invasive species from becoming dominant in ponds (Belay, A. pers. comm., 2009). These modes of operation might also be used to create a nutrient-depleted environment for the algae. In some algal species, nitrogen starvation induces lipid production (Suen, Hubbard, Holzer, \& Tornabene, 1987), (Hu, et al., 2008), (Rodolfi, et al., 2008). Achieving nutrient depletion is possible in batch reactors because algae growth decreases soluble nutrient 
concentrations and ammonia volatilization decreases total ammonia concentrations in the growth medium (Sheehan, Dunahay, Benemann, \& Roessler, 1998).

In research, batch and semi-batch operation also make it possible to evaluate the quantity and types of lipids produced throughout the lifespan of an algal culture, which requires more equipment to ascertain using continuous cultures.

\section{Growth Medium}

The type of algal growth medium to be used is a critical consideration in algae production system designs. Algae are capable of thriving in nutrient-rich waters of a wide variety of compositions. Many of the algae oil studies reported in current literature were conducted using defined growth media (Hu, et al., 2008), (Zhu, Zong, \& H.Wu, 2008), (Rodolfi, et al., 2008). Despite some encouraging growth rates, the use of defined media, particularly those incorporating fossil fuel-derived nitrogen fertilizer, is at odds with the goal of mitigating environmentally harmful carbon dioxide emissions. Of worldwide nitrogen fertilizer production, approximately $97 \%$ is derived from synthetically produced ammonia. The energy demand associated with this production accounts for about $5 \%$ of world natural gas consumption (Food and Agricultural Organization of the United Nations, 2006).

The use of municipal wastewater to cultivate algae has several advantages over the use of conventional synthetic nitrogen fertilizer. The combination of wastewater treatment and algae production has the potential to reduce the costs for both processes, and nutrient removal can be effectively conducted in high-rate algae ponds (Sheehan, Dunahay, Benemann, \& Roessler, 1998). The algae grown in such a system can produce usable 
quantities of oil (Enssani, 1987), (Feffer, 2007). Since municipal wastewater contains various forms of bioavailable nitrogen, energy-intensive nitrogen fertilizers are not required to grow algae on wastewater. The avoidance of fossil fuel-derived nitrogen fertilizers in algae production can reduce the energy requirements of the overall process.

\section{Algae Harvesting}

The dense algae cultures possible, up to 5 grams per liter (Weissman, Goebel, \& Benemann, 1998), can translate into high oil productivities in relatively small reactor volumes (Raymond, 1983); however, the unique characteristics of algal culture also present some harvesting challenges.

Because processing the entire volume of an algae growth vessel is impractical, algal biomass must be concentrated prior to the conversion to biofuel production (U.S. Department of Energy, 2009). Because microalgae cells are small in diameter and similar to water in density, concentrating them is difficult (Benemann, Koopman, Weissman, Eisenberg, \& Goebel, 1980).

The concentration of the algal biomass simplifies each subsequent step in biofuel refining. It reduces the amount of energy required to move algae-laden water through a processing stream and lowers the quantities of solvents that must be used in order to extract oils. Growth media in mixed algae cultivation ponds systems often contains as little as $0.02 \%$ algae by mass and such cultures must be concentrated to at least $1 \%$ algae by mass in order for subsequent unit operations to be effective (U.S. Department of Energy, 2009). Concentration can be accomplished by filtration, screening, 
centrifugation, flotation or sedimentation (Benemann, Koopman, Weissman, Eisenberg, $\&$ Goebel, 1980). Coagulation and flocculation may be used to enhance any of these processes (Metcalf and Eddy, 2003).

Filtration is a mechanical separation method usually using a bed of granular media or a porous membrane. Cloth media can also be used, as in the case of a rotary drum filter (Raemy, 2008). In a rotary drum system, filter material covers the circumference of a drum, which rotates, partially submerged in the algae-laden water. The application of a vacuum to the inside of the drum allows submerged sections to collect solid biomass, while sections exposed to air are scraped clean (Dodd, 1981), (Shuler, 2002). However, it is unlikely that a filtration process could be effectively used in a algae biofuel process stream (Benemann, Koopman, Weissman, Eisenberg, \& Goebel, 1980). Although relatively large organisms such as Spirulina and filamentous algae are routinely harvested by filtration or screening, most algae species observed growing in wastewater are not large enough to be removed by filtration without costly coagulation (Benemann, Koopman, Weissman, Eisenberg, \& Goebel, 1980).

Centrifugal separation involves the application of a centrifugal field to a liquid. This force causes relatively dense materials to settle more rapidly than they would under normal gravitational force. Several types of continuous centrifuges are used in industrial processes. Each variation uses a slightly different mechanism to separate dense materials from other materials (Shuler, 2002). Centrifugation is suitable for research, final thickening of slurries, and recovery of high-value products. However, it is unlikely that centrifugation could be effectively scaled up for a biofuel application due to costs and 
high energy consumption (Benemann, Koopman, Weissman, Eisenberg, \& Goebel, 1980).

Flotation is a process in which a pressurized gas is dissolved into the liquid medium. As air is released from solution, small bubbles nucleate on particles in the fluid (Metcalf and Eddy, 2003). As the bubbles rise toward the open atmosphere, they bring particles with them. At this point, a concentrated float of particles (e.g., algal biomass) can be skimmed from the top of the solution with a concentration as high as $4 \%$ solids (MWH, 2005).

Sedimentation is a unit operation in which particles are removed from a liquid under the influence of gravity. A sedimentation vessel may have an ascending vertical hydraulic flow, a longitudinal flow or a diagonally ascending flow (e.g., lamella settlers). Particle removal by sedimentation requires the downward settling velocity of the particle or floc to be greater than the overflow rate of the vessel. This requirement leads to the fundamental design parameter of a vertical settling system:

for a given particle to settle

Overflow rate, $V_{0} \leq$ Particle settling velocity, $V_{S}$

$$
V_{0}=\frac{\text { where }}{\text { Slow rate }}
$$

Although the overflow rate equation is more complex for a diagonal system, the same principle applies in both cases: for a given particle to settle, the ratio of the longitudinal dimension to the vertical dimension of the settling space must be greater 
than or equal to the ratio of the longitudinal component of the velocity of the particle to the downward component of the velocity of the particle (MWH, 2005).

Coagulation and flocculation are two related processes that are often used in series to form large flocs from small, difficult-to-settle particles (Benemann, Koopman, Weissman, Eisenberg, \& Goebel, 1980), (Metcalf and Eddy, 2003). Coagulation is the addition of a chemical additive called a coagulant to a process-water in order to neutralize the net charge on the surface of suspended particles. A coagulant has multiple charged binding sites which affix to charged particles in suspension. A rapid mixing is commonly used to encourage contact between the coagulant and particles.

Flocculation occurs during a slow-mixing process that promotes the formation of particle agglomerates. The resulting large particles are separated relatively easily by sedimentation or other means (Metcalf and Eddy, 2003). Any of the aforementioned concentration techniques can be combined with coagulation and flocculation, although in some cases particles may not require artificial coagulation/flocculation to settle (Shuler, 2002).

In algae-based wastewater treatment systems, it is common to remove microalgae by a combination of coagulation, flocculation and flotation (Matt Gerhardt, 2009), (Benemann, Koopman, Weissman, Eisenberg, \& Goebel, 1980). Following algal cell harvesting and concentration, the microalgae cells may be broken and their oils extracted. 


\section{Mechanical Cell Lysis}

Cell disruption, or lysis, is the destruction of cellular membranes in order to release internal products. Prior to lipid extraction, algal cells must be disrupted to facilitate release of the lipids (U.S. Department of Energy, 2009). Because microalgae have strong, rigid cell walls, their disruption is more demanding than it is for other microorganisms such as bacteria (U.S. Department of Energy, 2009).

A number of cell disruption technologies are available for industrial-scale processing, including bead milling, homogenizing and sonication. In an industrial setting, an appropriate cell disruption technology is selected based on the durability of the cell walls to be disrupted, the size of the process stream, the risk of subcellular destruction of important products, the costs of the process and the safety concerns (Shuler, 2002).

A bead mill uses a large number of small, high-velocity beads to break cell walls. By exciting the beads, a bead mill produces shear forces large enough to destroy cell walls. Bead milling is traditionally a laboratory-scale process; however, a large-scale type of bead mill called a dyno-mill has been used successfully to disrupt microalgae cells. A dyno-mill excites beads using rapidly rotating, notched discs (Shuler, 2002). Although high rates of cell disruption are possible, the process of bead-milling requires a great deal of energy (Doucha \& Livansky, 2008). In spite of high energy costs, it has been proposed that dyno-milling is the most practical method of large-scale, mechanical cell disruption for algae processing (Cohen, 1999). The following table compares the rate of Chlorella cell destruction to power-to-mass ratios of several commercially available dyno-mills. 
Table 1: Power Consumption of Several Dyno-Mills (Doucha \& Livansky, 2008)

Mass flow $M$ of disrupted algae cells from disintegration chamber, power consumption $P$ and specific power consumption $P / M$ in the homogenizers Dyno-Mill

\begin{tabular}{|c|c|c|c|c|c|c|c|}
\hline $\begin{array}{l}\text { Algae dry } \\
\text { weight }\left(\mathrm{g} \mathrm{l}^{-1}\right)\end{array}$ & $\begin{array}{l}\text { Feed rate } \\
\left(\mathrm{kg} \mathrm{h}^{-1}\right)\end{array}$ & $\begin{array}{l}\text { Beads } \\
\text { filling }(\%)\end{array}$ & $\begin{array}{l}\text { Speed of agitator } \\
\text { discs }\left(\mathrm{m} \mathrm{s}^{-1}\right)\end{array}$ & $\begin{array}{l}\text { Degree of cells } \\
\text { disintegration }(\%)\end{array}$ & $\begin{array}{l}M(\text { kg (dry } \\
\text { weight) } \mathrm{h}^{-1)}\end{array}$ & $P(\mathrm{~kW})$ & $\begin{array}{l}P / M(\mathrm{kWh} \mathrm{kg} \\
\text { (dry weight) }^{-1} \text { ) }\end{array}$ \\
\hline \multicolumn{8}{|c|}{ a) Dyno-Mill KD $20 \mathrm{~S}$} \\
\hline \multicolumn{8}{|c|}{$\mathrm{ZrO} 2$ beads $0.3-0.4 \mathrm{~mm}:$} \\
\hline \multirow{3}{*}{124.2} & 35 & 85 & \multirow[t]{3}{*}{13} & 82.8 & 3.27 & 27.4 & 8.37 \\
\hline & 38 & 75 & & 75.2 & 3.22 & 24.5 & 7.60 \\
\hline & 120 & 75 & & 58.1 & 7.87 & 24.5 & 3.11 \\
\hline \multicolumn{8}{|c|}{$\mathrm{ZrO} 2$ beads $0.6-0.8 \mathrm{~mm}:$} \\
\hline 124.2 & 35 & 85 & 13 & 85.2 & 3.36 & 25.1 & 7.51 \\
\hline \multicolumn{8}{|c|}{ b) Dyno-Mill KD $25 \mathrm{~S}$} \\
\hline \multirow[t]{2}{*}{102.5} & 35 & 85 & 13 & 90.6 & 2.95 & 29.6 & 10.03 \\
\hline & 120 & & & 77.7 & 8.69 & 24.5 & 2.82 \\
\hline
\end{tabular}

Another method of disruption is called homogenization. In this process, pumps are used to accelerate the liquid medium to a high velocity. The action of the pump, itself, or the subsequent collision of the high-velocity impact ring applies shear forces to the liquid which can destroy cell walls (Shuler, 2002). Although more study is needed to document the shear sensitivity of algae cells (Joshi, Elias, \& Patole, 1996), existing investigations have shown that it is difficult to achieve high rates of cell wall destruction using a highshear homogenization system (Vandanjon, Rossignol, Jaouen, Robert, \& Quemeneur, 1999). Due to the high energy requirements of homogenization of algae, it has been proposed that this process is best suited for the recovery of high-value products rather than biofuel feedstock (Asenjo, 1990), (Doucha \& Livansky, 2008).

Ultrasonic cell disruption is used to apply ultrasound energy to a solution containing a culture of cells. At sufficiently high frequency and energy intensity, the vibration of an ultrasonic probe causes the rapid nucleation and collapse of bubbles in solution (Shuler, 
2002). Although they are much smaller than algae cells, the collapse of the bubbles creates small regions of extremely high pressure which can shear cell walls apart (Chaplin, 2004).

Ultrasonic cell disruption is an extremely effective method of destroying cell walls at laboratory scale (Chisti \& Moo-Young, 1986), (U.S. Department of Energy, 2009). Thus, it is often assumed in laboratory extractions that all algae cells in sonicated samples are sufficiently lysed to provide exposure of all cellular triglycerides to the extracting solvent. Due to the large quantities of energy required per unit volume, ultrasonic cell disruption is likely not a practical industrial-scale method of cellular disruption (Chisti \& Moo-Young, 1986).

\section{Solvent Extraction}

Solvent extraction is used to isolate components emulsified or dissolved in a mixture; such as oil in water. In simple extractions, the emulsified mixture is blended with a solvent that will dissolve targeted compounds. The separation of specific compounds is possible because of differences in solubility between the component of interest and other components of the mixture (Bailey, 2000). Since water is highly polar, the addition of a nonpolar solvent to an aqueous solution results in the formation of a biphasic mixture (Shuler, 2002). The newly formed nonpolar phase attracts nonpolar compounds, such as oils, which had previously been emulsified or partially dissolved in the aqueous solution.

Targeted compounds such as triglycerides can be isolated by elevating the solution to a temperature at which the solvents will evaporate, but the targeted compounds will not. In 
practice, solvents are recovered after vaporization and recycled. This process is not perfectly efficient and results in the loss of some fraction of the solvents (Shuler, 2002).

The chemical interactions responsible for the formation of a biphasic solution and the migration of nonpolar species into the nonpolar layer both are related to molecular structure (Bailey, 2000). In nonpolar bonds, the bonding electrons are shared equally by both atoms whereas in polar bonds, electrons are more strongly attracted to the more electronegative atom and a greater electron density is found near this atom. This dislocation of electric charge causes a dipole moment. Molecules with stronger dipole moments are more polar than molecules incorporating more nonpolar bonds (Bailey, 2000).

The polarity of a molecule is characterized by a value known as an electric dipole moment, which incorporates the difference in charge across a molecule and the distance over which the charge difference occurs (Schwartz, 1972). The standard unit of electric dipole moment is the Debye (National Institute of Standards and Technology, 2006). A Debye is approximately $3.34 \times 10^{-30}$ coulomb-meters (Rowlett, 2003).

In the formation of a solution, intermolecular forces between the solute molecules and intermolecular forces between the solvent molecules decrease and new intermolecular forces between the solvent and solute are formed. Polar substances dissolve in polar substances because it is thermodynamically favorable for the solvent-solute forces to form (Bailey, 2000). For example, in the commonly used water-nonpolar solvent extraction system, it is not thermodynamically favorable to break strong hydrogen bonds between water molecules in order to form weak dipole-induced dipole forces between the 
water and the nonpolar solvent. Therefore, the non-polar solvent does not dissolve in the polar solvent. This tendency results in a minimization of the common surface area between the water and the nonpolar solvent: ideally, a flat plane. The hydrophobicity of most nonpolar solvents is the basis of solvent extraction (Shuler, 2002).

Specific to analytical extractions, research conducted throughout the 1950 s by E. G. Bligh and W. J. Dyer of the Fisheries Research Board of Canada led to the 1959 publication of a method for the rapid extraction of lipids from fish tissues. The method uses a chloroform-methanol solvent system (Bligh \& Dyer, 1959). The Bligh and Dyer method of lipid extraction has been refined through other studies since its initial publication. For example, a modification of the Bligh and Dyer procedure specifically for lipid extractions from algae has been published (Enssani, 1987). This modified Bligh and Dyer method has been found to recover approximately $70 \%$ of total estimated lipids (Enssani, 1987). However, the industrialization of the process poses several problems. For example, the procedure requires a very high solvent to biomass (vol/vol) ratio, uses highly toxic solvents, involves a number of complex steps which does not lend itself to automation, and requires a large energy input for solvent recovery (Enssani, 1987), (Hara \& Radin, 1978).

In contrast, the ideal extraction method would be scalable, safe, inexpensive, and have a low energy requirement. This means minimizing the number and complexity of process steps, limiting the use of hazardous materials and minimizing the material and energy consumption of the process (Enssani, 1987). 
At industrial scale, various mixtures of short-chain alcohols and alkanes are more commonly used for extractions. For example, methylene chloride is frequently used to decaffeinate coffee beans (Nelson, Y. pers. comm., 2009). While, alcohol solvents do pose a fire hazard, they carry less of a risk of acute toxic exposure than the chloroform used in the Bligh and Dyer method. Most alkanes are also mildly toxic and highly flammable; but again, do not pose as serious a threat as chloroform (Hara \& Radin, 1978). Methods implemented at an industrial scale, such as caffeine removal from coffee beans, consist of fewer steps than Bligh and Dyer and do not require as much energy input per unit of production.

Although heated solvents have not been evaluated in this thesis, heated extractions may be effective for removing lipids from microalgae. Despite the increased energy demand associated with heated, they may help to break down cell walls, allowing the recovery of more lipids than a traditional solvent system would (Nagle \& Lemke, 1990).

Supercritical carbon dioxide is another extraction method which has not been considered in the current research. When carbon dioxide is heated beyond its critical temperature and pressure, it reaches a supercritical state and although it behaves like a gas, it also exhibits some characteristics of a liquid solvent (Andrich, Nesti, Venturi, Zinnai, \& Fiorentini, 2005). These technologies have not been studied exhaustively for the extraction of lipids from microalgae. 


\section{Transesterification and Biodiesel Production}

After extraction from biomass, triglyceride lipids can be converted into fuel compounds by transesterification. The transesterification of triglycerides yields biodiesel, which is a mixture of fatty acid methyl esters, or FAMEs (Sheehan, Dunahay, Benemann, \& Roessler, 1998). The principal reactant in the biodiesel conversion process is a short chain alcohol; typically methanol, $\mathrm{CH}_{3} \mathrm{OH}$. When methanol is deprotonated in solution and exposed to a triglyceride, it cleaves the glycerol group off of the triglyceride, resulting in one molecule of glycerol and three molecules of methylated fatty acids. This is known as a transesterification because the original organic functional group of the ester (glycerol) is exchanged for the organic functional group of a different alcohol (methanol). When methanol is used, a methyl group serves as the replacement alcohol during the reaction, and the more specific designation, transmethylation, is used to describe it. An illustrated description of the type of transesterification used in the current research is provided in the Materials and Methods section.

After transmethylation is carried out in a commercial biodiesel production process, the product must be isolated and purified. Glycerol is denser than biodiesel and can be drained out of a reactor (Gerpen, 2005). Impurities are removed by washing the product with water (Gerpen, 2005). Residual methanol is removed by distillation (Gerpen, 2005). 


\section{Lipid Quantification and Characterization}

The analytical methods of extraction, quantification, and characterization of lipids are more exacting than those used in an industrial setting, where incomplete reactions may be tolerated.

\section{Laboratory Transesterification}

The transesterification process for analytical samples is rigorous. A heated, basecatalyzed, transesterification reaction is often used in laboratory settings in order to maximize conversion (Karmee, Mahesh, Ravi, \& Chadha, 2004), (Tapanes, Aranda, Carneiro, \& Antunes, 2007). The method used in the current research will be described in greater detail in the Materials and Methods section of this document.

\section{Chromatographic Analysis}

Gas chromatography and mass spectrometry allow the determination of the molecular composition of volatile materials. These analyses can be performed directly in series on the same sample using a gas chromatograph coupled with a mass spectrometer, GC-MS.

By heating a sample, which has been injected into a long capillary column, the GC component separates constituents of the sample based on the rates at which they travel through the column. As compounds emerge from the end of the capillary column, their abundance is measured by a detector. The abundance of each constituent of the sample over time is expressed in a chromatogram plot. 
In the MS component of the instrument, eluting compounds are broken apart or ionized by a beam of electrons. Molecules which have taken on a charge, but not been broken apart, are called parent ions. The parent ions and fragments travel through a device called a quadrupole. In the quadrupole, fragments advance through a space surrounded by four parallel, electromagnetic rods. The variation of radio frequency voltage between each set of opposite rods destabilizes the trajectories of the fragments. For a given ratio of voltages, only fragments of a certain mass to charge, $\mathrm{m} / \mathrm{z}$, ratio will advance beyond the rods to a detector normal to their trajectories; all other fragments will be diverted into the rods. The abundance reported by the gas chromatograph component of the instrument is the number of parent ions which hit the detector per second. The output of a mass spectrometer is called a mass spectrum and it reports the mass to charge ratio of ionized eluting compounds on the horizontal axis and their abundance on the vertical axis.

A mass spectrum is useful for identifying compounds because it illustrates the relative abundance of functional groups in the compound. This allows a trained user to ascertain the composition of a compound. Modern mass spectrometers commonly incorporate software which allows mass spectra to be compared by a computer to a large database of known spectra via the internet. This facilitates rapid identification and also simplifies the process of determining the confidence with which a compound may be identified.

GC-MS analysis is a very powerful tool, but a great deal of care must be taken in order to ensure accurate results. Concentrated analytes are favorable because a large sample size increases the confidence of identification. However, analytes that are too concentrated can cause irreparable damage to fragile mass spectrometer components. In traditional 
GC-MS analysis, this problem requires analysts to perform analyses repeatedly until an analyte solution of ideal concentration is reached.

This problem can be avoided by operating a GC-MS in split mode. In split mode, the sample is injected into the septum and vaporized, just as it is in standard operation. In split mode, a fraction of the carrier gas-analyte mixture is diverted away from the GC column by a valve. The fraction diverted away from the column is wasted. This procedure has several purposes.

First, split mode operation is effectively a method of diluting the sample. Since mass spectrometers can be damaged beyond repair by a high rate of eluent impacts, the dilution of a sample can prevent damage to the instrument. Second, the use of split mode allows analysts to prepare an analyte solution that is more concentrated than would be possible using standard, non-split GC-MS operation. However, despite these benefits, split-mode injection also has some disadvantages.

As a sample stream is divided into two streams, it is critical to the accuracy of the analysis that portion that uniformly represents the sample is directed into the GC column. Biological samples typically contain a wide variety of molecular species and it has been observed in some studies that, owing to their relative lack of momentum, smaller molecules are more likely to be diverted to waste than larger molecules (Eder, Reichlmayr-Lais, \& Kirchgessner, 1991). This phenomenon is known as sample discrimination and a number of strategies have been successfully used to limit its effect on the quality of chromatographic analysis. 
A very high rate of sample vaporization helps to mitigate the effects of discrimination at the site of injection (Bannon, Craske, Felder, Garland, \& Norman, 1987). Rapid vaporization of the sample can be achieved if three criteria are sufficiently met. First, the sample must be injected rapidly. Repeatable high-speed injected can be done by a highly experienced analyst, but is more reliably achieved by an auto sampler. Second, the temperature of the septum must be sufficiently high compared the vaporization temperature of all of the compounds present in the analyte that the entire mixture changes phase quickly. Although a very hot injection site can damage certain analytes, it is ideal to use the highest temperature that does not damage any sample material. Third, a relatively dilute sample improves the uniformity with which the target compound is dissolved in the sample (Bannon, Craske, Felder, Garland, \& Norman, 1987).

Any compounds analyzed by GC-MS must be compared with a point of reference in order to produce quantitative data. A point of reference can come from a calibration curve or an internal standard and relates the abundance of the compounds in the sample to the abundance of a known concentration of a related compound (Volmer, Meiborg, \& Muskiet, 1988). There are advantages and disadvantages to each of these simple reference methods.

The construction of a calibration curve requires the preparation of multiple standard solutions containing different concentrations of compounds similar or identical to those compounds to be analyzed. The GC-MS results of an experimental sample are compared to the calibration curve to obtain concentrations of the target analytes. Although this method allows the use of the exact target analyte in the construction of the standard 
curve, it does not account for run-to-run variation in the analytes, particularly variation due to inconsistent injection technique (Volmer, Meiborg, \& Muskiet, 1988).

Alternatively, an internal standard is a compound similar, but not identical, to the target compound, which is added directly to the sample to be analyzed. The reported abundance of the internal standard is compared with those of compounds naturally present in the sample. The ratio of the total abundance of the standard to the known concentration of the standard is used to calculate the concentration of the target species. Although this method accounts for run-to-run variation, since it includes only one reference point, it does not account for changes in the ratio of abundance to concentration occurring over the time of elution (Volmer, Meiborg, \& Muskiet, 1988).

The bracket method of Volmer, Meiborg and Muskiet, (1988) has been demonstrated to be a reliable procedure for obtaining accurate analyte concentrations. It is a variation of the internal standard method. First, a sample of the analyte material is analyzed by the GC-MS. The first run is not quantitative, but is used to confirm that the compounds the analyst intends to use as internal standards are not naturally present in the sample. After determining which compounds do not exist in the sample, the analyst selects two standards which are closely related to the analytes. One standard should have a boiling point directly below the constituents of interest, and the other standard should have a boiling point directly above the compound of interest. The average recovery of the two standards is used to determine the concentration of the analyte materials. It has been demonstrated that for the analysis of fatty acid methyl esters, the bracket method results in consistently lower coefficients of variation than the use of a single internal standard (Volmer, Meiborg, \& Muskiet, 1988). 


\section{Chapter 3: Materials and Methods}

The first sections of this chapter describe the cultivation of microalgae used in the lipid quantification and characterization studies. The latter sections provide details of the methods used for the microalgae analyses. Throughout the Materials and Methods section, laboratory equipment is discussed in brief. Comprehensive information on the equipment used is provided in the section entitled Appendix D: Equipment List.

\section{Setup and Operation of the Algae Pond}

Microalgae were cultivated in an open pond to grow biomass for lipid analysis. The pond was operated in batch mode to simplify the observation of time-dependent changes in the microalgae culture. The algae used to inoculate the experiments were grown in an outdoor, continuous-flow pilot algae treatment system at the San Luis Obispo Water Reclamation Facility. The pilot system, operated by Cal Poly graduate student Michael Podevin, used small paddle wheel-mixed high rate ponds fed primary clarifier effluent. The hydraulic residence time of the ponds was maintained at 5 days.

\section{Pond Configuration}

The batch-growth pond used in the present research was a 2195 liter rectangular fiberglass tub with 0.75 meter tall sidewalls (Figure 4). While operating, the pond was filled to a depth of approximately $20 \mathrm{~cm}$. In the center of the pond, a plastic partition was erected to recreate the hydraulic configuration of a raceway. A four-bladed paddle wheel, rotating at eight cycles per minute, was used to provide circulation of the water. 
The pond was sparged with pure carbon dioxide in order to prevent limitation of algal growth by low inorganic carbon concentration. This technique is described in a previous thesis project at Cal Poly (Feffer, 2007). The flow of carbon dioxide was adjusted each day in pursuit of a target $\mathrm{pH}$ of approximately 7.75 , a favorable condition for algal growth.

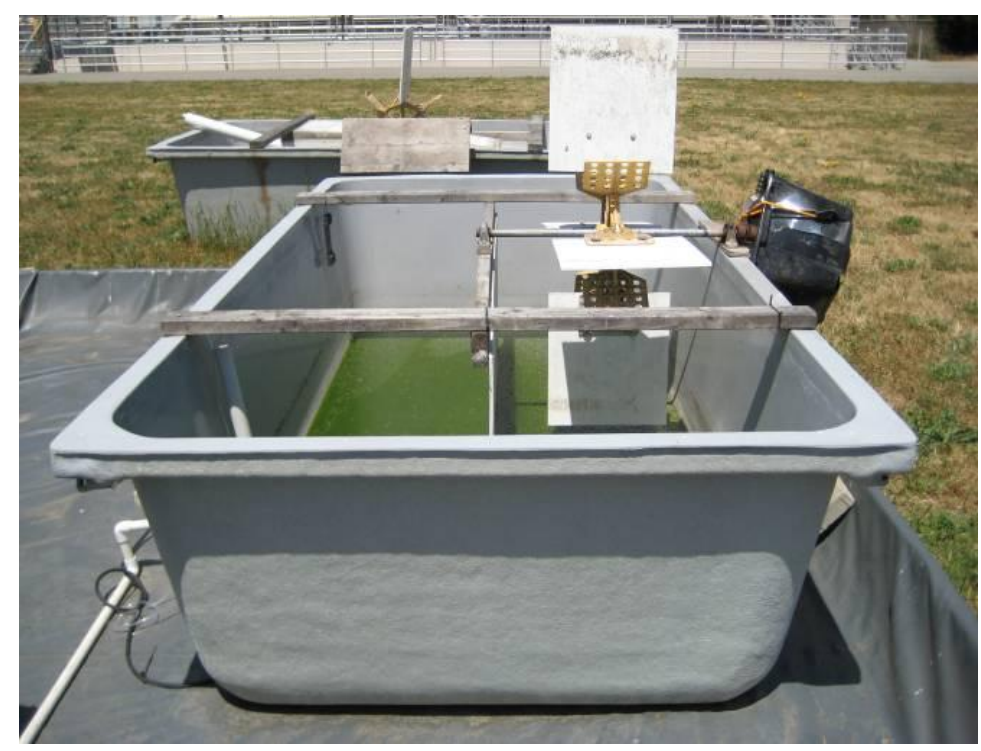

Figure 4: Batch-mode pond setup

The algae pond used for this experiment was operated in batch mode to facilitate observation of lipid concentration and characteristics at progressive culture ages and nutrient depletion levels. Batch growth also provided a dense culture, which allowed a higher sensitivity in the resulting lipid data. 


\section{Inoculum and Growth Medium}

The pond was filled with $855 \mathrm{~L}$ of effluent from the primary clarifier of the 4.5 MGD San Luis Obispo Water Reclamation Facility. The wastewater used in the pond was collected between 2:00 p.m. and 5:00 p.m., at the beginning of the evening peak of sewage flow. The effluent contained $80 \mathrm{mg} / \mathrm{L}$ of total suspended solids and $72 \mathrm{mg} / \mathrm{L}$ of volatile suspended solids. Analyses conducted by Cal Poly graduate student Michael Podevin throughout March and April suggest that the 5-day biological oxygen demand of the effluent was approximately $175 \mathrm{mg} / \mathrm{L}$.

The pond was inoculated with $95 \mathrm{~L}$ of water from an adjacent continuous-mode algae pond fed the same primary effluent. The continuous pond was constructed similarly to the batch-mode pond, but it was not sparged with carbon dioxide. The inoculum contained $212 \mathrm{mg} / \mathrm{L}$ of total suspended solids and $175 \mathrm{mg} / \mathrm{L}$ of volatile suspended solids. Based on analyses performed by Michael Podevin, it is estimated that the 5-day biological oxygen demand of the inoculum was approximately $25 \mathrm{mg} / \mathrm{L}$.

The purpose of inoculating the batch-mode pond with water from a nearby continuous pond was to simulate conditions which may be achievable in a full-scale algae system. Since the microalgae harvested from the continuous pond had been grown outdoors on municipal wastewater, their ability to survive on municipal wastewater in outdoor conditions had been demonstrated prior to the initiation of the batch experiment. 


\section{Pond Operation}

The pond was inoculated on March 23, 2009 and operated for 25 days through April 17. Once inoculated, the management of pond consisted only of daily adjustments to the carbon dioxide flow to maintain a slightly alkaline $\mathrm{pH}(\sim 7.75)$. The air temperature fluctuated between a high of $26^{\circ} \mathrm{C}$ on both March 28 and April 5 and a low of $9{ }^{\circ} \mathrm{C}$ on April 15. A rainfall event took place over a period of two days between April 7 and 8, with a total precipitation of $<1 \mathrm{~mm}$. Weather data were gathered from the National Oceanic and Atmospheric Administration, NOAA, database (NOAA National Climatic Data Center, 2009). The NOAA data were obtained from a weather station at the San Luis Obispo County Regional Airport, approximately three kilometers from the algae pond. High and low daily air temperatures from the weather station are shown in the Figure 5. The dates over which the two day-long rain event occurred are indicated by a single cloud.

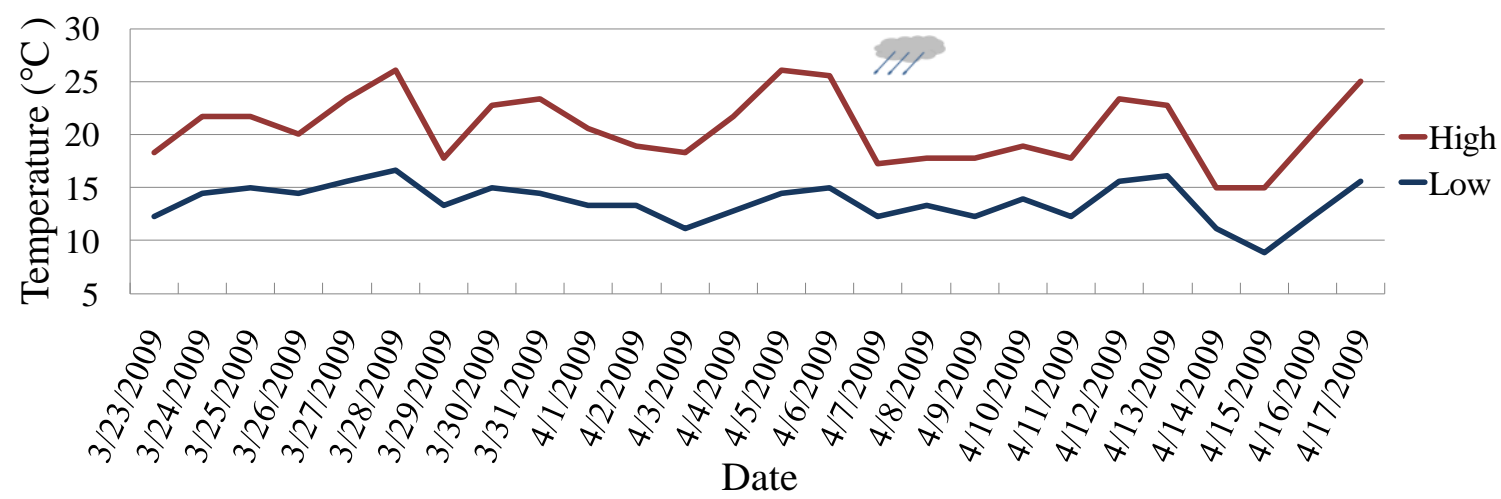

Figure 5: Weather throughout pond experiment 


\section{Sample Collection}

Samples were collected from the pond daily, typically between noon and 2:00 p.m. The volume collected depended on which analyses were to be performed each day. After removal from the pond, samples were rushed within 15 minutes to a Cal Poly laboratory where their headspace was purged with nitrogen and they were placed in a refrigerator. Samples were kept in the refrigerator for no longer than three hours before analysis was initiated or steps were taken for longer-term preservation.

Long-term preservation involved centrifuging the samples to remove most of the water and freezing under an $\mathrm{N}_{2}$ atmosphere. This procedure will be discussed in greater detail in the section entitled Bligh and Dyer.

\section{Experimentation}

Two sets of experiments have been conducted over the course of this research. One study involved daily analysis of the lipids in the batch-mode pond. This study was meant to provide information about the development of lipids over the growth and death of an algal culture.

The other study involved the comparison of a number of different procedures for the extraction of the lipids from the algae grown in the pond. 


\section{Lipid Production Experiment}

A series of analyses were conducted to relate the growth stage of the batch-mode algal culture to the quantities and types of lipids present in the algal mass. The study involved regular testing of total and volatile suspended solids of the pond, as well as determination of the lipid content of the algae in the pond. The sampling scheme is described below. The primary effluent used to fill the pond and the algae used to inoculate the pond were tested in the same manner, prior to the initiation batch growth. On Day 11 of the experiment, the total and volatile suspended solids concentrations of the supernatant fluid were tested after the centrifugation of the sample. 


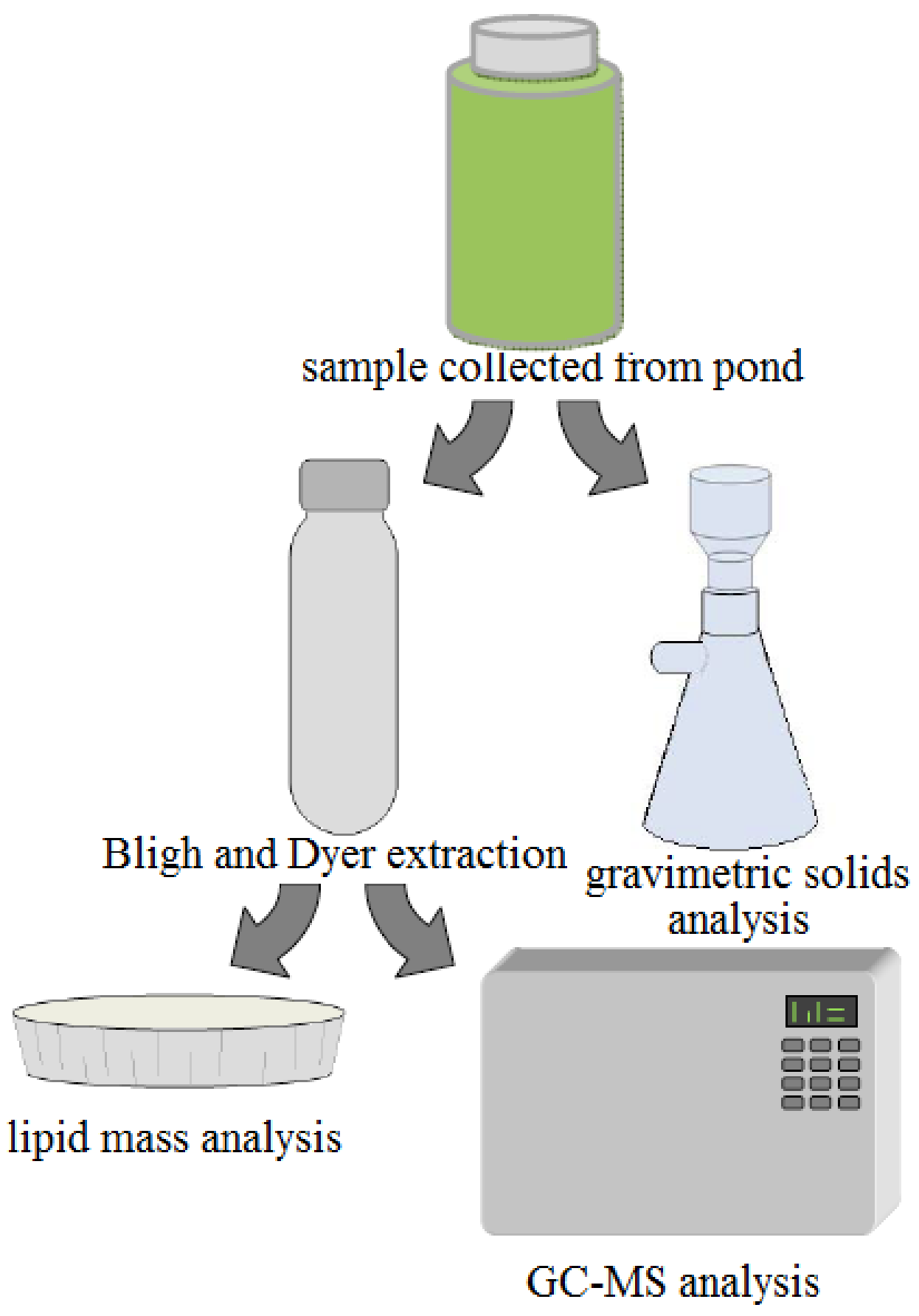

Figure 6: Lipid production experiment sampling scheme 


\section{Solids Determination}

Tests for total and volatile suspended solids were conducted to estimate the concentration

of algae in the pond. Solids tests were conducted according to Standard Methods 2540 D (Greenberg, 1995).

First, a number of $47 \mathrm{~mm}$-diameter, 1.2-micron Fisher G4 glass fiber filters were rinsed with deionized water and dried in a $105^{\circ} \mathrm{C}$ oven to remove any extraneous material from the manufacturing process. The filters were rinsed with deionized water, one at a time, using a vacuum filter apparatus. One filter was prepared for each sample to be analyzed. The filters were stacked in an aluminum weighing dish with one extra filter on top of the stack and one extra filter on the bottom. The purpose of the extra filters was to prevent the accumulation of any ash on the filters which were to be used for analysis. The weigh boat containing the filter stack of filters was placed in a $525^{\circ} \mathrm{C}$ muffle furnace for fifteen minutes.

After removal from the furnace, the filters were cooled in an approximately $20^{\circ} \mathrm{C}$ desiccator cabinet before each was placed in a separate labeled aluminum dish and weighed to the nearest $0.01 \mathrm{mg}$.

A $20 \mathrm{~mL}$ aliquot of the sample water was filtered through each filter using the vacuum apparatus. The filters were then replaced in their aluminum dish and placed in a $105{ }^{\circ} \mathrm{C}$ oven for two hours. The purpose of heating was to isolate dry biomass on the filter by vaporizing all of the water from the sample. 
After two hours, the dishes were removed from the oven and allowed to cool to room temperature in a desiccator cabinet before they were weighed to the nearest $0.01 \mathrm{mg}$. This weight was used to determine the total concentration of solids, or TSS, in the original sample, according to the following equation.

$$
T S S=\frac{105^{\circ} \mathrm{C} \text { Mass }- \text { Tare }}{\text { Volume Filtered }}
$$

The trays were then placed in a $525^{\circ} \mathrm{C}$ muffle furnace for fifteen minutes. After the muffle furnace, they were placed in a desiccator to cool to room temperature and then weighed to the nearest $0.01 \mathrm{mg}$. The weight was used to determine the concentration of volatile suspended solids in the original sample, according to the following equation:

$$
\text { VSS }=\frac{105^{\circ} \mathrm{C} \text { Mass }-525^{\circ} \mathrm{C} \text { Mass }}{\text { Volume Filtered }}
$$

The concentration of volatile suspended solids was used to approximate the concentration of algal biomass in the water. All total and volatile suspended solids tests were performed in triplicate.

\section{Microscopic Investigation}

Over the course of the operation of the batch-mode pond, a microscopic analysis of the micro biota present was performed every five days. The inoculum and growth medium were also examined using a microscope. An Olympus CX 41 microscope was used in conjunction with Olympus software to capture images of the algae growing in the culture. A hemacytometer was used prior to each microscope session to calibrate the software and 
provide an accurate scale-bar in the images. Tentative identifications to the genus level were made using a freshwater algae key (Prescott, Bamrick, Cawley, \& Jaques, 1978).

\section{Lipid Investigation}

Throughout the operation of the batch-mode pond, analyses were conducted of the quantity and the identity of lipids growing in the algae. The same extraction procedure was used in both cases.

Bligh and Dyer Extraction

The Enssani (1987) adaptation of the Bligh and Dyer procedure was used to extract lipids from the algae for further study.

For each test to be performed, a $200 \mathrm{~mL}$ aliquot of water collected from the pond was centrifuged into a pellet at approximately $2800 \mathrm{~g}$. The relative centrifugal field was determined as follows:

$$
\text { Relative centrifugal field }=\frac{\omega^{2} r}{g}
$$

where $\omega$ is the angular velocity in radians per second, based on a rotational frequency of 4,000 rotations per minute. Although the centrifuge used was throttled by a powerstat and did not report rotations per minute, the rotational speed was assumed from similar models. The value $r$ is the distance between the center of the centrifuge tube and the center of rotation, $8.8 \mathrm{~cm}$, and $\mathrm{g}$ is the acceleration of gravity. 
Due to the limitations of test tube capacity, the centrifugation process was undertaken 40 $\mathrm{mL}$ at a time. After $40 \mathrm{~mL}$ had been centrifuged for four minutes, the supernatant liquid was poured off from each sample and replaced with $40 \mathrm{~mL}$ of sample water. This process was repeated until the solids from $200 \mathrm{~mL}$ of sample water had been collected in a pellet at the bottom of each test tube.

Centrifugation was performed in tared, solvent resistant, $50 \mathrm{~mL}$, Teflon, round bottom centrifuge tubes. A room temperature table-top centrifuge was used. The centrifugation of each pellet required approximately 25 minutes and the pellets were centrifuged four at a time.

After the samples had been pelleted in the centrifuge tubes, they were either used for extraction immediately or were flushed with nitrogen and placed in a freezer until needed.

The first step of the extraction was to add $5 \mathrm{~mL}$ of chloroform, $10 \mathrm{~mL}$ of methanol and 4 $\mathrm{mL}$ of deionized water to each tube containing a pelleted sample of algae. The methanol, which is miscible in the water layer, is included to amend the polarity of the water layer to limit the concentration of polar lipids such as chlorophyll and phospholipids in the chloroform layer. Once the tubes had all reached room temperature, a sonicator was used to disrupt the algae cells in the suspended mixture. A Branson Ultrasonics sonicator was operated at a constant duty cycle on power level 8 . Each tube was sonicated for one minute. The sonicator tip was rinsed between each sonication to remove any residual material. The samples were then placed horizontally on a shake table with a single-axis motion of $6 \mathrm{~cm}$ oscillations at 2 cycles per second for between 6 and 8 hours. The 
purpose of the shaking step was to promote the complete exposure of intracellular products to the solvents.

The tubes were removed from the shake table and an additional $5 \mathrm{~mL}$ of chloroform and $5 \mathrm{~mL}$ of deionized water were added to each sample. Each tube was vortex mixed for 30 seconds to mix the newly added solvents. The tubes were then centrifuged at $4850 \mathrm{~g}$ for four minutes to separate the contents into layers.

A test tube containing the mixture at this stage is shown in Figure 7.

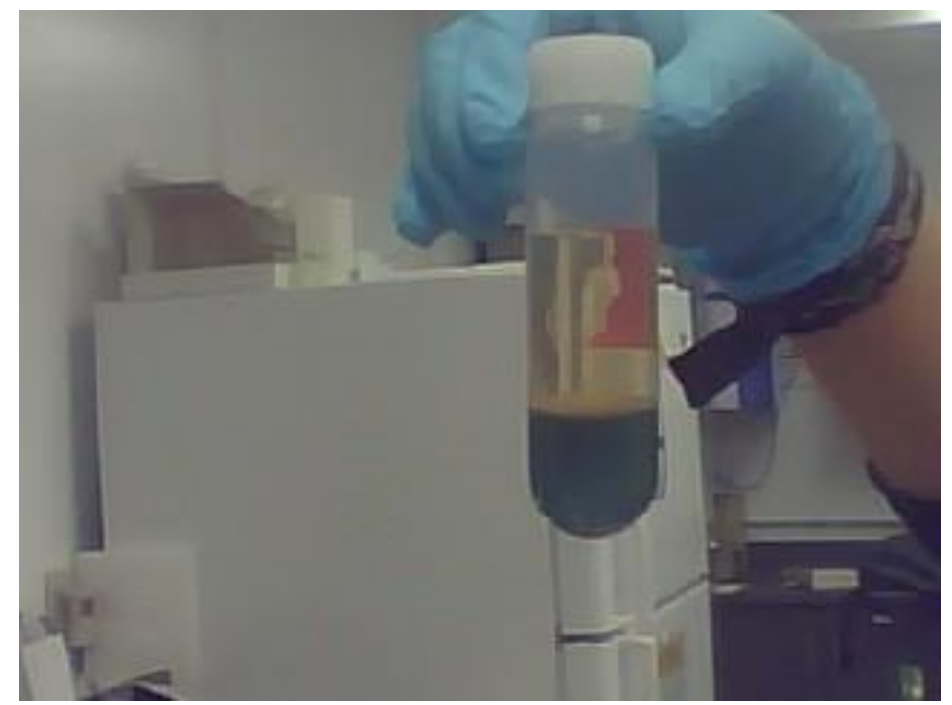

Figure 7: Bligh and Dyer extraction with chloroform layer at bottom

The green layer at the bottom, comprised primarily of chloroform, contains lipophilic material. The upper layer contains methanol and water. A thin layer of cell debris separates the two layers. 
The lower, lipid-rich chloroform layer was removed using a glass Pasteur pipette for additional testing. This extract was pipetted into a $60 \mathrm{~mL}$ Luer-lock syringe made of solvent-resistant polypropylene. The syringe was used to force the extract through a 0.2 $\mu \mathrm{m}$, nylon syringe-driven filter. Depending on whether the extract was to be used for lipid quantity analysis or lipid identification, it was injected into either a tared aluminum weighing dish (4 $\mathrm{cm}$ diameter) or a tared glass test tube $(50 \mathrm{~mL})$.

After the extract had been pipetted and filtered, an additional $10 \mathrm{~mL}$ of chloroform were added to each of the Teflon centrifuge tubes. The tubes were again vortex mixed for 30 seconds each and centrifuged at $4850 \mathrm{~g}$ for four minutes. The chloroform layer was again pipetted from the bottom of each tube into the same vessel as had been used during the prior set of extractions.

\section{Lipid Mass Determination}

For the preparation of samples to be used for the determination of lipid mass, a syringedriven filter was used, as described previously, to deposit the algae extract into a tared aluminum weighing dish. The use of aluminum trays, as opposed to glass test tubes, is preferable for the weight determination of lipids because the trays are not as massive compared with the lipids as test tubes would be. This helps to minimize balance error.

After the second extraction had been performed, the weighing dishes were placed in an approximately $20^{\circ} \mathrm{C}$ desiccator cabinet which was supplied with a constant flow of nitrogen. The flow of nitrogen through the desiccator was meant to limit oxidation of the lipids that could have taken place while the extracts were drying. The extracts remained 
in the desiccator until no chloroform was visually apparent in the dishes (typically 24 hours).

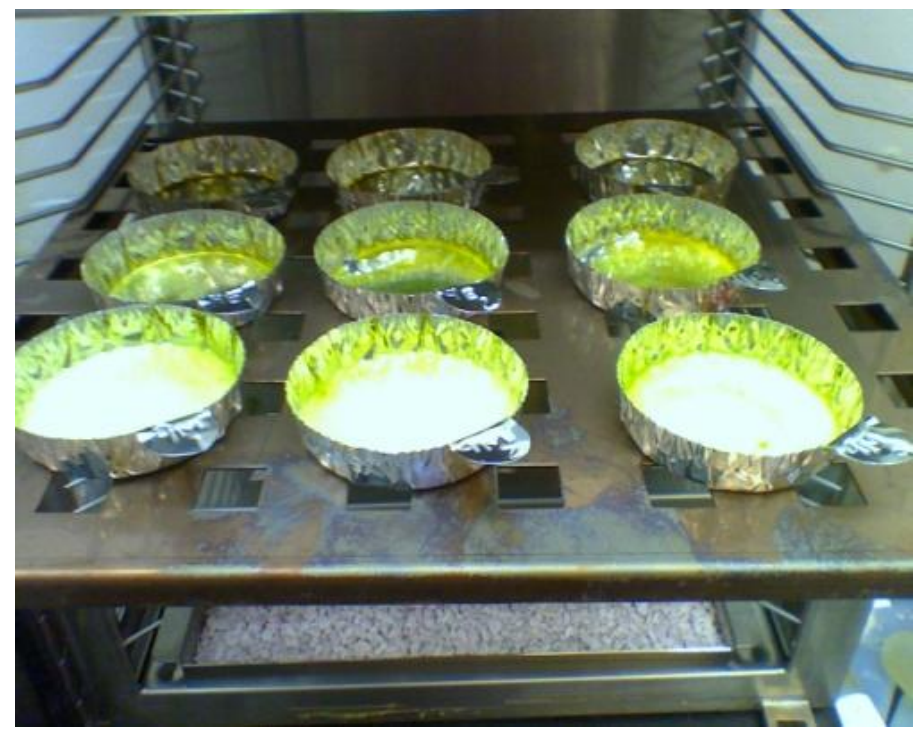

Figure 8: Samples in the nitrogen-sparged desiccator

The weighing dishes were then placed in a $105^{\circ} \mathrm{C}$ oven, which was also flushed continuously with nitrogen, for one hour. The purpose of this step was to ensure that all of the solvent had been removed from the weighing dishes, leaving only algal lipids.

After drying in the oven, the dishes were allowed to cool in a nitrogen-sparged desiccator and weighed to the nearest $0.01 \mathrm{mg}$. The weight measurements were used to determine the concentration of lipids in the algae and were used with total volatile solids data to calculate the concentration of lipids in the algae water. The mass of each sample was measured in triplicate. 
Although the Bligh and Dyer procedure is a common laboratory method, it is suspected of overestimating the lipid mass of microalgae (Woertz, Feffer, Lundquist, \& Nelson, 2009), (DeLuca, Charity. pers. comm., 2009). The solvent system extracts nonpolar nonlipid material in addition to triglycerides, which can cause an overestimate of total lipid mass. The Bligh and Dyer method is not selective enough to exclude lipophilic compounds such as chlorophyll from extraction (Woertz, Feffer, Lundquist, \& Nelson, 2009). This error is not a severe problem when oilseeds are the subject of research, as they do not contain chlorophyll (Lehr, Corinne. pers. comm., 2009).

\section{Triglyceride Identification}

In addition to mass analysis, samples from the pond were extracted each day to identify the types of triglycerides that they contained. The Bligh and Dyer procedure was used, exactly as in the samples used for weight determination. However, instead of extraction into weighing dishes, the samples used for lipid analysis were extracted into glass test tubes. Glass test tubes are preferable to weighing dishes for this analysis because they are sealable. A spill-proof seal facilitates the mixing of toluene with the lipids, which is necessary for chromatographic analysis.

The extracts in the test tubes were dried to constant weight in a Caliper Life Sciences nitrogen-sparged desiccator apparatus at $30{ }^{\circ} \mathrm{C}$, which typically required 4 hours. It was not possible to use the same apparatus with the lipid mass determination samples because aluminum weighing dishes do not fit in the Caliper Life Sciences desiccator. Had the weighing dishes and desiccator been compatible, the lipid mass determination samples would have been dried in the Caliper Life Sciences system, as well. 
Transmethylation

After extraction into glass tubes, the extracts were either used immediately for lipid analysis, or the headspaces were flushed with nitrogen and the tubes were placed in a freezer.

Because triglycerides are relatively large molecules and have high boiling points, they are not easily analyzed by gas chromatography. In preparation for chromatographic analysis, the lipid samples were transmethylated in order to cleave each triglyceride into its constituent fatty acids. This step makes possible the chromatrographic processing needed to identify triglycerides. The transmethylation reaction resulted in the production of fatty acid, methyl esters, which, incidentally, are the principle molecules of biodiesel fuel.

The first step of the transesterification was to resuspend up to $10 \mathrm{mg}$ of the oil extracts in $1 \mathrm{~mL}$ of dry toluene. Since different tubes contained different masses of lipid material, the volume of toluene added varied between samples. In each case, enough toluene was added so that no more than $10 \mathrm{mg}$ of lipids were present in the tube for each milliliter of toluene. A $1 \mathrm{~mL}$ aliquot of the dissolved solution was then pipetted into a new tube for further processing.

Anhydrous sodium methoxide in methanol ( $2 \mathrm{~mL}$ of $0.5 \mathrm{M}$ solution) was added to each sample. This basic reagent was selected because the continual regeneration of methoxide reactants during the transesterification promoted a complete reaction. In the basecatalyzed reaction, as fatty acids are cleaved from triglycerides, they form a highly basic glyceroxide which can acquire a proton from methanol, regenerating the methoxide reagent. The reaction mechanism is illustrated Figure 9. 


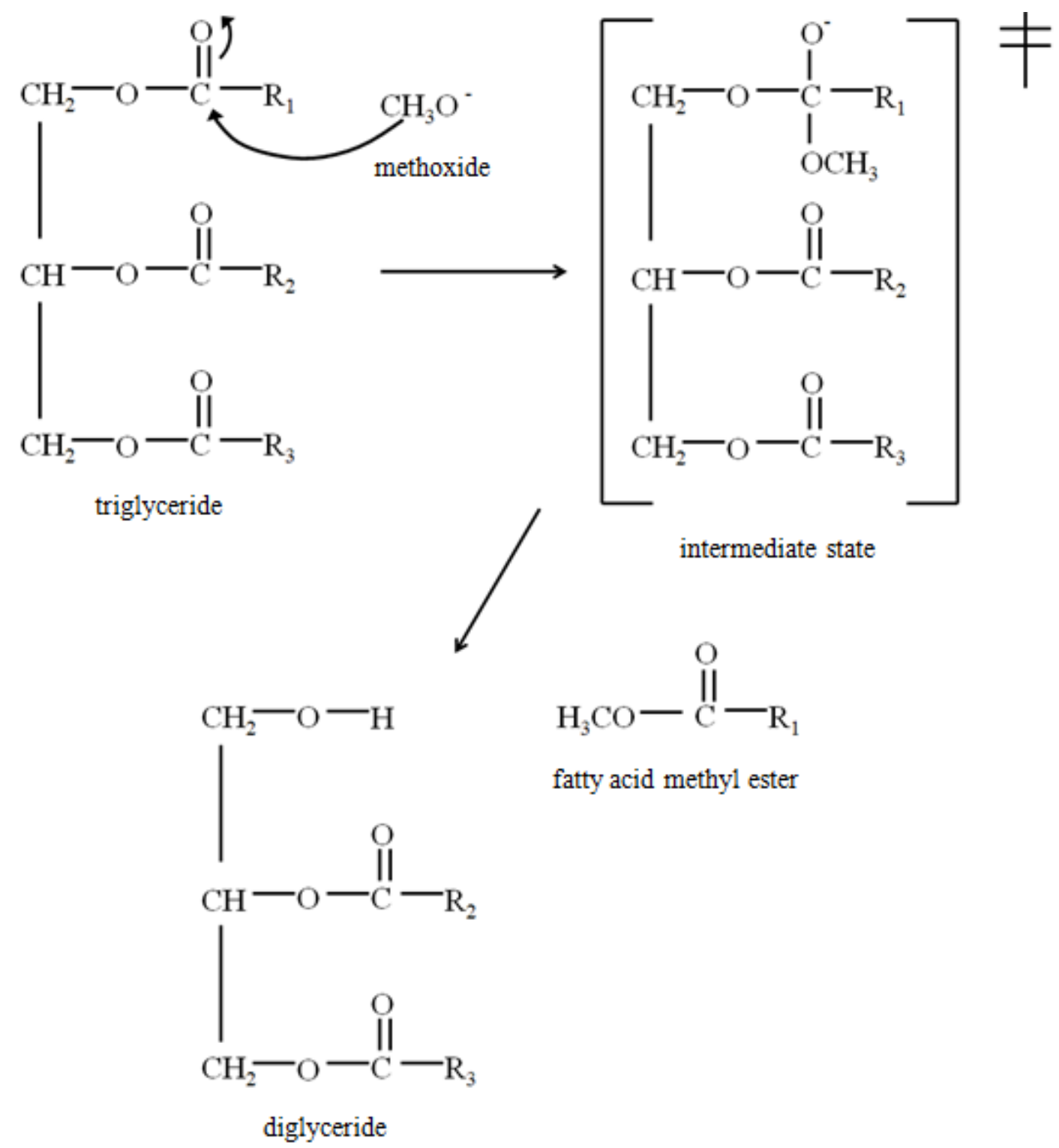

Figure 9: Base-catalyzed reaction mechanism

After the addition of the sodium methoxide solution, the reaction was allowed to proceed for ten minutes in a $50{ }^{\circ} \mathrm{C}$ water bath. After the mixture had reacted for ten minutes, the methoxide was neutralized by the addition of $0.1 \mathrm{~mL}$ of glacial acetic acid to each 
sample. A $5 \mathrm{~mL}$ aliquot of hexane and a $5 \mathrm{~mL}$ aliquot of deionized water were added to each sample. Each tube was then shaken by hand for approximately 30 seconds.

After the mixed samples had been allowed to settle for approximately five minutes, the FAME-rich hexane layers were each transferred by glass pipette to a clean glass test tube. An additional $5 \mathrm{~mL}$ of hexane were added to each tube and the mixtures were again shaken. After separating into layers, the hexane layer from each sample was pipetted into the glass test tube which already contained the first extract from it.

Anhydrous sodium sulfate was added to each tube of extract. The extracts were shaken for approximately 15 seconds to promote removal of residual water by the sodium sulfate. Each tube of extract was then gravity filtered through Fisher P5 cellulose paper into a glass test tube to remove the sodium sulfate. If the samples were not to be used immediately, the headspaces were flushed in nitrogen and the tubes were frozen.

\section{Gas Chromatography-Mass Spectroscopy}

After transmethylation, the samples were analyzed using a gas chromatograph-mass spectrometer. A small aliquot of each sample was diluted 1:10 in hexane in order to ensure that no constituent was present in such large concentration that a stronger dilution would damage the mass spectrometer.

An Agilent 6890 gas chromatograph was used with an Agilent 575S mass spectrometer for the testing. A fused silica $50 \mathrm{~m}$ by $0.25 \mathrm{~mm}$ column (Agilent \#190915-433) was used in the chromatograph. 
The chromatograph was programmed to run a 10:1 split with a sample size of $5 \mu \mathrm{L}$ for a total column flow of $13.7 \mathrm{~mL}$ per minute over the duration of the 10.20 minute run. The inlet was heated to $250{ }^{\circ} \mathrm{C}$ and the initial temperature of the column was $120^{\circ} \mathrm{C}$. After injection, the chromatograph ramped at $50{ }^{\circ} \mathrm{C}$ per minute to $280^{\circ} \mathrm{C}$ and held for an additional two minutes until the end of the run.

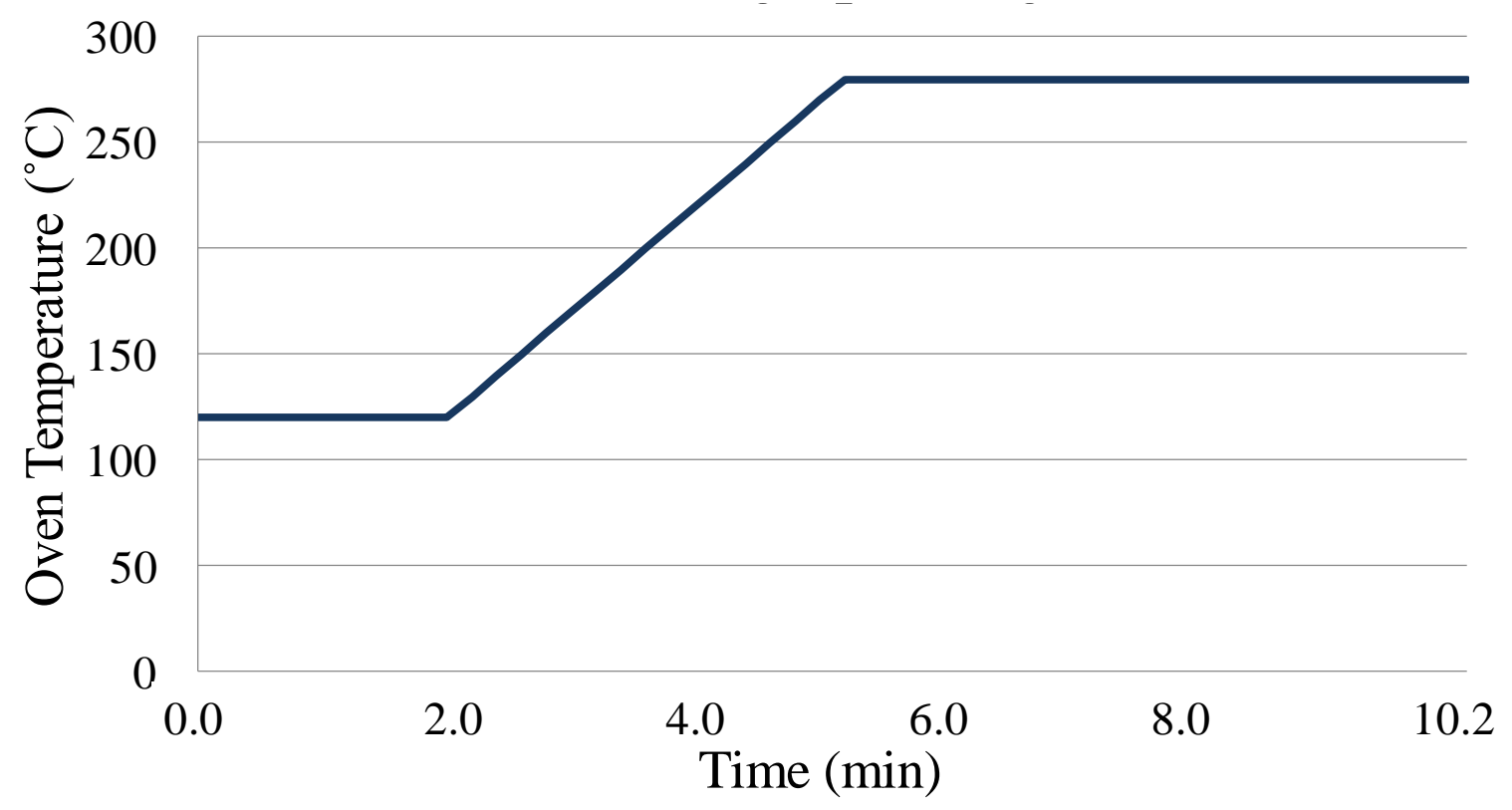

Figure 10: Gas chromatograph program

The quadrupole was heated to $250{ }^{\circ} \mathrm{C}$ and the mass spectrometer source to $230{ }^{\circ} \mathrm{C}$. An autosampler was used. The autosampler was programmed to flush twice with hexane prior to injection. It then rinsed with the sample three times and drew the final sample, using four fill-discharge cycles to minimize air bubbles in the syringe. Subsequent to injection, the autosampler rinsed twice with hexane. Each sample vial was analyzed by GC-MS in triplicate. 
The bracket method of Volmer, Meiborg and Muskiet was used to quantify the concentrations of each of the constituents of the lipid extracts. Pentadecanoic acid, methyl ester and heptadecanoic acid, methyl ester were selected as standards. These standards were chosen for three reasons.

First, since they are very closely related to the target compounds, they are expected to behave similarly during chromatographic analysis.

Second, despite a close structural relation to algae oils, they are not naturally present in algae samples. Since de novo synthesis of fatty acids is primarily conducted by the repeated addition of two-carbon malonyl-coA units to an existing carbon chain, fatty acids typically have an even number of carbon atoms in their primary carbon chains.

Third, these standards were selected because preliminary study had suggested that the fatty acid methyl esters derived from wastewater would elute after pentadecanoic acid, methyl ester and before heptadecanoic acid methyl ester.

After adding the standards to the samples, the concentration of each constituent of the samples was computed as follows. A proportionality factor was calculated for each standard by dividing the concentration of the standard by the area reported by the mass spectrometer. A unique constant was determined for each constituent of each standard. The rate of change of the proportionality constant over elution time was computed for the period of time between the elution of pentadecanoic acid methyl ester and nonadecanoic acid methyl ester. The rate of change was used to extrapolate a proportionality constant for each constituent in each sample. This proportionality constant and the peak area of the constituent of interest were used to determine the concentration of each constituent. 


$$
\begin{aligned}
& \text { area }=\int_{\text {Time } 1}^{\text {Time } 2} \text { Abundance } \\
& \text { Proportionality constant, } P=\frac{\text { Conc. }}{\text { Area }} \\
& \text { Slope }=\frac{\frac{\text { Conc }_{\text {C19 }}}{\text { Area }_{C 19}}-\frac{\text { Conc }_{\text {C15 }}}{\text { Area }_{C 15}}}{\text { Time }_{C 19}-\text { Time }_{C 15}} \\
& \text { Conc. } A=\text { Area } A \times P_{15}+\left[\text { Slope } \times\left(\text { Time }_{A}-\text { Time }_{15}\right)\right]
\end{aligned}
$$

A careful inspection was made of each peak with an area $2 \%$ or greater than the size of the largest peak in each chromatograph. Constituents present in very small quantities were omitted from the analysis, as they are difficult to identify with confidence.

Molecules were identified based on the time at which they eluted from the gas chromatograph and from the constituent peaks of their mass spectra. Mass spectra were compared with standards from the MS Search 2.0 database maintained by the United States National Institute of Standards and Technology (NIST) (National Institute of Standards and Technology, 2005). In approximately $10 \%$ of the samples, the mass spectra of some of the peaks did not align well enough with NIST standards to make a conclusive judgment of their identity. In these cases, the constituents were omitted from the results. These omissions did have a large impact on the results of the experiments because these peaks were identified conclusively in other samples.

Approximately 5\% of the samples contained contaminants which coeluted with important analytes. This phenomenon is often readily apparent by the occurrence of a shoulder in a chromatogram. A shoulder is a term given to a pair of peaks that are partially 
superimposed on a chromatogram. This makes it difficult to determine the true abundance of either constituent. Thus, coeluting compounds were omitted from the results. These omissions did not have a large impact on the results of the experiments because these peaks were identified conclusively in other samples.

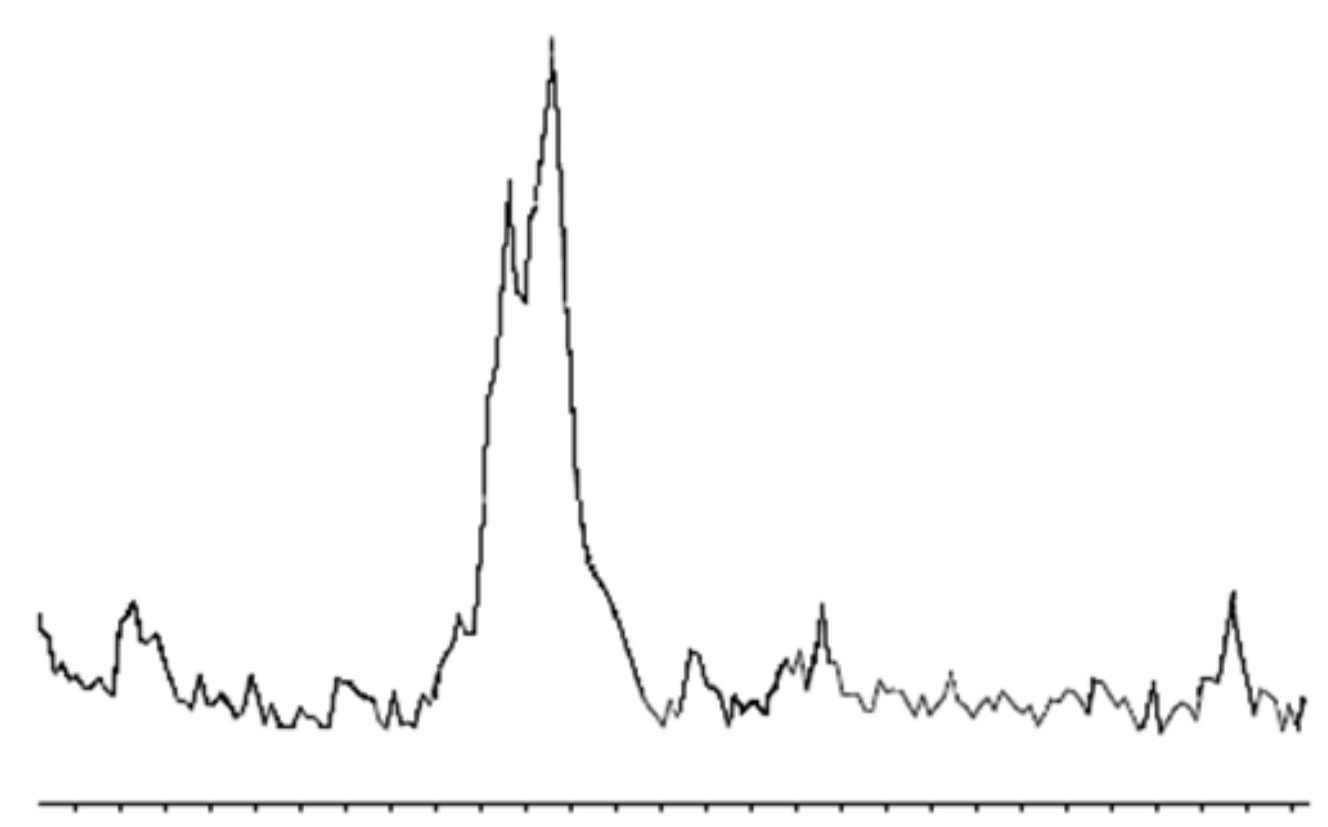

Figure 11: Shoulder feature in a chromatogram from Day 8

Several head-to-tail comparisons of sample mass spectra against NIST standards are shown below as examples. These comparisons were selected because they were readily identifiable. The spectrum on the upper portion of each graph is from samples taken during the current research. The spectrum on the lower portion of each graph is from the NIST reference database. 

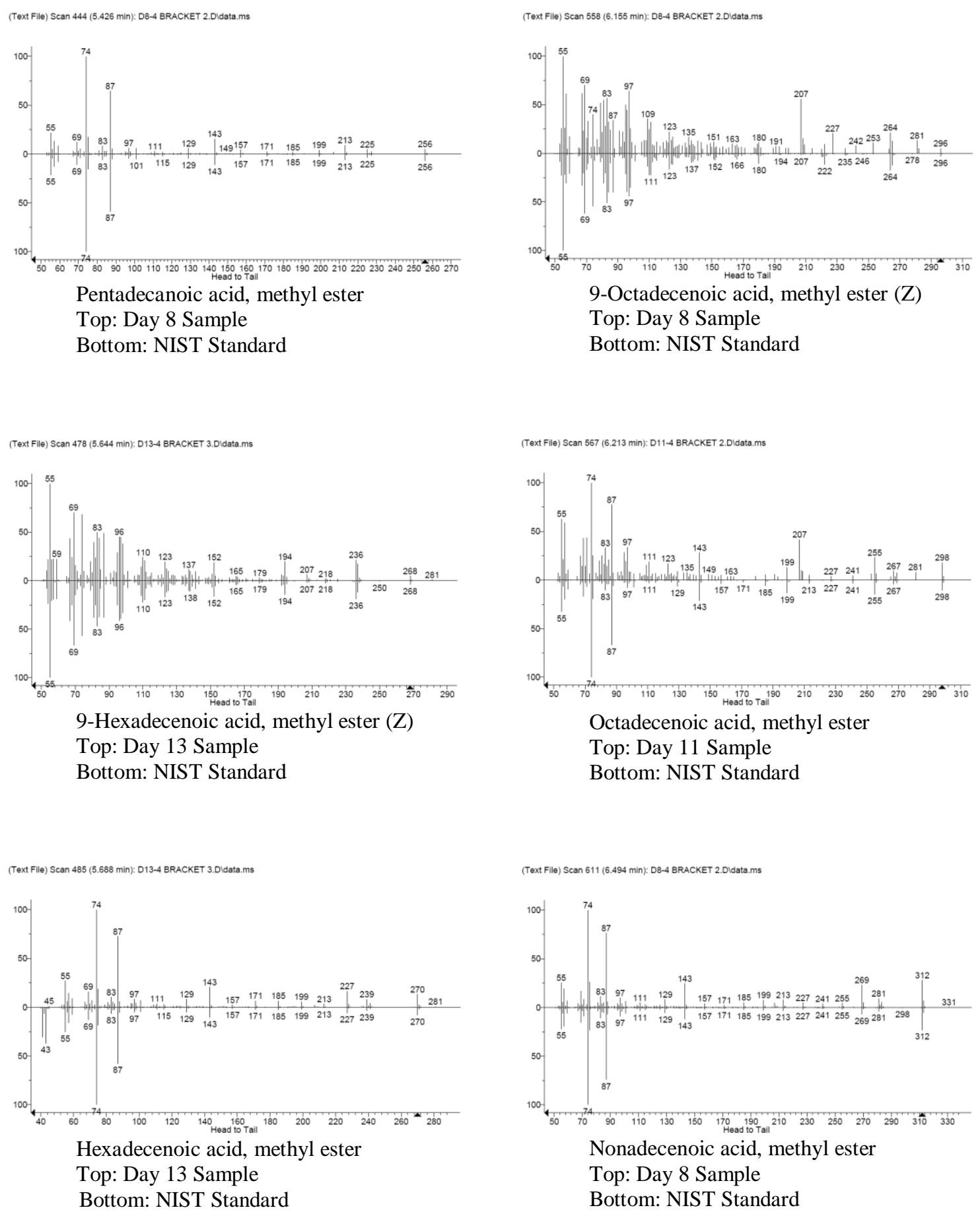

Figure 12: Head-to-tail comparisons of common constituents to reference chromatograms 


\section{Extraction Procedure Experiment}

An experiment was conducted to compare several methods of solvent extraction in terms of the quantity and types of lipids they extracted from algae. A large number of sample pellets were prepared on Day 9 of the operation of the batch mode pond. The samples were collected and stored as in the lipid production experiment. These samples were used to compare three experimental extraction procedures to the lab-standard Bligh and Dyer. Each of the three experimental procedures had identical steps, but used different counter-solvents: methanol, ethanol and isopropanol.

After the procedure, the extracts were evaluated for the quantity and identity of the lipids they contained. The analysis for both lipid mass and lipid characterization were performed by the same methods as in the lipid production experiment. The experimental scheme is illustrated in Figure 13. 


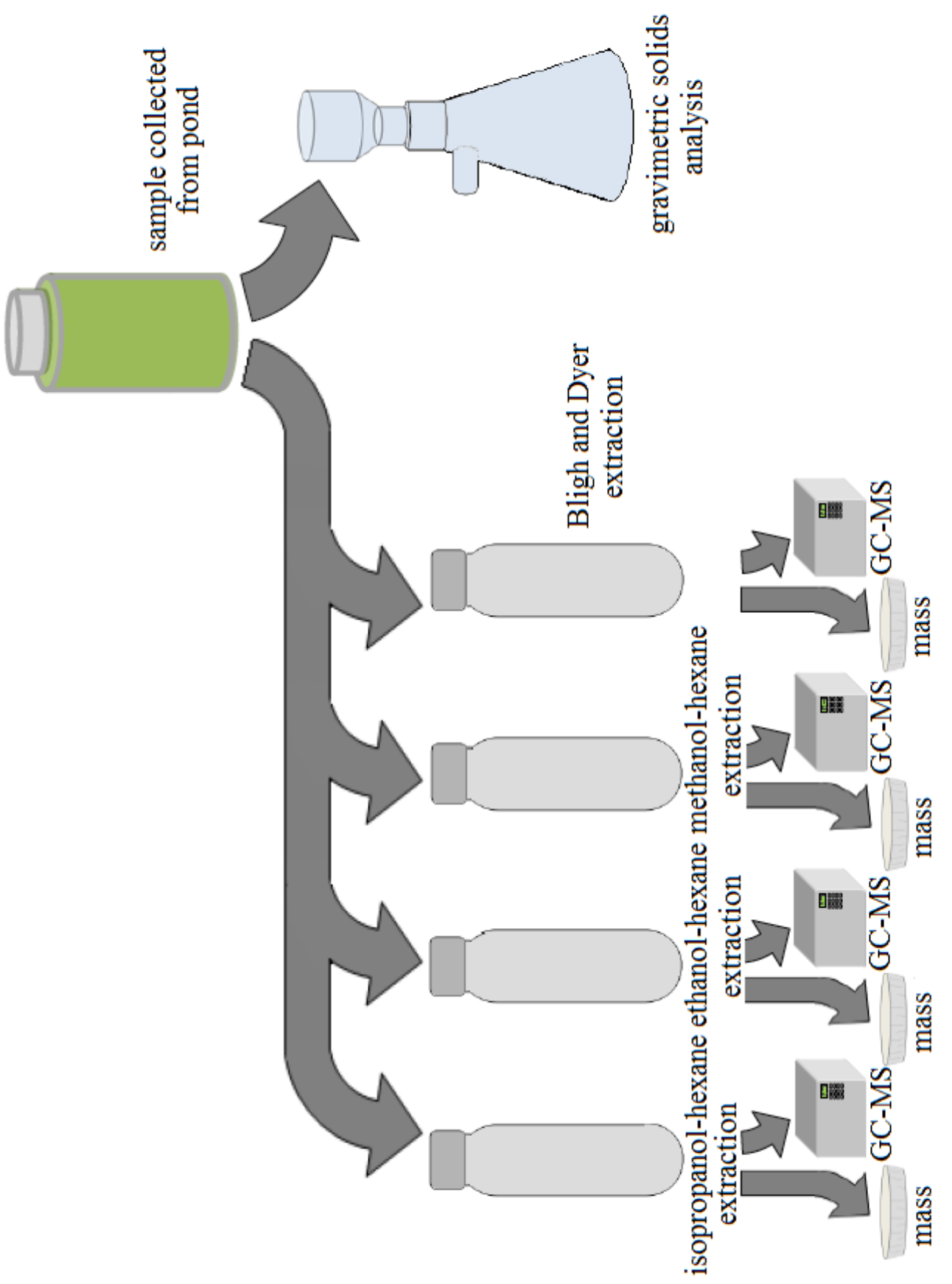

Figure 13: Extraction experiment scheme 


\section{Alcohol Extraction}

A simpler procedure was used to compare the different solvents against the Bligh and Dyer extraction. The purpose of the simple procedure was to simulate an extraction which may be possible on an industrial scale. The extractions used an alcohol-waterhexane solvent system. Methanol, ethanol and isopropanol were each tested as countersolvents. The electric dipole moments of each of these solvents are presented in Table 2.

Table 2: Dipole moments of organic solvents (Newton, 2009)

\begin{tabular}{|l||c|c|}
\hline & & Electric Dipole \\
\hline Solvent & & Moment (Debye) \\
\hline Water & 1.85 \\
\hline Methanol & 1.70 \\
\hline Ethanol & 1.69 \\
\hline Isopropanol & & 1.66 \\
\hline
\end{tabular}

First, $5 \mathrm{~mL}$ of the alcohol being tested were added to each sample to pretreat the pelletized algae. This pretreatment step was meant to test the ability of the alcohols to free lipids from algae cells in the samples. The addition and mixing of the alcohols was a low-energy process which may accomplish the same task as sonication without sacrificing scalability. The tubes containing the algae were then allowed to warm to room temperature, allowing the alcohol time to penetrate the algae cells in the samples. After 15 minutes of pretreatment, the samples were transferred from the centrifuge tubes to glass test tubes. 
Deionized water $(4 \mathrm{~mL})$ was added to each sample. The water was added first to the original centrifuge tube that had contained each sample and then transferred into the glass tube which contained the sample at this point. This step was taken in order to recover any residual algae from the centrifuge tubes.

Hexane $(2 \mathrm{~mL})$ was added to each tube and the tubes were hand mixed for 15 seconds. The mixtures were then given approximately five minutes to separate into layers before the hexane layer, which contained the extraction lipids, was transferred by pipette to a tared glass test tube. An additional $2 \mathrm{~mL}$ of hexane were added, mixed and transferred. The purpose of performing the hexane addition and transfer two times was to achieve nearly the maximum possible removal of lipids from the sample.

The extracts were dried, as in the lipid production experiment, according to the vessels in which they were contained. Again, mass and identity were measured. Six algae pellets were extracted with each of the four solvent systems (methanol-hexane, ethanol-hexane, isopropanol-hexane and Bligh and Dyer). All 24 of the pellets used in this experiment were derived from the sample algae sample, collected on Day 9. For each solvent system, three pellets analyzed gravimetrically to obtain lipid mass and three pellets were analyzed chromatographically for triglyceride identification.

Some sample vials during alcohol extraction are shown in Figure 14 directly after the first addition of hexane. A methanol-hexane sample is on the left, an ethanol-hexane sample is in the middle and an isopropanol-hexane sample is on the right. 


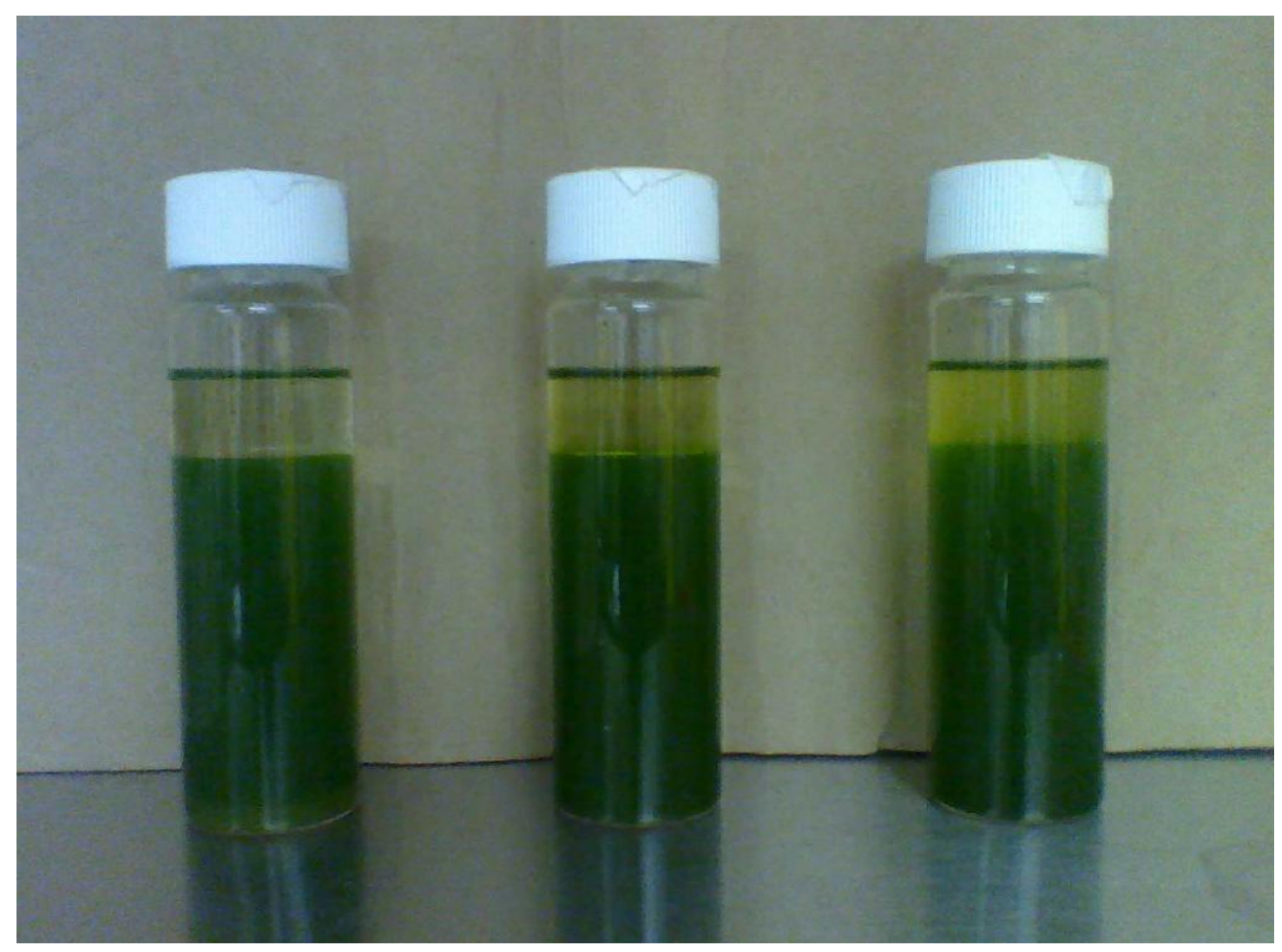

Figure 14: Alcohol-hexane extractions (methanol, ethanol, isopropanol, left to right)

\section{Quality Control}

Several quality control tests were undertaken to verify the accuracy and repeatability of the extraction procedures investigated in the present research. Quality control measures included the performance of blank extractions, control extractions using known quantities of oil, and matrix spikes.

The blank extractions were conducted by performing all of the steps of a normal extraction on a clean test tube without a sample in it. This procedure is used to reveal 
any error caused by the extraction procedure. The absence of a sample in the tube means that no mass should be produced by the extraction.

Blank extractions were conducted using each of the extraction procedures described above, including the Bligh and Dyer procedure. The Bligh and Dyer method was the only procedure that had a detectable error. The blank Bligh and Dyer extraction produced $0.2 \mathrm{mg}$ of material. In an actual Bligh and Dyer extraction of a sample, the mass of this material would have been erroneously included in the lipid fraction. The erroneous $0.2 \mathrm{mg}$ would have been equivalent to $8.3 \%$ of the mass of the single lowestyielding sample from the entire experiment, which is a minor error. This suggests that the lipids measured by the Bligh and Dyer procedure in the experiments were can be considered to have been derived from the algae.

Control extractions are performed by adding a known mass of oil to a test tube and performing an extraction on it. Ideally, the mass of oil added is equal to the mass produced by the extraction. Vegetable oil was used as the control material. This procedure was performed on methanol-hexane, ethanol-hexane, isopropanol-hexane, and Bligh and Dyer extraction procedures. The extractions had negative errors of $1.32 \%$, $1.37 \%, 1.02 \%$, and $2.37 \%$, respectively. The errors could have been due to inefficient partitioning of the oil into the nonpolar layers during the extractions.

A matrix spike is a quality control measure in which a known mass of oil is added to a sample and an extraction performed on the mixture. This test confirms that the sample matrix does not interfere with experimental accuracy. Once again, vegetable oil was used to spike the sample. The matrix spike test was conducted only on the Bligh and Dyer 
method. Matrix spikes were omitted for the alcohol-based extraction methods.

In order to perform a matrix spike on the Bligh and Dyer procedure, three samples were prepared in centrifuge tubes, as in the normal extraction experiments. Two of the tubes were used for normal Bligh and Dyer extractions. This made possible an estimation of the native concentration of lipids in the sample to be spiked. A mass of vegetable oil approximately three times the expected native mass was added to the spike sample. This effectively quadrupled the mass of oil in the test tube. The spike resulted in a negative error of $6.14 \%$. 


\section{Chapter 4: Results and Discussion}

This chapter describes the results of the lipid production experiment and the extraction procedure experiment.

\section{Lipid Production Experiment}

The batch-mode algae pond was operated for a 3.5 week period from March 23 through April 17, 2009 on municipal wastewater effluent. In addition to the results of laboratory analysis, the daily upkeep of the pond led to several insights about the setup and operation of a batch-mode algae pond.

\section{General Observations}

Between the first and second day of algae pond operation, the concentration of algal mass in the pond decreased, before rebounding on the third day. This indicates that an initial period of settling took place in spite of the mixing action of the paddle wheel.

The pond was initially dark yellow in color and became visibly greener after four days of operation. This corresponded with the beginning of the rapid growth of the algae as determined by solids analysis.

\section{Algae Identification}

The composition of the algae population changed over the course of the batch-mode experiment. Microscopic investigations were performed on the inoculum and growth medium as well as on the pond water throughout the experiment. 
The water used to inoculate the pond had been grown in a continuous-mode pond fed by the same source as the batch-mode pond. Dictyosphaerium was the predominant genus of algae in the water used to inoculate the pond, followed by Nitzschia. Dictyosphaerium a contributed an estimated $90 \%$ of the biomass of the inoculum, while Nitzschia accounted for approximately $10 \%$.

The wastewater that was used as the growth medium was also examined by microscope. No algae were apparent in the growth medium. The biomass consisted mostly of rodshaped bacteria (Figure 15).

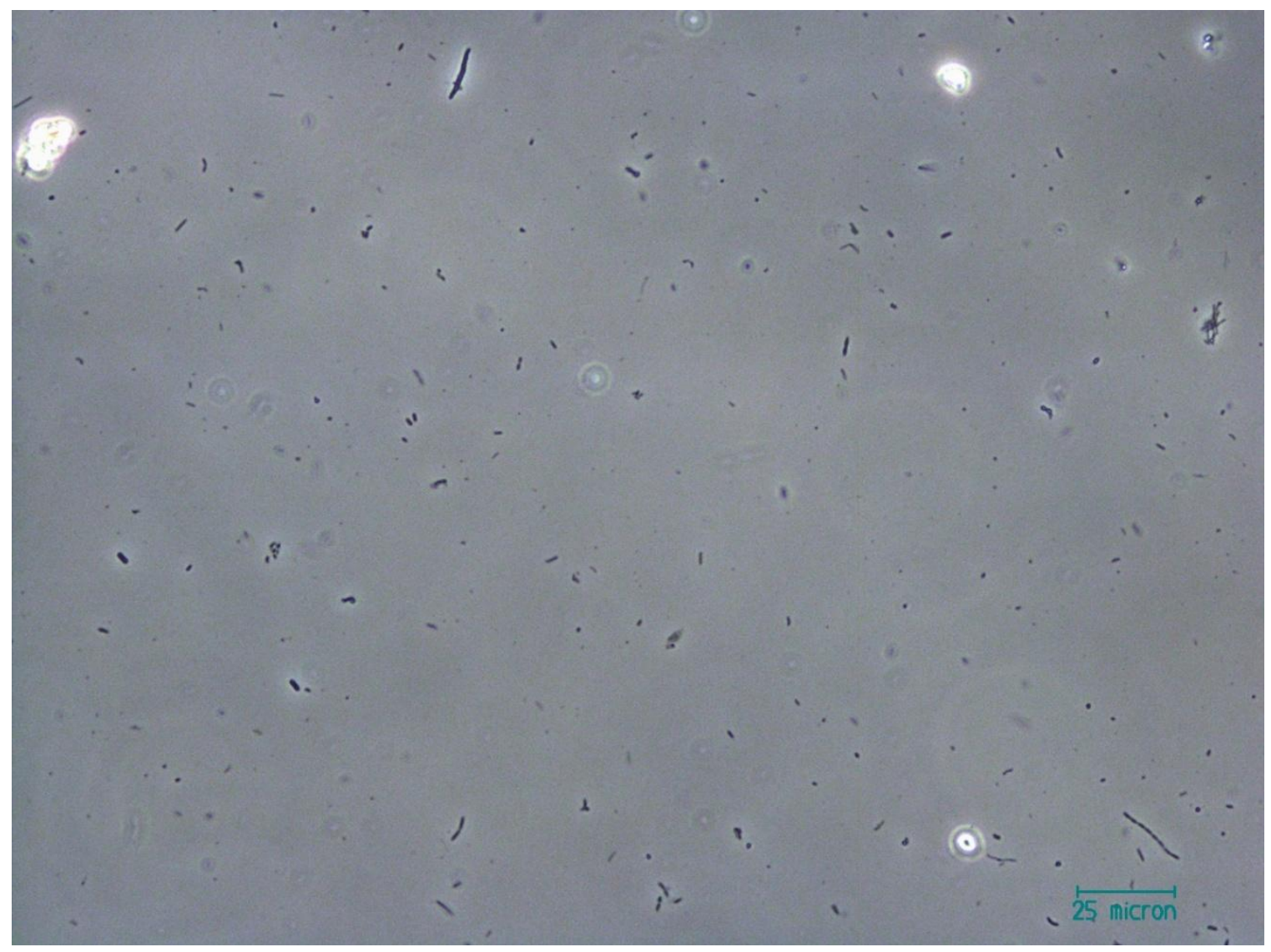

Figure 15: 400X Micrograph of primary wastewater effluent used in algae pond 
After five days, the dominant alga was still Dictyosphaerium. Nitzschia numbers had increased by this point to roughly $15-20 \%$ of the biomass in the water sampled. Golenkinia were also observed in very small numbers.

After ten days, the diversity of the culture had expanded to include several new genera. Dictyosphaerium remained the most populous, at approximately $50 \%$ of the population. Nitzschia accounted for approximately $20 \%$ of the culture. Scenedesmus made up about $15 \%$ of the population, while Micractinium, Actinastrum and Ankistrodesmus each made up about 5\% of the population.

After fifteen days, the Dictyosphaerium, Nitzschia and Scenedesmus had become approximately equally populous. Micractinium, Actinastrum and Ankistrodesmus were still present, but were not as well represented as the aforementioned three genera.

One week later, after twenty-two days, the culture had begun to decline, as was readily apparent by microscopic investigation. Many of the algal cells were broken and a great deal of debris was visible throughout the sample. The predominant microalgae were Dictyosphaerium, Nitzschia and Scenedesmus; all were present in approximately equal numbers.

Some example images of the culture throughout the experiment are shown below. The first image shows the inoculum, grown in continuous mode at a five day residence time. The spherical algae Dictyosphaerium was present in the inoculum and remained the dominant species throughout the experiment. The next image was taken from the pond on Day 9 and shows Dictyosphaerium and Scenedesmus. Scenedesmus appeared shortly after the experiment was initiated and remained for the duration of the life of the culture. 
The next image was taken on Day 14 and illustrates the increasing diversity of the culture that came with time. The image includes Actinastrum as well as Scenedesmus and Dictyosphaerium. The last image was captured on Day 21 and shows the cellular debris that started to accumulate in the pond as the algae culture declined.

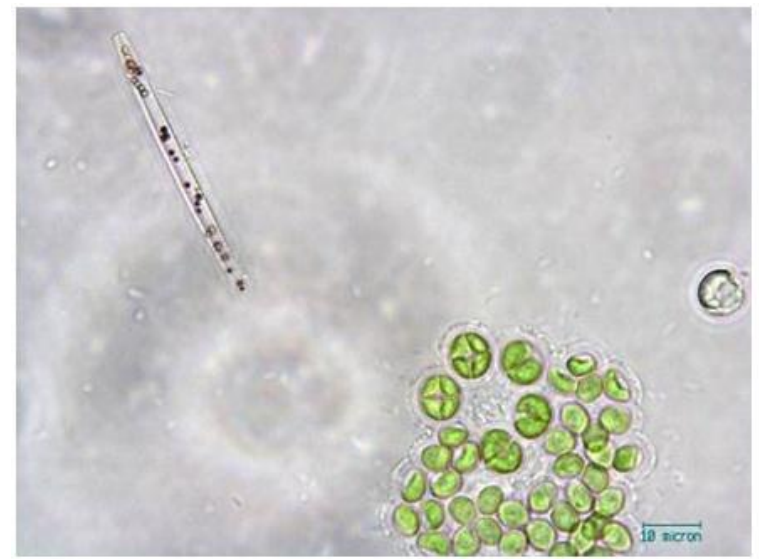

Inoculum

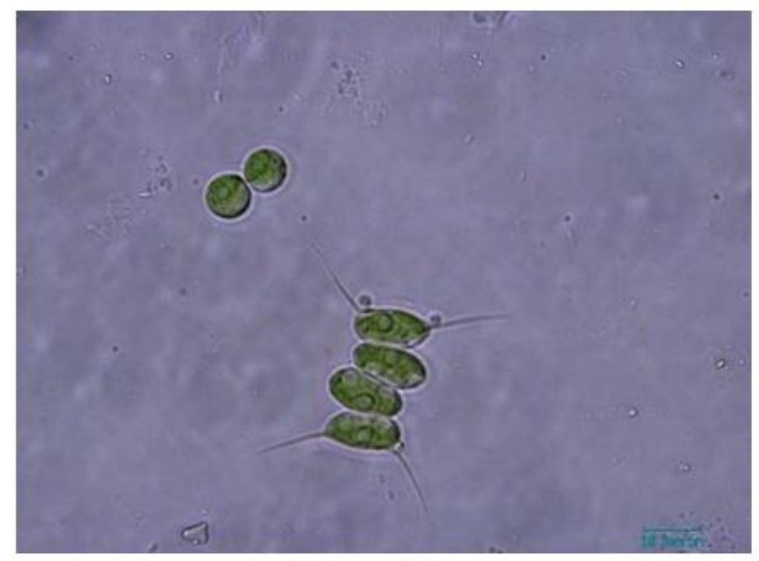

Day 14

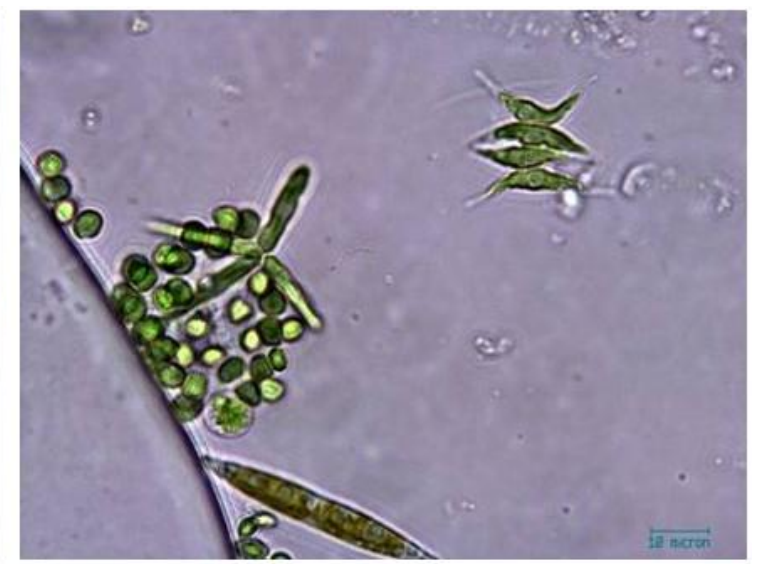

Day 9

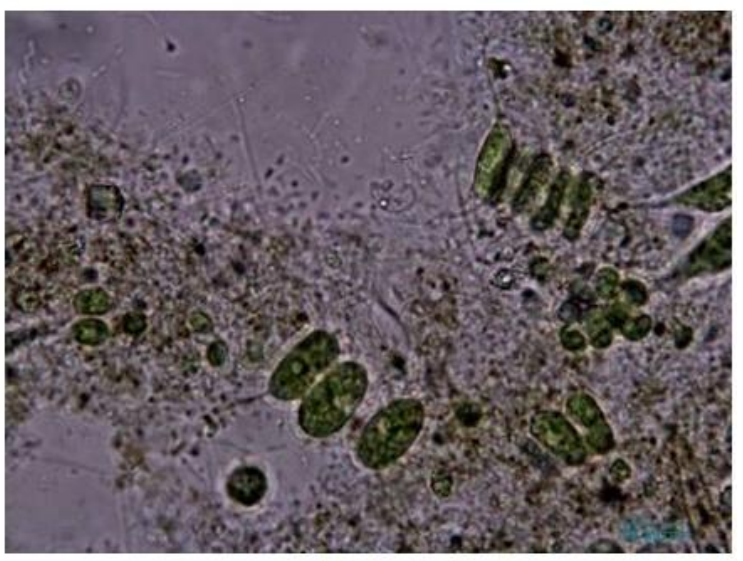

Day 21

Figure 16: 1000X algae culture micrographs 


\section{Algae Growth}

The total and volatile suspended solids of the inoculum were $212 \mathrm{mg} / \mathrm{L}$ and $175 \mathrm{mg} / \mathrm{L}$, respectively. The total and volatile suspended solids of the wastewater, which was used as a growth medium for the pond, were $80 \mathrm{mg} / \mathrm{L}$ and $72 \mathrm{mg} / \mathrm{L}$, respectively.

After inoculation, the growth of the algae culture did not exactly resemble a typical fourphase batch growth curve. Total and volatile suspended solids were measured on each day of the 25 day lipid production experiment. After a brief decline between Day 1 and Day 2, the volatile suspended solids began growing rapidly, peaking at $527 \mathrm{mg} / \mathrm{L}$ on Day 17. Over much of the growth phase, concentrations increased linearly instead of in the classical exponential shape of batch growth. This decelerated growth was probably caused by light limitation due to cell self-shading. After reaching the maximum concentration, volatile solids began an unsteady decline from Day 17 to Day 25, at which point the sampling ceased. The classical stationary phase of the batch growth curve was not clearly present. The graph in Figure 17 illustrates the development of total and volatile solids over the life of the pond culture.

The error bars in the following graphs represent the standard deviation of triplicate analyses of splits of single samples collected from the pond. 


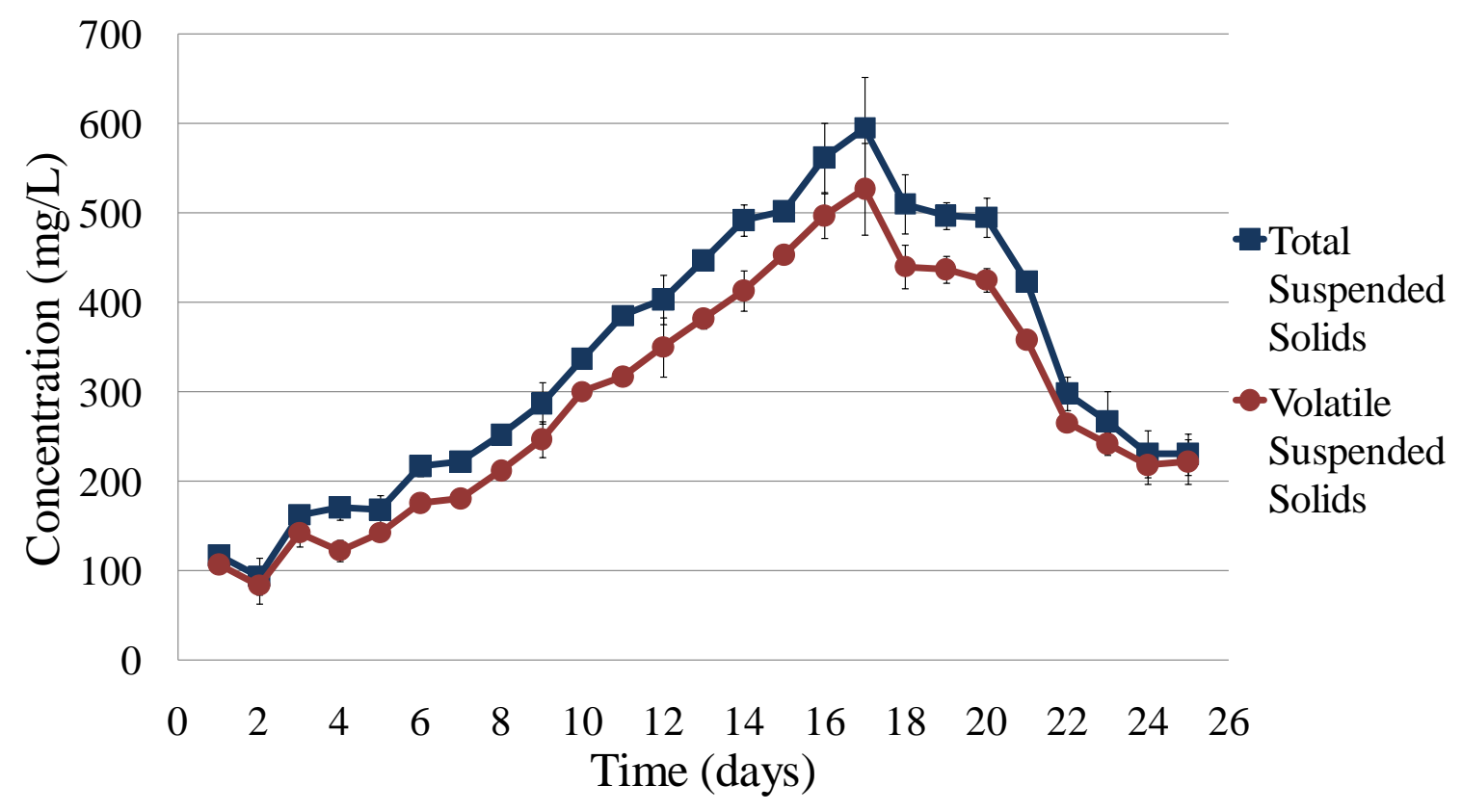

Figure 17: Total and volatile suspended solids development of batch culture

An average growth rate of approximately $32 \mathrm{mg} / \mathrm{L} /$ day of volatile suspended solids was observed during the period of rapid growth between the seventh and seventeenth days of operation of the pond. The maximum daily growth rate of volatile suspended solids was $53.3 \mathrm{mg} / \mathrm{L} /$ day $\left(10.7 \mathrm{~g} / \mathrm{m}^{2} / \mathrm{d}\right)$ occurring during Days 9 and 10 of the experiment when total volatile solids concentrations ranged from $250-300 \mathrm{mg} / \mathrm{L}$. From the period of time between the inoculation and the initial decline of the pond, the average rate of volatile suspended solids increase was approximately $26 \mathrm{mg} / \mathrm{L} /$ day.

The $\mathrm{pH}$ of the pond was held fairly constant by manually-controlled $\mathrm{CO}_{2}$ addition, but on Days 10,12 , and 16 , the $\mathrm{pH}$ reach nearly 9 , which is high enough to potentially slow algal growth Figure 18. 


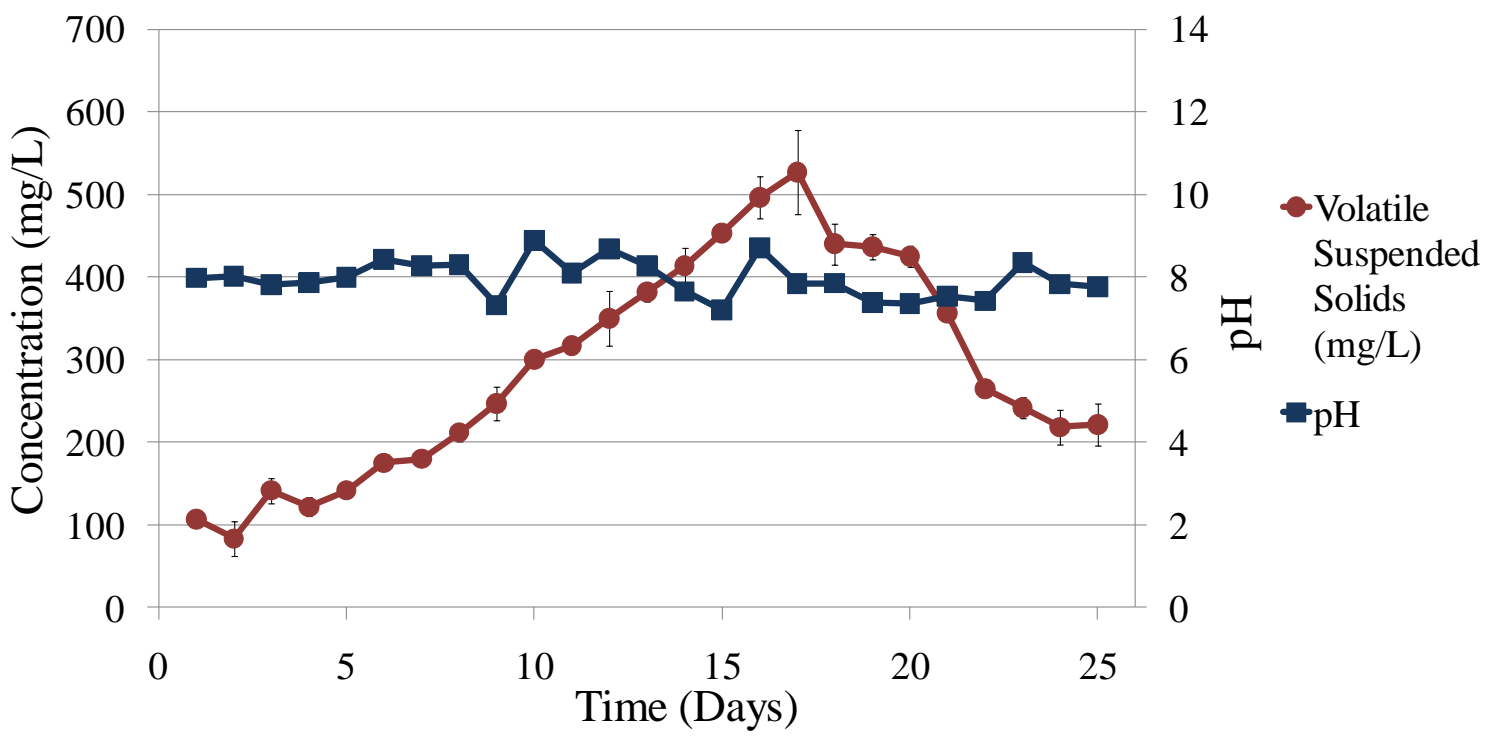

Figure 18: Daily volatile suspended solids and $\mathrm{pH}$

\section{Lipid Development}

The inoculum volatile suspended solids contained $17.1 \%$ lipids, and the volatile suspended solids of the wastewater contained $14.9 \%$ lipids by weight, both determined by the gravimetric method. Subsequent to the inoculation of the algae pond, lipid measurements were taken regularly. Lipid fractions were determined gravimetrically and by gas chromatography. The extracts analyzed by gravimetry and gas chromatography were both prepared using the Bligh and Dyer extraction method.

The lipid concentrations obtained by the gravimetric method included any nonpolar material extracted into the chloroform during the Bligh and Dyer procedure, such as lipids and chlorophyll. The concentrations obtained by the chromatographic method 
included only fatty acid methyl esters extracted into hexane after the Bligh and Dyer procedure. Thus, the values obtained by the gravimetric method and chromatographic method differ.

Although the lipid concentration values obtained by the gravimetric method include a broader variety of compounds than the chromatographic method, the values obtained by gravimetry were consistently lower. The maximum daily growth rate obtained by the gravimetric method was approximately $8.1 \mathrm{mg} / \mathrm{L} /$ day, or about $1.65 \mathrm{~g} / \mathrm{m}^{2} /$ day. The average daily lipid production over the period of rapid culture growth between Day 7 and Day 17 was approximately $2.5 \mathrm{mg} / \mathrm{L} /$ day, or about $0.52 \mathrm{~g} / \mathrm{m}^{2} /$ day.

The lipid production measured by the chromatographic method was higher. The concentration of fatty acids in the growth medium by gas chromatography ranged from $13 \mathrm{mg} / \mathrm{L}$ on the Day 2 to $104 \mathrm{mg} / \mathrm{L}$ on the Day 11 . Because some of the daily concentrations of specific fatty acids were obscured by contaminants in the samples, it is difficult to determine the maximum daily growth rate. However, based only on fatty acids, which were definitively measure in both Day 11 and Day 13 samples, an experiment high daily growth rate of about $21.7 \mathrm{mg} / \mathrm{L} /$ day, or about $4.40 \mathrm{~g} / \mathrm{m}^{2} / \mathrm{day}$, was measured. It is not expected that a radical change in this value would be observed upon inclusion of the missing acids. The average daily fatty acid production over the period of culture growth between Day 4 and Day 17 was approximately $4.8 \mathrm{mg} / \mathrm{L} / \mathrm{day}$, or about $0.97 \mathrm{~g} / \mathrm{m}^{2} /$ day. 


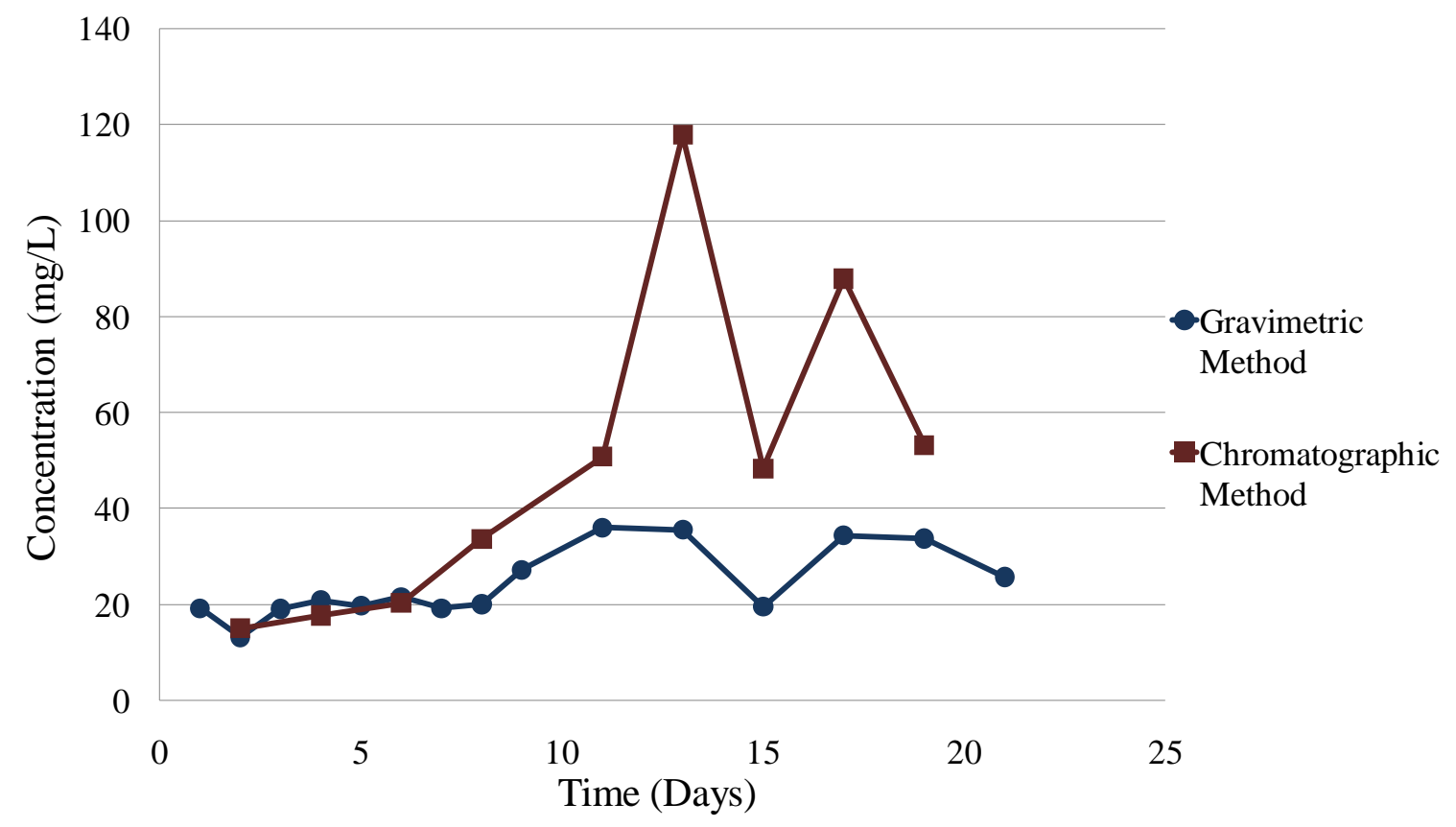

Figure 19: Comparison of gravimetric and chromatographic methods of lipophilic compound analysis throughout batch growth

The following graphs show the development of lipids over time by the gravimetric method and the development of fatty acids over time by the chromatographic method. 


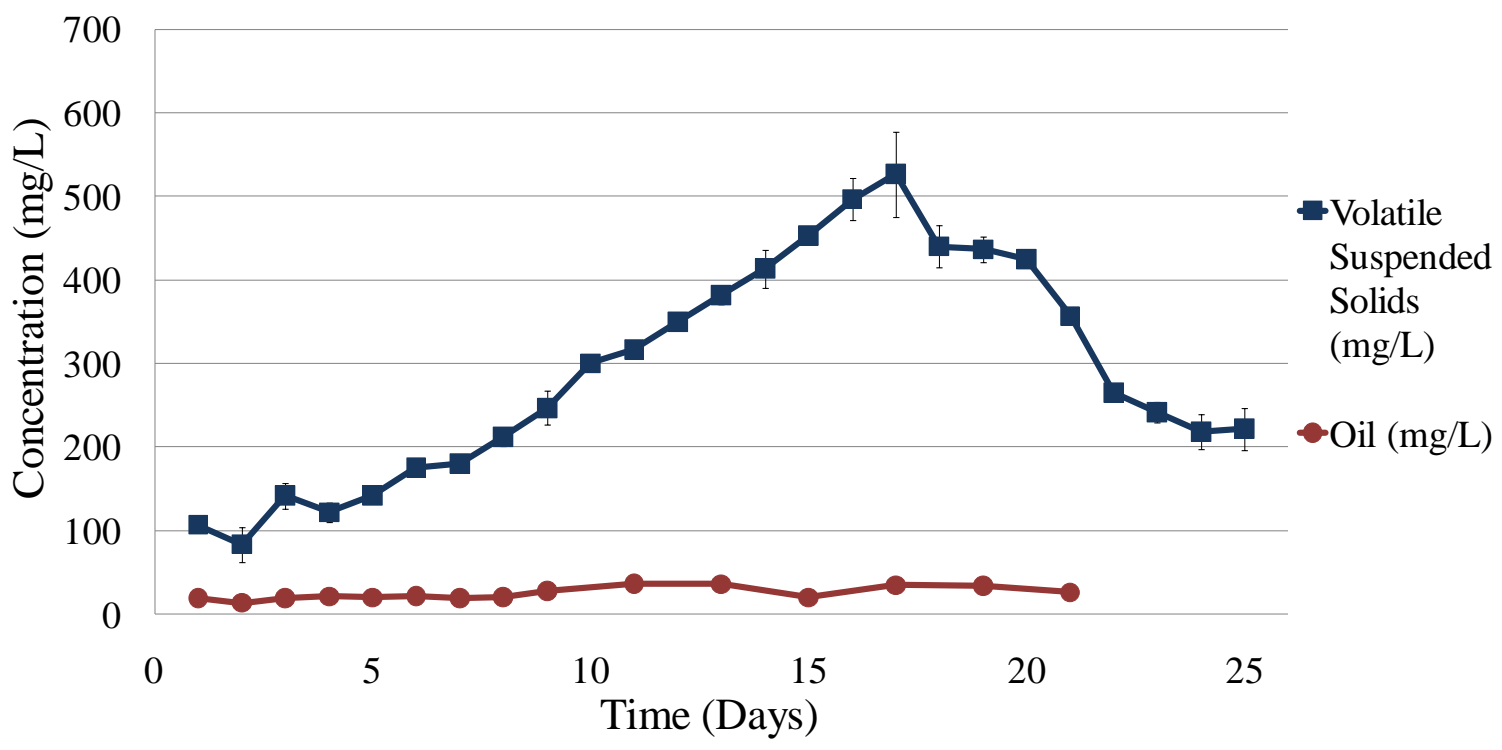

Figure 20: Lipid development in batch culture algae as determined by the gravimetric method

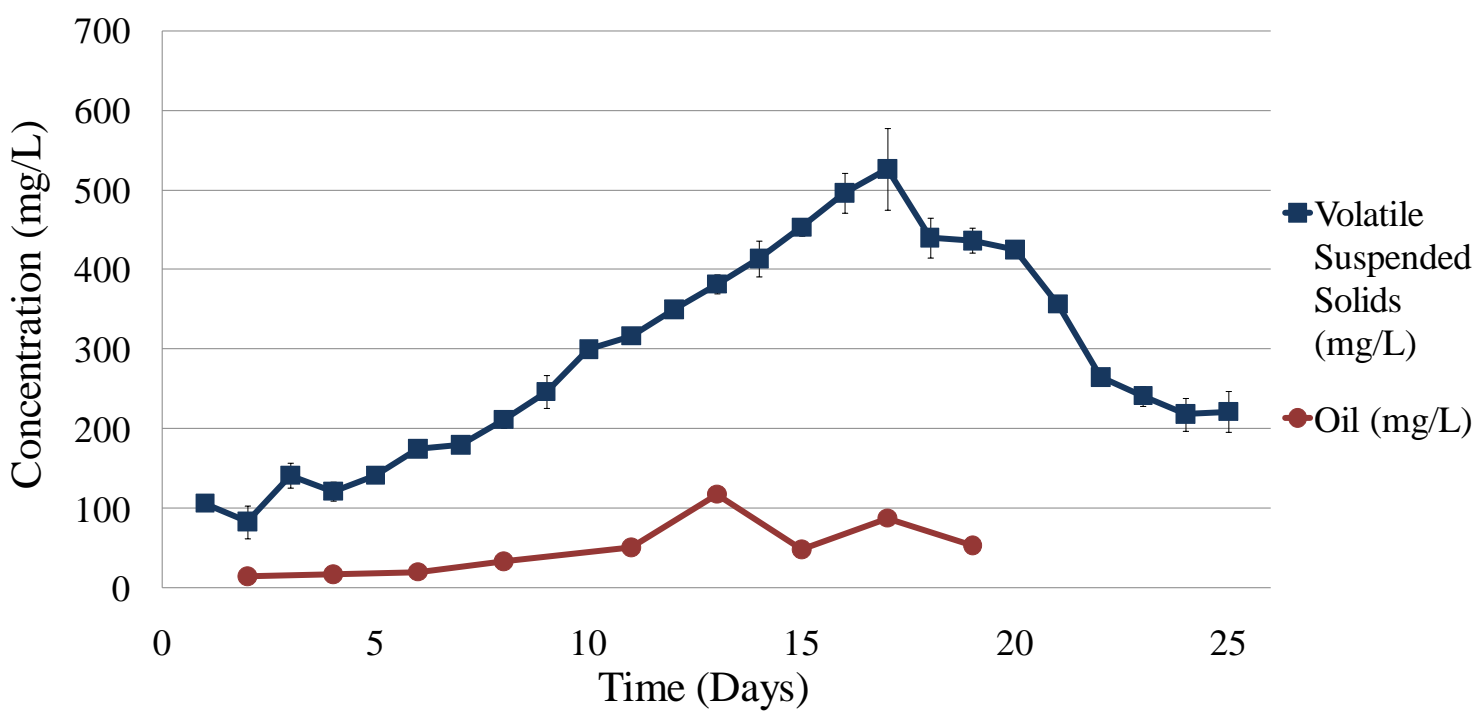

Figure 21: Fatty acid development in batch culture algae as determined by the chromatographic method 
Although the concentrations of fatty acid and lipid material were not very similar, their proportional correspondence was very apparent. Similar patterns of oil growth and subsidence were observed using both methods. Both of the graphs also reflect a sharp decline in lipid concentration that occurred on ninth and fifteenth days of operation of the pond. This decline may have been due to a problem with the sampling technique as it is apparent in both the gravimetric and chromatographic tests, which were each conducted on the same sample of pond water.

The maximum growth rate of lipids occurred within the growth period. This general observation is supported by both the gravimetric and chromatographic data and also agrees with the findings of Woertz (2007), who performed a similar experiment using exclusively the gravimetric method.

\section{Fatty Acid Analysis}

This section discusses the results of the chromatographic analyses performed throughout this research.

Inoculum and Growth Medium

Chromatographic analyses of the inoculum and the growth medium were conducted prior to their addition to the algae pond. Data pertaining to the fatty acid content of each are presented in Table 3. Data on 9-octadecanoic acid methyl ester (Z) are not reported for the inoculum because they could not be assessed accurately due to contamination in the sample. 
Table 3: Fatty acid concentrations $(\mathrm{mg} / \mathrm{L})$ in inoculum and initial wastewater growth medium (dashes represent fatty acids that were not measured).

\begin{tabular}{lccccc} 
& $\mathrm{C} 16: 1$ & $\mathrm{C} 16$ & $\mathrm{C} 18: 1$ & $\mathrm{C} 18$ & Total \\
\cline { 2 - 6 } Inoculum & 10.6 & 10.7 & - & 8.2 & 29.5 \\
Growth Medium & 0.5 & 2.9 & 5.6 & 2.9 & 11.8
\end{tabular}

Fatty Acids throughout Batch Growth

In addition to determining the overall mass of fatty acids by gas chromatography, measurements were made of the contribution of each type of individual fatty acid to the total (Figure 21). Four fatty acid methyl esters were observed in most of the samples produced throughout the experiment. The completely saturated hexadecanoic acid methyl ester (C16) was the most common (Table 4). The cis-bonded, monounsaturated 9-hexadecanoic acid methyl ester $(\mathrm{Z})(\mathrm{C} 16: 1)$ was the second most common. The third most common acid was 9-octadecanoic acid methyl ester $(\mathrm{Z})(\mathrm{C} 18: 1)$, followed by the saturated octadecanoic acid methyl ester (C18). Although none of the compounds was ever present in concentration significantly larger than the other three, this hierarchy of concentrations was consistent throughout the life of the algal culture.

Figure 22 compares the volatile suspended solids concentration of the pond to the fraction of volatile suspended solids which are composed of fatty acids. The data on which the graph is based were taken from the chromatographic experiments. 


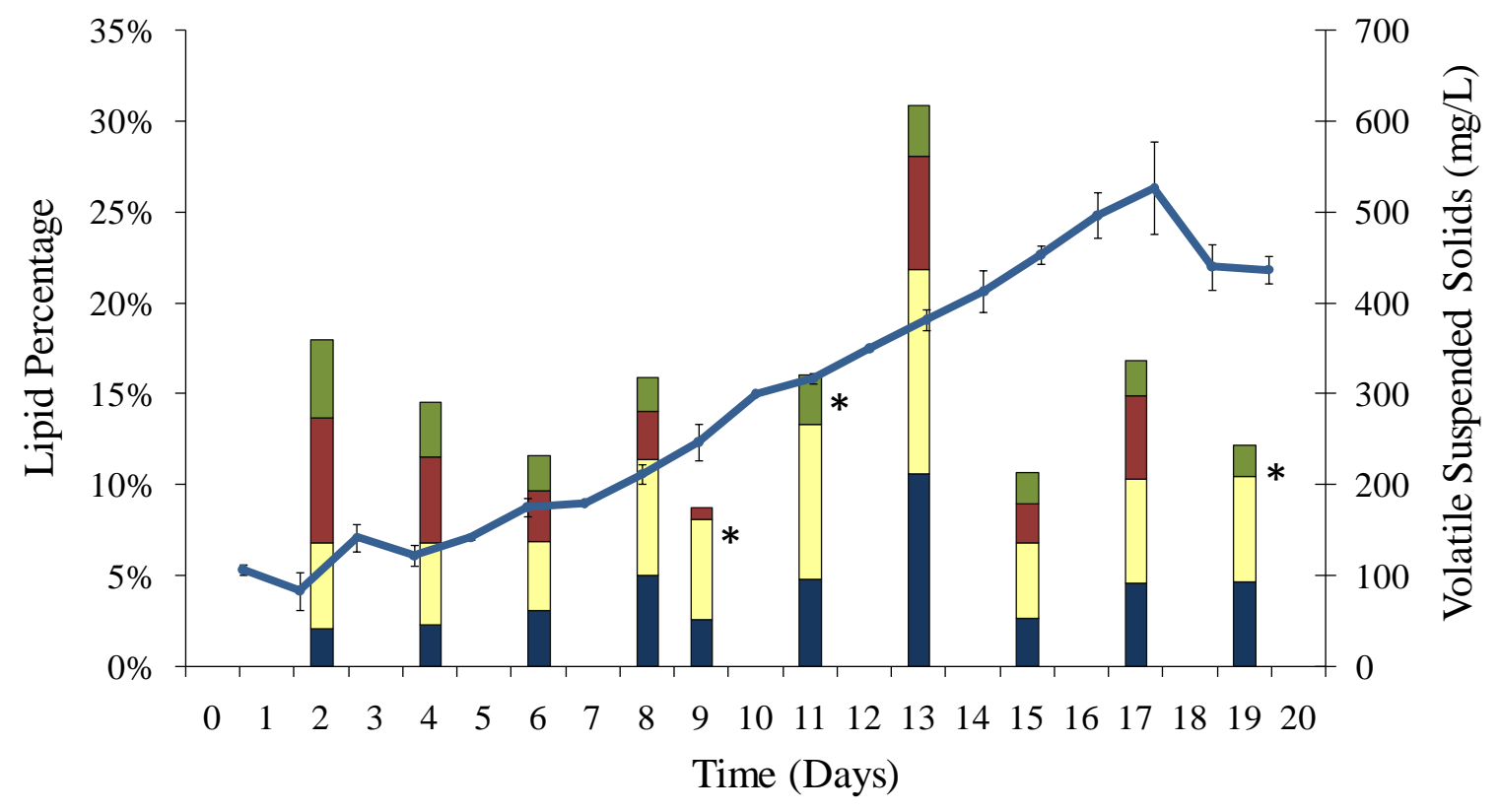

$\square \mathrm{C} 16: 1 \square \mathrm{C} 16 \quad \square \mathrm{C} 18: 1 \quad \square \mathrm{C} 18 \quad$ - Volatile Suspended Solids

* Certain fatty acids are excluded from several data points due to uncertainties caused by coeluting contaminants.. This does not necessarily mean that these fatty acids were absent from the samples.

Figure 22: C16 and C18 fatty acids as percentage of volatile suspended solids throughout batch growth

A typical chromatogram of the wastewater algae extracts is pictured below with the prominent fatty acid constituents labeled. The chromatogram was taken from a sample of the batch-mode pond collected on Day 11. It includes all four of the common fatty acids discussed previously and the odd-chain fatty acids used as internal standards: pentadecanoic acid methyl ester (C15) and nonadecanoic acid methyl ester (C19). 


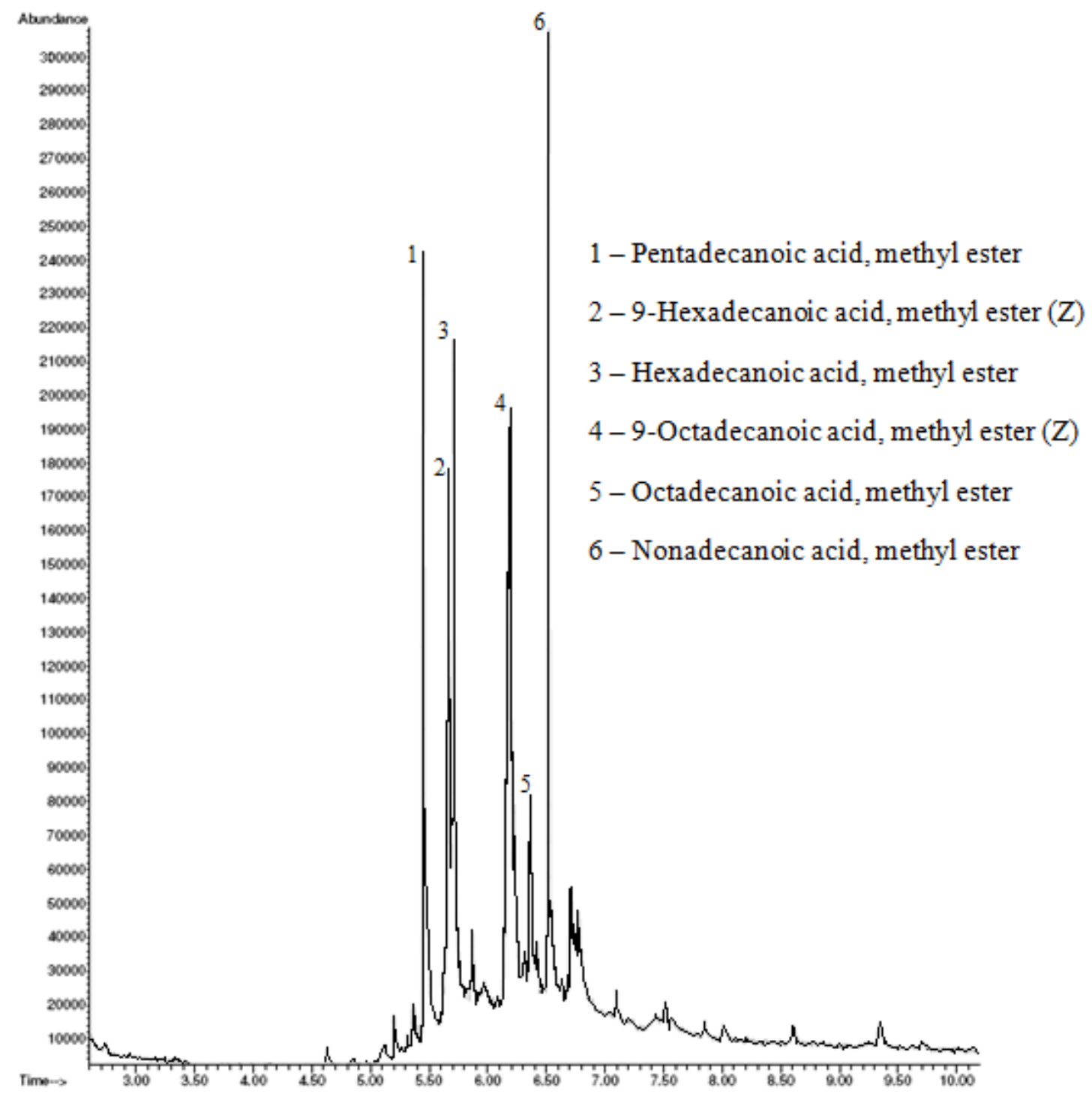

* Peak 1 and Peak 6 are internal standards. All other labeled peaks are native to the sample.

Figure 23: Typical chromatogram of a transmethylated algal extract with internal standards pentadecanoic acid methyl ester and nonadecanoic acid methyl ester

The graph in Figure 24 depicts the concentrations of the four common constituent fatty acids over the duration of the operation of the batch-mode pond. Points at which the lines of the graph are broken do not reflect a concentration of zero. Data points were 
omitted in these cases because contamination in the samples prevented the accurate assessment of the concentration of coeluting fatty acid methyl esters.

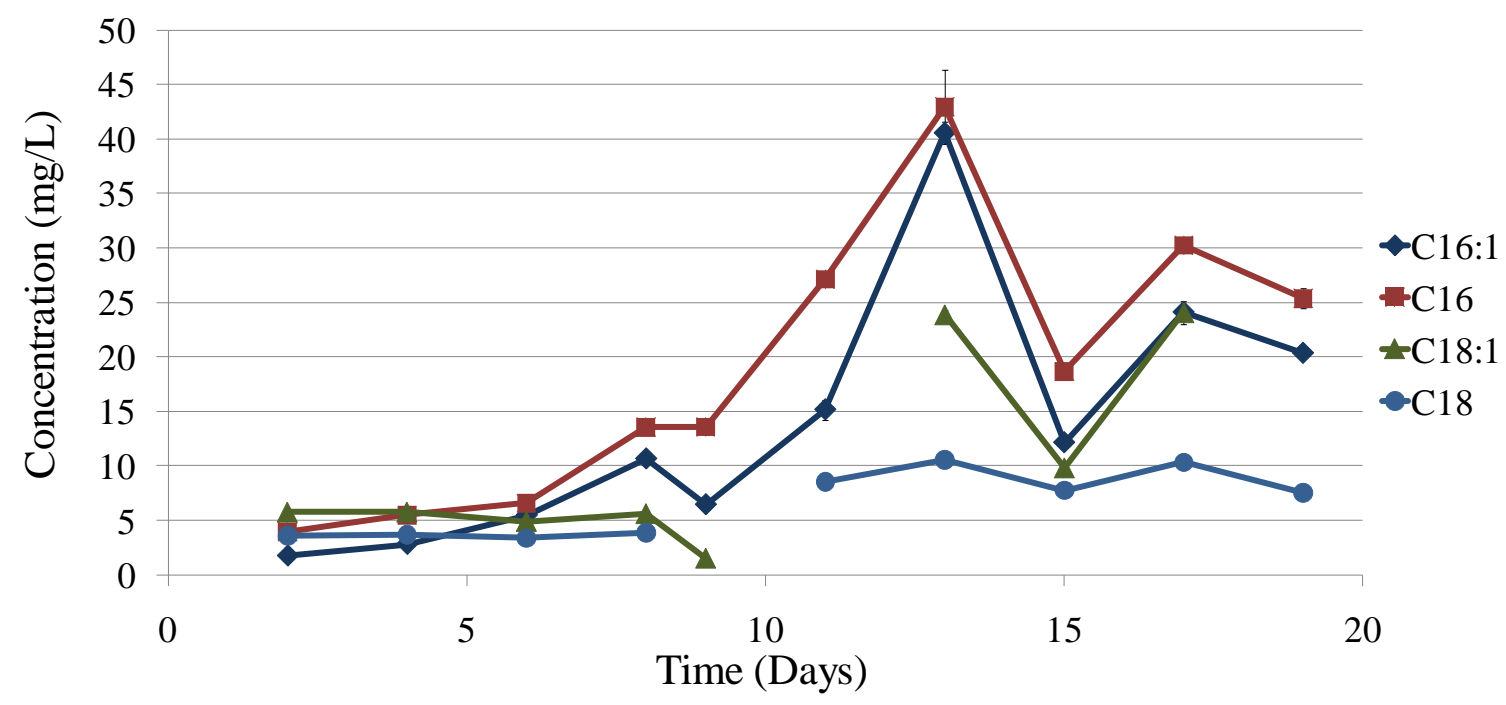

Figure 24: Concentration of fatty acids in wastewater medium during batch growth

The contribution of each individual fatty acid to the total mass of fatty acids changed over the course of the operation of the algae pond (Table 4). The fraction of fatty acid mass contributed by 9-hexadecanoic acid methyl ester (Z) increased steadily over the course of the experiment, beginning at $12 \%$ and ending at $27 \%$ on Day 17 . Hexadecanoic acid methyl ester started at $26 \%$ and reached a peak of $36 \%$ on Day 13 before declining to $34 \%$ on Day 17 . The contribution of 9-Octadecanoic acid methyl ester $(Z)$ decreased steadily over the course of the experiment from $38 \%$ on Day 2 to $27 \%$ on Day 17. The portion of octadecanoic acid methyl ester also declined steadily from $24 \%$ on Day 2 to $11 \%$ on Day 17 . 
On days that did not produce reliable data, some values are omitted. As was described in detail in the Materials and Methods section, in some cases, the coelution of contaminants with fatty acid methyl esters prevented reliable conclusions from being made on certain fatty acid methyl ester concentrations. In Table 4, unreliable data are omitted (hyphens). The other fatty acid methyl esters that occurred on the same day as the unreliable data points are shown in the table as percentages of the total fatty acid methyl esters reliably analyzed on those days.

Table 4: Individual fatty acids as fraction of whole as determined by Bligh and Dyer

\begin{tabular}{lcccc} 
& $\mathrm{C} 16: 1$ & $\mathrm{C} 16$ & $\mathrm{C} 18: 1$ & $\mathrm{C} 18$ \\
\cline { 2 - 5 } Day 2 & $12 \%$ & $26 \%$ & $38 \%$ & $24 \%$ \\
Day 4 & $16 \%$ & $31 \%$ & $33 \%$ & $21 \%$ \\
Day 6 & $27 \%$ & $32 \%$ & $24 \%$ & $17 \%$ \\
Day 8 & $32 \%$ & $40 \%$ & $17 \%$ & $11 \%$ \\
Day 9 & $30 \%$ & $63 \%$ & - & - \\
Day 11 & $30 \%$ & $53 \%$ & - & $17 \%$ \\
Day 13 & $34 \%$ & $36 \%$ & $20 \%$ & $9 \%$ \\
Day 15 & $25 \%$ & $39 \%$ & $20 \%$ & $16 \%$ \\
Day 17 & $27 \%$ & $34 \%$ & $27 \%$ & $11 \%$ \\
Day 19 & $38 \%$ & $48 \%$ & - & $14 \%$
\end{tabular}

\section{Common Contaminants}

A number of contaminants were observed frequently in the samples that were prepared for chromatographic analysis. Dibutyl phthalate appeared several times. Dibutyl phthalate is a common plasticizer used in the production of inks, adhesives, soft plastics 
and insect repellents (New Jersey Department of Health and Senior Services, 2001). It is likely that it was present because it was leached from a plastic centrifuge tube or cap during the workup of the chromatographic samples. Because it is not very reactive, it is not suspected of interfering significantly with fatty acid methyl ester analysis (New Jersey Department of Health and Senior Services, 2001).

Dibutyl sebacate was also a common contaminant. Dibutyl sebacate is a food grade plasticizer used in the production of soft plastics, synthetic rubbers and as a flavor additive (International Programme on Chemical Safety, 2001). It is likely that the dibutyl sebacate present in the samples was leached from a plastic centrifuge tube or cap during the workup of the chromatographic samples. Due to a very low reactivity, it is not expected that dibutyl sebacate interferes significantly with fatty acid methyl ester analysis (International Programme on Chemical Safety, 2001).

\section{Extraction Procedure Experiment}

The extraction procedure experiment was performed on samples collected on Day 9 from the batch-mode pond to investigate extraction methods. Three alcohol-based solvent systems, methanol, ethanol and isopropanol, were tested in a simple, industriallypracticable extraction procedure. The solvents were compared to a Bligh and Dyer extraction which was performed on an aliquot of the same sample.

\section{Extraction Effectiveness by Solvent System}

Of the four extraction procedures tested, the Bligh and Dyer method extracted the greatest mass of material from the sample, followed by the methanol-hexane system, 
ethanol-hexane, and isopropanol-hexane at $84 \%, 54 \%$ and $49 \%$ of the Bligh and Dyerextracted mass, respectively (Figure 25). The methanol-hexane solvent extraction removed nearly as much material from the sample as did the relatively involved Bligh and Dyer extraction.

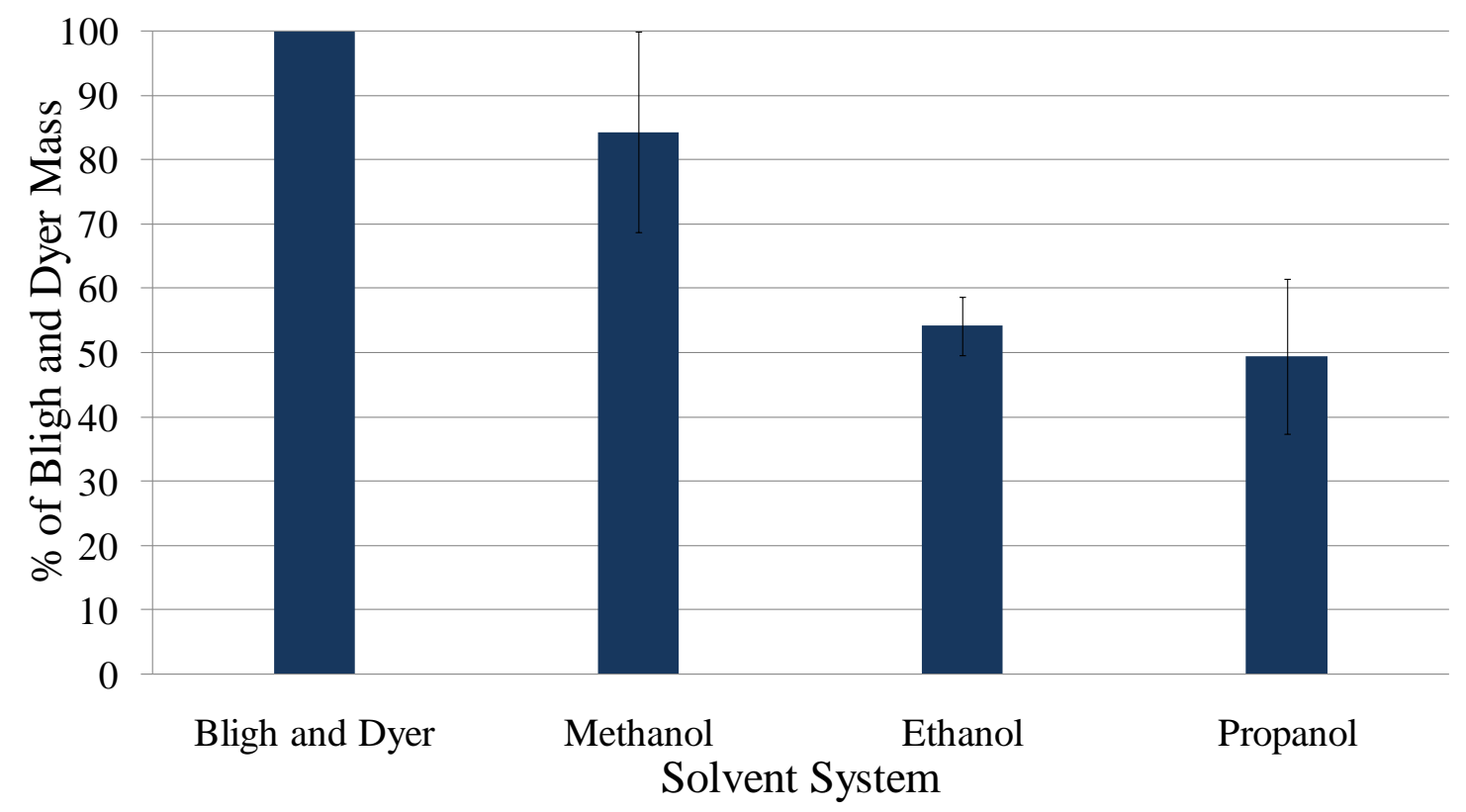

Figure 25: Extraction effectiveness of each solvent system as percentage of Bligh and Dyer-extracted mass

\section{Individual Fatty Acid Methyl Esters Extracted by Each Solvent}

\section{System}

Although methanol extracted the most mass of any of the alcohol-based solvent systems, it extracted the smallest mass of triglycerides (Figure 26). The isopropanol-hexane 
system, despite extracting the smallest mass of any solvent system tested, extracted the most fatty acids of any of the alcohol-based solvent systems. The isopropanol-hexane system extracted nearly as much hexadecanoic acid and 9-hexadecanoic acid (Z) as the Bligh and Dyer extraction system; $82 \%$ and $84 \%$, respectively. The ethanol-hexane system followed, extracting $36 \%$ and $34 \%$ respectively of the hexadecanoic acid and 9hexadecanoic acid $(Z)$ that the Bligh and Dyer procedure produced. The methanolhexane system removed the smallest mass of fatty acids of all of the systems tested at $23 \%$ of the hexadecanoic acid and $23 \%$ of the 9-hexadecanoic acid (Z) of the Bligh and Dyer system.

Previous research has shown that cellular walls and hydrophilic components of outer cellular membranes can impede contact between lipophilic solvents and intracellular compounds (Sikkema, Bont, \& Poolman, 1995), (Hejazi, 2004). The effectiveness of isopropanol as counter-solvent is probably due to its relative affinity for lipophilic cell wall and cell membrane components compared with more highly polar solvents such as ethanol and methanol. Although isopropanol is polar, it is more likely than ethanol or methanol to be partitioned into the lipid bilayer of a cell, from where it may promote contact between nonpolar solvents and cellular products (Sikkema, Bont, \& Poolman, 1995). Figure 26 shows the mass of fatty acids extracted by each solvent system. 


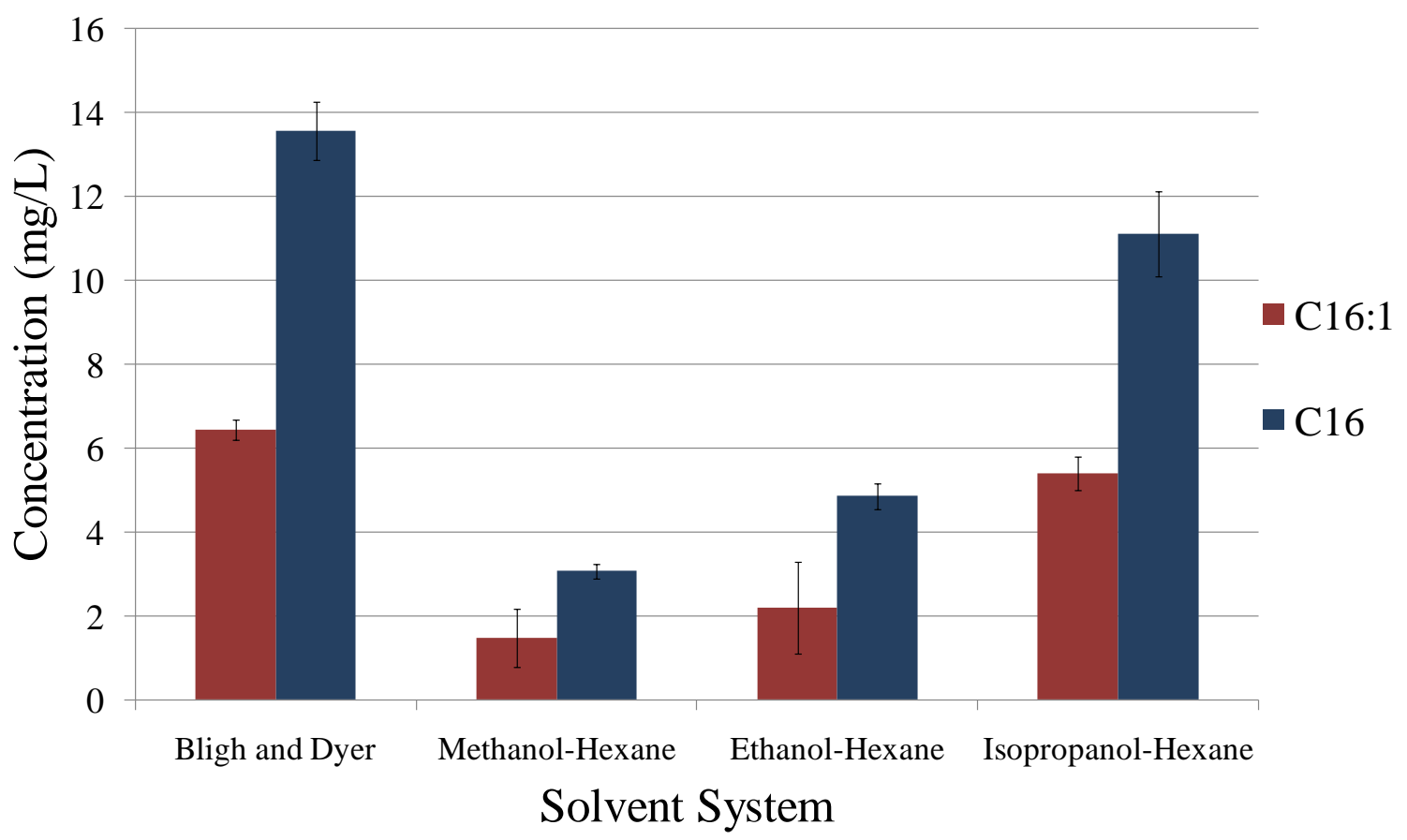

Figure 26: Fatty acid methyl esters extracted by solvent system 


\section{Chapter 5: Conclusions}

\section{Experimental Findings}

These experiments provide some insights into the potential of wastewater algae to support fuel production. They also provide information about the practicality of using different solvents in a future industrial process to produce algae biofuel.

\section{Lipid Production Experiment}

This set of experiments led to conclusions about the production rates of algal fatty acids and the types of fatty acids produced in batch cultures by algae growing with a municipal wastewater medium. It also led to some conclusions about the analytical methods currently used to study algal lipid production.

\section{Lipid Production}

The highest average rate of lipid production occurred during the period of rapid growth between Day 11 and Day 13. This peak production rate of lipids, $4.40 \mathrm{~g} / \mathrm{m}^{2} /$ day, determined by the chromatographic method, corresponds with approximately 1,900 gallons of biodiesel per acre per year. This figure is based on an assumed algae fuel density of $0.89 \mathrm{~g} / \mathrm{mL}$ and on an assumed 365 days per year of pond operation. It compares favorably to the results of a similar experiment conducted using dairy wastewater in which a biodiesel productivity of 1,200 gallons per acre per 365-day year was projected (Woertz, Feffer, Lundquist, \& Nelson, 2009). The average growth rate of 
$0.97 \mathrm{~g} / \mathrm{m}^{2} /$ day observed over the entire growth phase of the culture, from Day 4 to Day 17 , corresponds to approximately 420 gallons of biodiesel per acre per year.

These production values indicate that wastewater algae are capable of supplying enough oil to support industrial fuel production. The areal productivity observed in this study is much greater than values typically quoted for conventional biodiesel feedstocks such as palm and soy (Chisti, Biodiesel from microalgae, 2007). The non-ideal conditions of the present study (described below) suggest that the potential of algae as a biofuel feedstock may be even greater than reported at present (Chisti, 2007), (Woertz, Feffer, Lundquist, \& Nelson, 2009).

It is likely that even higher triglyceride productivities are possible with wastewater algae grown under improved conditions. For example, this experiment was conducted between March and April. The average daily high temperature of approximately $18{ }^{\circ} \mathrm{C}$ may be lower than ideal. Other studies have shown that biomass growth rates increase up to 37 ${ }^{\circ} \mathrm{C}$ (Sterner \& Grover, 1998), (Tedesco \& Duerr, 1989). With a depth of approximately $20 \mathrm{~cm}$, the algae culture was shaded by the 0.75 meter high walls of the pond. Studies on the effects of light on algae growth and lipid production indicate that triglyceride productivities in some species increase up to and beyond light intensities which may be expected in an outdoor algae pond (Tedesco \& Duerr, 1989). A culture set up in similar conditions would probably be more productive if shading was limited and the temperature was elevated. 


\section{Lipid Composition}

The most common fatty acids observed in the batch-mode pond were 16 and 18-carbon, saturated and monounsaturated fatty acids. These fatty acids are very similar in structure to those produced by more conventional oil crops such as soy and palm, which are well suited for liquid fuel production (Cruz, 1997).

The monounsaturated 18-carbon fatty acid observed in the algal culture is commonly referred to as oleic acid. The saturated 16-carbon fatty acid observed in the culture is commonly known as palmitic acid. Together, oleic acid and palmitic acid also constitute the bulk of palm oil. Soybean oil is primarily made up of a variety of unsaturated, unbranched eighteen-carbon fatty acids (Cruz, 1997). The similarity of the oils produced in the batch-mode algae pond to those of conventional oil crops suggests that a fuel product derived from wastewater algae might be characteristically similar to commercially available biodiesel fuel.

The tendency of the batch-mode culture to produce shorter fatty acid chains toward the end of the growth cycle has implications from a fuel production standpoint. This tendency in a fast-growing crop such as algae may make it possible for producers to have some control over the types of fatty acids grown in their systems. By manipulating the mean cell residence time of an algae pond, it may be possible to favor either longer or shorter carbon chains in the fatty acids produced. Batch-mode systems may be harvested at a certain time to target specific fatty acids. Semi-batch and continuous systems might be operated at different residence times to accomplish the same goal. 


\section{Discussion of Analytical Methods}

Lipid concentrations were determined by two different methods throughout the lipid production experiment. Much higher fatty acid contents were measured using the chromatographic method compared to the gravimetric method. However, the observed trends in lipid production were similar for both methods.

Due to the highly sensitive nature of gas chromatography and the low-temperature conditions in which the chromatographic samples were prepared, it is likely that the results of the chromatographic testing are more accurate than those of the gravimetric testing. Unlike the extractions for the chromatographic method, the gravimetric method included a heating step, which is a potential cause of the lower lipid concentrations compared with the chromatographic method. The heating of the gravimetric samples to a relatively high temperature $\left(105^{\circ} \mathrm{C}\right.$ for 1 hour $)$ may have caused some of the fatty acids to vaporize, although this temperature is not uncommon in Bligh and Dyer-based procedures.

The observation of a discrepancy between the gravimetric and chromatographic methods of oil determination is an important outcome of the current research. The lower temperature processing of the chromatographic samples and the analytical accuracy of mass spectrometry suggest that the chromatographic method provides a more realistic measure of the oil content of a sample. However, more research is needed to refine these methods and reconcile the discrepancy. 


\section{Extraction Procedure Experiment}

This thesis research produced results regarding the ability of several alcohol-based solvent systems to remove lipophilic mass from algae cells. It also provided new insight into the masses of triglycerides that may be extracted using each of the four solvent systems tested.

\section{Extractable Mass}

Of the four solvent systems examined, the methanol-water-chloroform (Bligh and Dyer) extracted the most mass from the algae samples, followed by methanol-hexane, ethanolhexane and isopropanol-hexane. The mass extracted increased with increasing solvent polarity in the alcohol-hexane solvent systems.

Chloroform is very nonpolar compared to the methanol-water mixture against which it is partitioned in the Bligh and Dyer extraction. Similarly, hexane is very nonpolar compared to methanol and so it attracts algal triglycerides strongly. Because isopropanol and hexane do not have as large a difference in polarities, the isopropanol system did not extract as much material from the algae as the methanol system.

\section{Fatty Acid Affinity}

The mass of fatty acids extracted by each solvent system, determined by chromatography, differed from the total mass of lipids by the gravimetric method. The Bligh and Dyer extraction removed the most fatty acids. However, among the alcohol-based extractions, the isopropanol system removed the most fatty acids ( $83 \%$ of Bligh and Dyer) and the methanol system removed the least (23\% of Bligh and Dyer). 
The alcohol-hexane extractions included no sonication step, while the Bligh and Dyer extraction did. The extractions, therefore, tested not only the solvents' ability to partition triglycerides efficiently, but also their ability to remove triglycerides from undisrupted algae cells. Methanol is known to be a more effective destructor of cell walls better than ethanol or isopropanol (Henriques, Silva, \& Rocha, 2007).

Although the isopropanol solvent system has a lower partition coefficient for triglycerides than the methanol solvent system, the isopropanol was able to remove more triglycerides from the algae because of its relatively high affinity for triglycerides.

\section{Additional Study}

The current research has provided several insights into the potential production and processing of algae-based biofuels. The present studies have also underlined the need to pursue several related topics of research.

\section{Algae Oil Production}

The present study has provided confirming evidence that algae can produce a greater quantity of oil than any conventional oil crop. However, the short-term nature and nonideal growth conditions of the current research leave unknown the upper limit of algae oil production rates. A similar study, performed in a warmer, sunnier climate over several years would help to answer remaining questions.

Carbon dioxide was used in the current study to control the $\mathrm{pH}$ of the algae pond. In an industrial system, carbon dioxide addition is expected to increase growth rates, while 
temporarily mitigating carbon dioxide emissions. A comprehensive investigation of the quantities and types of lipids produced in a pond with carbon dioxide addition compared with a pond without carbon dioxide addition would be useful. This would provide insight into whether carbon dioxide addition affects the lipid production rates and types of lipids grown in wastewater algae.

Additional study can also be targeted at assessing the level of control that can be achieved over the types of oils produced by manipulating the residence time of a pond system. A study comparing the residence time of a continuous-mode algae production system to the types of fatty acids produced would be valuable. This would provide new insights into the how algae ponds for fuel production should be set up.

\section{Algae and Fuel Processing}

The current study has also pointed to several potential areas of investigation regarding algae and fuel processing techniques.

This research only compared four different extraction procedures. There is a wide variety of solvents used in industrial extractions. In addition to the four combination studied in this research, a range of different combinations of solvents should be evaluated for use on microalgae.

Beyond the solvent systems tested in the present research, other extraction techniques are good candidates for further inquiry. Heated solvents are sometimes used in industrial facilities to improve rates of extraction. A simple study of the extractive capacity of a 
heated solvent compared to a solvent at ambient temperature would illustrate whether this is a worthwhile topic of investigation.

The use of different extraction technologies is also a topic of interest. Supercritical carbon dioxide extraction, although relatively expensive, may be a viable alternative to organic solvent extraction. Physical extraction methods, such as three-phase centrifugation may also prove to be good methods of producing biodiesel from algae on a large scale.

\section{Algae Oil Analysis}

All of the samples processed during the current research were analyzed by exhaustive laboratory methods. Although this was certainly useful in the laboratory setting, the level of reproducibility of these methods on an industrial scale is yet untested. The samples in these experiments were frozen under nitrogen and analyzed after their initial collected. It is likely that the freezing of algal cells is destructive to cell walls. A comparison of frozen and fresh analytes from the same sample would answer questions about the effects of freezing and thawing on the oils extracted.

The present research suggests that $\mathrm{C} 16$ and $\mathrm{C} 18$ fatty acids are most commonly produced by algae grown on wastewater. The chromatographic program used to analyze the samples was developed to investigate all possible fatty acids that may have been produced by the algae. A shorter chromatographic program would speed analysis.

The discrepancy between the results of the gravimetric and chromatographic methods of lipid determination should be investigated. Using the same temperature to vaporize 
solvents from samples prepared for both lipid determination procedures would help to reveal whether or not the $105^{\circ} \mathrm{C}$ heating step is responsible for the discrepancy. Other analysts have heated samples for both procedures to $50{ }^{\circ} \mathrm{C}$ (DeLuca, Charity. pers.

comm., 2009). The use of a vacuum oven instead of a nitrogen-sparged desiccator may also help to obtain consistent results (DeLuca, Charity. pers. comm., 2009). 


\section{References}

Andrich, G., Nesti, U., Venturi, F., Zinnai, A., \& Fiorentini, R. (2005). Supercritical fluid extraction of bioactive lipids from the microalga Nannochloropsis sp. European Journal of Lipid Science and Technology, pp. 381-386.

Asenjo, J. A. (1990). Cell disruption and removal of insolubles. In D. Pyle, Separations for Biotechnology 2 (pp. 11-20). London: Elsevier Applied Science.

B. Greg Mitchell, S. I. (2009). Algae as a Biofuel Feedstock. Federal Laboratory Consortium Far West and Mid-Continent Regional Meeting. Bulingame, California.

Bailey, P. S. (2000). Organic Chemistry. Upper Saddle River, NJ: Prentice-Hall, Inc.

Bannon, C. D., Craske, J. D., Felder, D. L., Garland, I. J., \& Norman, L. M. (1987). Analysis of fatty acid methyl esters with high accuracy and reliability. Journal of Chromatography, 231-241.

Belay, A. pers. comm. (2009, October). San Diego, California: Earthrise Nutritionals, LLC.

Benemann, J. R. (2008). Open Ponds and Closed Photobioreactors - Comparative Economics. 5th Annual World Congress on Industrial Biotechnology \& Bioprocessing. Chicago.

Benemann, J. R. (2008). Overview: Algae to Biofuels. NREL-AFOSR Workshop, Algal Oil for Jet Fuel Production. Arlington.

Benemann, J., Koopman, B., Weissman, J., Eisenberg, D., \& Goebel, R. (1980).

Development of Microalgae Harvesting and High-Rate Pond Technologies in California. Algae Biomass , 457-495.

Bligh, E., \& Dyer, W. (1959). A Rapid Method For Total Lipid Extraction and Purification. Canadian Journal of Biochemistry and Physiology, 911-917. 
Chaplin, M. (2004, December 20). London South Bank University. Retrieved July 9 , 2009, from Ultrasonic cell disruption:

http://www.lsbu.ac.uk/biology/enztech/ultrasonic.html

Chaumont, D. (1993). Biotechnology of algal biomass production: a review of systems for outdoor mass culture. Journal of Applied Phycology, 593-604.

Chisti, Y. (2007). Biodiesel from microalgae. Biotechnology Advances , 294-306.

Chisti, Y., \& Moo-Young, M. (1986). Disruption of microbial cells for intracellular products. Enzyme and Microbial Technology, 194-204.

Cohen, A. (1999). Chemicals from Microalgae. New York: CRC Press.

Cruz, P. S. (1997). Aquaculture Feed and Fertilizer Resource Atlas of the Philippines. Rome: Food and Agriculture Organization of the United Nations.

DeLuca, Charity. pers. comm. (2009, November 24). Livefuels Inc. San Carlos, California.

Dodd, J. (1981). Patent No. 4255261, Separation drum for belt filter with suction recovery of solids. United States.

Doucha, J., \& Livansky, K. (2008). Influence of processing parameters on disintegration of Chlorella cells in various types of homogenizers. Biotechnological Products and Process Engineering , 431-440.

Eder, K. (1995). Review: Gas chromatographic analysis of fatty acid methyl esters. Journal of Chromatography, 113-131.

Eder, K., Reichlmayr-Lais, A., \& Kirchgessner, M. (1991). Gas chromatographic analysis of fatty acid methyl esters: avoiding discrimination by programmed temperature vaporizing injection. Journal of Chromatography, 265-272.

Energy Information Administration. (2007). Annual Energy Outlook 2007. Washington, D.C: United States Department of Energy. 
Energy Information Administration. (2009). Monthly Energy Review June 2009. Washington, D.C.: United States Department of Energy.

Energy Information Administration. (2009). Renewable Energy Annual 2007.

Washington, D.C.: United States Department of Energy.

Enssani, E. (1987). Fundamental Parameters in Extraction of Lipids from WastewaterGrown Microalgal Biomass, Ph.D. Dissertation. Berkeley, California: University of California, Berkeley.

Feffer, A. S. (2007). The Effects of CO2 Addition on Algae Wastewater Treatment, M.S. Thesis. San Luis Obispo, California: Department of Civil and Environmental Engineering, California Polytechnic State University.

Food and Agricultural Organization of the United Nations. (2006). Livestock's Long Shadow: Environmental Issues and Options. Rome: United Nations.

Gardner, B., \& Tyner, W. (2007). Explorations in Biofuels Economics, Policy, and History. Journal of Agricultural \& Food Industrial Organization , pp. 1-6.

Gerpen, J. V. (2005). Biodiesel processing and production. Fuel Processing Technology, 1097-1107.

Greenberg, A. E. (1995). Standard Methods for the Examination of Water and Wastewater, 19th Edition. Washington, D.C.: American Public Health Association.

Gunstone, F. (1996). Fatty Acid and Lipid Chemistry. New York: Springer-Verlag.

Haas, M. J. (2005). Improving the economics of biodiesel production through the use of low value lipids as feedstocks: vegetable oil soapstock. Fuel Processing Technology, 1087-1096.

Hara, A., \& Radin, N. S. (1978). Lipid Extraction of Tissues with a Low Toxicity Solvent. Analytical Biochemistry, 420-426. 
Hayakawa, K., Tsujimura, S., Napolitano, G. E., Nakano, S.-i., Kumagai, M., Nakajima, T., et al. (2002). Fatty acid composition as an indicator of physiological condition of the cyanobacterium Microcystis aeruginosa. Limnology, 29-35.

Hejazi, M. A. (2004). Milking of Microalgae: Production and selective extraction of $\beta$ carotene in two-phase bioreactors. Wageningen: Wageningen University and Research Centre.

Henriques, M., Silva, A., \& Rocha, J. (2007). Extraction and quantification of pigments from a marine microalga: a simple and reproducible method. In A. Mendez-Vilas, Communicating Current Research and Educational Topics and Trends in Applied Microbiolog; Vol. 2 (pp. 586-593). Badajoz: Formatex.

Hu, Q., Sommerfeld, M., Jarvis, E., Ghirardi, M., Posewitz, M., Seibert, M., et al. (2008). Microalgal triacylglycerols as feedstocks for biofuel production: perspectives and advances. The Plant Journal , 632-639.

Intergovernmental Panel on Climate Change. (2008). Climate Change 2007: Synthesis Report. Geneva: Intergovernmental Panel on Climate Change.

International Programme on Chemical Safety. (2001). Chemical Safety Information for Intergovernmental Organizations. Retrieved October 11, 2009, from Dibutyl Sebacate: http://www.inchem.org/documents/icsc/icsc/eics1349.htm

IUPAC, Commision on the Nomenclature of Organic Chemistry. (1979). Nomenclature of Organic Chemistry. Sections A, B, C, D,E, F and H. Oxford: Pergamon Press.

Joshi, J. B., Elias, C. B., \& Patole, M. S. (1996). Role of hydrodynamic shear in the cultivation of animal, plant and microbial cells. The Biochemical Engineering Journal, 121-141.

Karmee, S. K., Mahesh, P., Ravi, R., \& Chadha, A. (2004). Kinetic Study of the BaseCatalyzed Transesterification of Monoglycerides from Pongamia Oil. Journal of the American Oil Chemist's Society, 425-430. 
Lavens, P., \& Sorgeloos, P. (1996). Manual on the Production and Use of Live Food for Aquaculture. Rome: Food and Agriculture Organization of the United Nations.

Lehr, Corinne. pers. comm. (2009, November 6). San Luis Obispo, California.

Lundquist, T. pers. comm. (2009, November 24). San Luis Obispo, California, United States.

Matt Gerhardt, B. a. (2009, October 8). The Nexus of Wastewater and Algae Biofuels. 2009 Algae Biomass Summit . San Diego, California, United States.

Metcalf and Eddy. (2003). Wastewater Engineering: Treatment and Reuse. New York: McGraw-Hill.

Michael Mussa. (2000). The Impact of High Oil Prices on the Global Economy. Washington, D.C.: The International Monetary Fund.

MWH. (2005). Water Treatment: Principles and Design. Hoboken, NJ: John Wiler and Sons, Inc.

Nagle, N., \& Lemke, P. (1990). Production of Methyl Ester Fuel from Microalgae. Applied Biochemistry and Biotechnology, pp. 355-361.

National Institute of Standards and Technology. (2006). National Institute of Standards and Technology. Retrieved October 14, 2009, from The NIST Reference on Constants, Units and Uncertainty: http://physics.nist.gov/cgi-bin/cuu/Value?auedm

National Institute of Standards and Technology. (2005, June 1). NIST MS Search Program 2.0. Gaithersburg, Maryland, United States.

Nelson, Y. pers. comm. (2009, April). San Luis Obispo, California, United States.

New Jersey Department of Health and Senior Services. (2001, June). Division of Epidemiology, Environmental and Occupational Health. Retrieved October 11, 2009, from Hazardous Substance Fact Sheet: DI-n-BUTYL PHTHALATE: http://nj.gov/health/eoh/rtkweb/documents/fs/0773.pdf 
Newton, T. (2009). The University of Southern Maine. Retrieved October 14, 2009, from $\mathrm{O}=$ CHem: http://usm.maine.edu/ newton/Chy251_253/Lectures/Solvents/Solvents.html NOAA National Climatic Data Center. (2009). Quality Controlled Local Clmatological Data. Washington, D.C., San Luis Obispo, California.

Ophardt, C. E. (2009). Fatty Acids. Retrieved 10 31, 2009, from Virtual Chembook: http://www.elmhurst.edu/ chm/vchembook/551 fattyacids.html

Orcutt, D. M., Parker, B. C., \& Lusby, W. R. (1986). Lipids in Blue-Green Algal Mats (Modern Stromatolites) from Antarctic Oasis Lakes. Journal of Phycology, 523-530.

Oswald, W. J. (1990). Advanced Integrated Wastewater Pond Systems. American Society of Civil Engineers Convention (pp. 73-81). San Francisco: American Society of Civil Engineers.

Oswald, W. J., \& Golueke, C. G. (1960). Biological Transformation of Solar Energy. Advances in Applied Microbiology, 223-262.

Oswald, W. J., Golueke, C. G., \& Gotass, H. B. (1957). Anaerobic Digestion of Algae. Applied Microbiology, 47-55.

Pimentel, D. (2003). Ethanol Fuels: Energy Balance, Economics, and Environmental Impacts are Negative. Natural Resources Research, 127-134.

Pimentel, D., \& Patzek, T. W. (2005). Ethanol Production Using Corn, Switchgrass, and Wood;. Natural Resources Research, 65-76.

Piorrek, M., Baasch, K.-H., \& Pohl, P. (1984). Biomass Production, Total Protein, Chlorophylls, Lipids and Fatty Acids of Freshwater Green and Blue-Green Algae Under Different Nitrogen Conditions. Phytochemistry, 207-216.

Prescott, G., Bamrick, J., Cawley, E., \& Jaques, W. (1978). How to Know the Freshwater Algae. New York: McGraw-Hill Science/Engineering/Math. 
Raemy, B. (2008). Scientific Challenges to Developing Marine Algal Biofuels - A View from the Field. Algal Commercialization Seminar - A UCSD initiative for Algal and Cyanobacterial Commercialization. San Diego.

Raymond, L. P. (1983). Aquatic Biomass as a Source of Fuels and Chemicals. Golden: Solar Energy Research Institute.

Rodolfi, L., Zittelli, G. C., Bassi, N., Padovani, G., Biondi, N., Bonini, G., et al. (2008). Microalgae for Oil: Strain Selection, Induction of Lipid Synthesis and Outdoor Mass Cultivation in a Low-Cost Photobioreactor. Biotechnology and Bioengineering , 100-112.

Rodolfi, L., Zittelli, G. C., Bassi, N., Padovani, G., Biondi, N., Bonini, G., et al. (2008). Microalgae for Oil: Strain Selection, Induction of Lipid Synthesis and Outdoor Mass Cultivation in a Low-Cost Photobioreactor. Biotechnology and Bioengineering , 100-112.

Rowlett, R. (2003, October 26). How Many? A Dictionary of Units of Measurement. Retrieved October 14, 2009, from CGS and MKS Units: http://www.unc.edu/ rowlett/units/cgsmks.html

Schwartz, M. (1972). Principles of Electrodynamics. New York: McGraw-Hill Incorporated.

Sheehan, J., Dunahay, T., Benemann, J., \& Roessler, P. (1998). A Look Back at the U.S. Department of Energy's Aquatic Species Program - Biodiesel from Algae. Golden: National Renewable Energy Laboratory.

Shuler, M. L. (2002). Bioprocess Engineering: Basic Concepts. Upper saddle Rive, NJ: Prentice-Hall, Inc.

Sikkema, J., Bont, J. A., \& Poolman, B. (1995). Mechanisms of Membrane Toxicity of Hydrocarbons. Microbiological Reviews , 201-222.

Sterner, R. W., \& Grover, J. P. (1998). Algal growth in warm temperature reservoirs: kinetic examination of nitrogen, temperature, light and other nutrients. Water Research, 3539-3548. 
Suen, Y., Hubbard, J. S., Holzer, G., \& Tornabene, T. G. (1987). Total Lipid Production of the Green Alga Nannochloropsis Sp.Q11 Under Different Nitrogen Regimes. Journal of Phycology , 289-296.

Tapanes, N. C., Aranda, D. A., Carneiro, J. W., \& Antunes, O. A. (2007).

Transesterification of Jatropha curcas oil glycerides: Theoretical and experimental studies of biodiesel reaction. Fuel , 2286-2295.

Tedesco, M. A., \& Duerr, E. O. (1989). Light, temperature and nitrogen starvation effects on the total lipid and fatty acid content and composition of Spirulina platensis UTEX 1928. Journal of Applied Phycology, 201-209.

The Paleontological Research Institution. (2009, June 29). Museum of the Earth. Retrieved June 29, 2009, from History of Oil: http://www.priweb.org/ed/pgws/history/history_home.html Tredici, M. R., \& Zittelli, G. C. (1998). Efficiency of Sunlight Utilization: Tubular Versus Flat Photobioreactors. Biotechnology and Bioengineering, 187-197.

U.S. Department of Energy. (2009). National Algal Biofuels Technology Roadmap. Washington, D.C.: U.S. Department of Energy.

Union of Concerned Scientists. (2009, May 13). Union of Concerned Scientists. Retrieved July 7, 2009, from Each Country's Share of CO2 Emissions: http://www.ucsusa.org/global_warming/science_and_impacts/science/each-countrysshare-of-co2.html

United States Energy Information Administration. (2009, June 6). Energy Information Administration. Retrieved September 7, 2009, from Petroleum Navigator: http://tonto.eia.doe.gov/dnav/pet/hist/mcrfpus2a.htm

United States Energy Information Administration. (2006). International Energy Annual. Washington, D.C.: United States Department of Energy. 
Vandanjon, L., Rossignol, N., Jaouen, P., Robert, J. M., \& Quemeneur, F. (1999). Effects of Shear on Two Microalgae Species. Contribution of Pumps and Valves in Tangential Flow Filtration Systems. Biotechnology and Bioengineering , 1-9.

Volmer, M., Meiborg, G., \& Muskiet, F. A. (1988). Simultaneous capillary gas chromatographic profiling of medium- and long-chain fatty acid methyl esters with split injection. Journal of Chromatography, 385-394.

Weissman, J. C., Goebel, R. P., \& Benemann, J. R. (1998). Photobioreactor Design:

Mixing, Carbon Utilization and Oxygen Accumulation. Biotechnology and

Bioengineering , 336-344.

Woertz, I. (2007). Lipid Productivity of Algae Grown on Dairy Wastewater as a Possible Feedstockfor Biodiesel. San Luis Obispo: Cal Poly.

Woertz, I., Feffer, A., Lundquist, T., \& Nelson, Y. (2009). Algae Grown on Dairy and Municipal Wastewater for Simultaneous Nutrient Removal and Lipid Production for Biofuel Feedstock. Journal of Environmental Engineering , 1115-1122.

Zhu, L., Zong, M., \& H.Wu. (2008). Efficient lipid production with Trichosporon fermentans and its use for biodiesel preparation. Bioresource Technology, 7881-7885. 


\section{Appendices}

\section{Appendix A: Lipid Production Experiment Data}

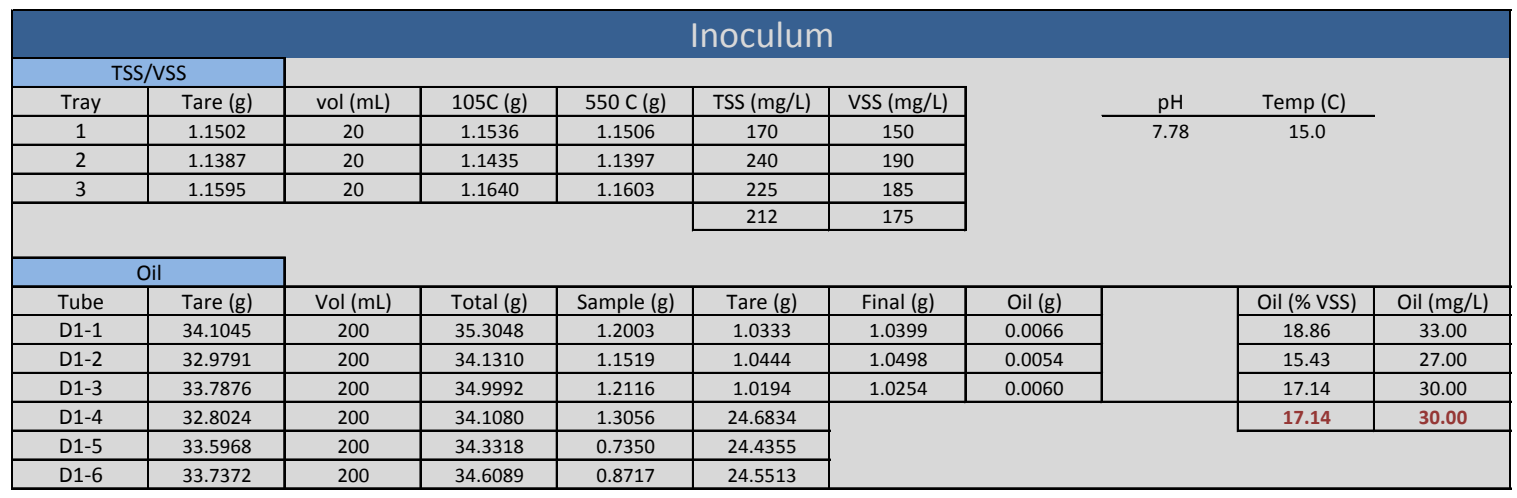

\begin{tabular}{|c|c|c|c|c|c|c|c|c|c|c|}
\hline \multicolumn{11}{|c|}{ Substrate } \\
\hline \multicolumn{2}{|c|}{ TSS/VSS } & & & & & & & \multirow{3}{*}{$\frac{\mathrm{pH}}{7.70}$} & \multirow[b]{2}{*}{ Temp (C) } & \\
\hline Tray & Tare (g) & $\mathrm{vol}(\mathrm{mL})$ & $105 \mathrm{C}(\mathrm{g})$ & $550 \mathrm{C}(\mathrm{g})$ & TSS (mg/L) & $\mathrm{VSS}(\mathrm{mg} / \mathrm{L})$ & & & & \\
\hline 1 & 1.1324 & 20 & 1.1340 & 1.1326 & 80 & 70 & & & \multirow[t]{4}{*}{20.0} & \\
\hline 2 & 1.1206 & 20 & 1.1222 & 1.1208 & 80 & 70 & & \multirow[t]{4}{*}{ 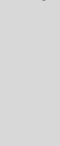 } & & \\
\hline 3 & 1.1624 & 20 & 1.1640 & 1.1625 & 80 & 75 & & & & \\
\hline & & & & 80 & 72 & & & & \\
\hline \multicolumn{2}{|c|}{ Oil } & & & & & & & & \multirow{2}{*}{ Oil (\% VSS) } & \multirow[b]{2}{*}{ Oil $(\mathrm{mg} / \mathrm{L})$} \\
\hline Tube & Tare (g) & $\mathrm{Vol}(\mathrm{mL})$ & Total (g) & Sample (g) & Tare (g) & Final (g) & Oil (g) & \multirow{4}{*}{. } & & \\
\hline D2-1 & 35.4402 & 200 & 35.5534 & 0.1132 & 1.0379 & 1.0401 & 0.0022 & & 15.35 & 11.00 \\
\hline D2-2 & 32.4757 & 200 & 32.5382 & 0.0625 & 1.0328 & 1.0350 & 0.0022 & & 15.35 & 11.00 \\
\hline D2-3 & 32.6524 & 200 & 32.7451 & 0.0927 & 1.0206 & 1.0226 & 0.0020 & & 13.95 & 10.00 \\
\hline D2-4 & 34.8513 & 200 & 35.0316 & 0.1803 & 24.6834 & & & & 14.88 & 10.67 \\
\hline $\mathrm{D} 2-5$ & 33.0543 & 200 & 33.1543 & 0.1000 & 24.4355 & & & & & \\
\hline D2-6 & 32.7613 & 200 & 32.9033 & 0.1420 & 24.5513 & & & & & \\
\hline
\end{tabular}

\begin{tabular}{|c|c|c|c|c|c|c|c|c|c|c|}
\hline \multicolumn{11}{|c|}{ Day 1} \\
\hline \multicolumn{2}{|c|}{ TSS/VSS } & & & & & & & \multirow[b]{2}{*}{$\mathrm{pH}$} & \multirow[b]{2}{*}{ Temp (C) } & \\
\hline Tray & Tare $(\mathrm{g})$ & vol $(\mathrm{mL})$ & $105 \mathrm{C}(\mathrm{g})$ & $550 \mathrm{C}(\mathrm{g})$ & TSS (mg/L) & VSS (mg/L) & & & & \\
\hline 1 & 1.1425 & 20 & 1.1450 & 1.1428 & 125 & 110 & & \multirow[t]{5}{*}{7.98} & \multirow[t]{5}{*}{16.8} & \\
\hline 2 & 1.1477 & 20 & 1.1498 & 1.1478 & 105 & 100 & & & & \\
\hline 3 & 1.1694 & 20 & 1.1718 & 1.1696 & 120 & 110 & & & & \\
\hline & & & & & 117 & 107 & & & & \\
\hline \multicolumn{2}{|c|}{ Oil } & & & & & & & & & \\
\hline Tube & Tare $(\mathrm{g})$ & Vol $(\mathrm{mL})$ & Total (g) & Sample (g) & Tare $(\mathrm{g})$ & Final $(\mathrm{g})$ & Oil (g) & & Oil (\% VSS) & Oil (mg/L) \\
\hline D1-1 & 36.2231 & 200 & 36.4719 & 0.2488 & 1.0434 & 1.0477 & 0.0043 & & 20.16 & 21.5 \\
\hline D1-2 & 36.5914 & 200 & 36.8289 & 0.2375 & 1.0129 & 1.0164 & 0.0035 & & 16.41 & 17.5 \\
\hline D1-3 & 36.2507 & 200 & 36.4749 & 0.2242 & 1.0438 & 1.0475 & 0.0037 & & 17.34 & 18.5 \\
\hline D1-4 & 33.4752 & 200 & 33.7014 & 0.2262 & 24.4136 & & & & \multirow[t]{3}{*}{17.97} & \multirow[t]{3}{*}{19.17} \\
\hline D1-5 & 35.3657 & 200 & 35.7311 & 0.3654 & 24.5889 & & & & & \\
\hline D1-6 & 36.2959 & 200 & 36.5055 & 0.2096 & 24.6448 & & & & & \\
\hline
\end{tabular}




\begin{tabular}{|c|c|c|c|c|c|c|c|c|c|c|}
\hline \multicolumn{11}{|c|}{ Day 2} \\
\hline \multicolumn{2}{|c|}{ TSS/VSS } & & & & & & & \multirow{3}{*}{$\begin{array}{c}\mathrm{pH} \\
8.01\end{array}$} & \multirow[b]{2}{*}{ Temp (C) } & \\
\hline Tray & Tare (g) & $\operatorname{vol}(\mathrm{mL})$ & $105 \mathrm{C}(\mathrm{g})$ & $550 \mathrm{C}(\mathrm{g})$ & TSS (mg/L) & VSS (mg/L) & & & & \\
\hline 1 & 1.1521 & 20 & 1.1543 & 1.1523 & 110 & 100 & & & \multirow[t]{4}{*}{14.0} & \\
\hline 2 & 1.1580 & 20 & 1.1594 & 1.1582 & 70 & 60 & & & & \\
\hline \multirow[t]{2}{*}{3} & 1.1493 & 20 & 1.1513 & 1.1495 & 100 & 90 & & & & \\
\hline & & & & & 93 & 83 & & & & \\
\hline \multicolumn{2}{|c|}{ Oil } & & & & & & & & & \\
\hline Tube & Tare (g) & Vol (mL) & Total (g) & Sample (g) & Tare (g) & Final (g) & Oil (g) & & Oil (\% VSS) & Oil (mg/L) \\
\hline D2-1 & 32.7890 & 200 & 33.0668 & 0.2778 & 1.0488 & 1.0514 & 0.0026 & & 15.60 & 13.00 \\
\hline D2-2 & 32.8267 & 200 & 33.0533 & 0.2266 & 1.0155 & 1.0179 & 0.0024 & & 14.40 & 12.00 \\
\hline D2-3 & 33.6622 & 200 & 33.8837 & 0.2215 & 1.0565 & 1.0594 & 0.0029 & & 17.40 & 14.50 \\
\hline D2-4 & 34.0916 & 200 & 34.2851 & 0.1935 & 24.6443 & & & & 15.80 & 13.17 \\
\hline D2-5 & 33.0282 & 200 & 33.2733 & 0.2451 & 24.5902 & & & & & \\
\hline D2-6 & 33.7908 & 200 & 34.1100 & 0.3192 & 24.4342 & & & & & \\
\hline
\end{tabular}

\begin{tabular}{|c|c|c|c|c|c|c|c|c|c|c|}
\hline \multicolumn{11}{|c|}{ Day 3} \\
\hline \multicolumn{2}{|c|}{ TSS/VSS } & & & & & & & \multirow{3}{*}{$\frac{\mathrm{pH}}{7.81}$} & \multirow[b]{2}{*}{ Temp (C) } & \\
\hline Tray & Tare (g) & $\operatorname{vol}(\mathrm{mL})$ & $105 C(g)$ & $550 \mathrm{C}(\mathrm{g})$ & TSS (mg/L) & $\operatorname{VSS}(\mathrm{mg} / \mathrm{L})$ & & & & \\
\hline 1 & 1.1597 & 20 & 1.1629 & 1.1598 & 160 & 155 & & & \multirow[t]{4}{*}{16.8} & \\
\hline 2 & 1.1596 & 20 & 1.1630 & 1.1601 & 170 & 145 & & & & \\
\hline \multirow[t]{2}{*}{3} & 1.1784 & 20 & 1.1815 & 1.1790 & 155 & 125 & & & & \\
\hline & & & & & 162 & 142 & & & & \\
\hline \multicolumn{2}{|c|}{ Oil } & & & & & & & & & \\
\hline Tube & Tare $(\mathrm{g})$ & Vol (mL) & Total (g) & Sample (g) & Tare (g) & Final (g) & Oil (g) & & Oil (\% VSS) & Oil $(\mathrm{mg} / \mathrm{L})$ \\
\hline D3-1 & 33.0103 & 200 & 33.2746 & 0.2643 & 1.0454 & 1.0497 & 0.0043 & & 15.18 & 21.50 \\
\hline D3-2 & 32.6260 & 200 & 32.9154 & 0.2894 & 1.0425 & 1.0459 & 0.0034 & & 12.00 & 17.00 \\
\hline D3-3 & 34.7453 & 200 & 35.0999 & 0.3546 & 1.0236 & 1.0273 & 0.0037 & & 13.06 & 18.50 \\
\hline D3-4 & 32.6781 & 200 & 32.9372 & 0.2591 & 24.4532 & & & & 13.41 & 19.00 \\
\hline D3-5 & 33.8009 & 200 & 34.0760 & 0.2751 & 24.4834 & & & & & \\
\hline D3-6 & 35.3806 & 200 & 35.6571 & 0.2765 & 24.6708 & & & & & \\
\hline
\end{tabular}

\begin{tabular}{|c|c|c|c|c|c|c|c|c|c|c|}
\hline \multicolumn{11}{|c|}{ Day 4} \\
\hline \multicolumn{2}{|c|}{ TSS/VSS } & & & & & & & \multirow[b]{2}{*}{$\mathrm{pH}$} & \multirow[b]{2}{*}{ Temp (C) } & \\
\hline Tray & Tare (g) & $\operatorname{vol}(\mathrm{mL})$ & $105 \mathrm{C}(\mathrm{g})$ & $550 \mathrm{C}(\mathrm{g})$ & $\mathrm{TSS}(\mathrm{mg} / \mathrm{L})$ & VSS ( $\mathrm{mg} / \mathrm{L})$ & & & & \\
\hline 1 & 1.1720 & 20 & 1.1752 & 1.1729 & 160 & 115 & & & \multirow[t]{4}{*}{20.7} & \\
\hline 2 & 1.1657 & 20 & 1.1690 & 1.1667 & 165 & 115 & & & & \\
\hline 3 & 1.1472 & 20 & 1.1509 & 1.1482 & 185 & 135 & & & & \\
\hline & & & & & 170 & 122 & & & & \\
\hline \multicolumn{2}{|c|}{ Oil } & & & & & & & & & \\
\hline Tube & Tare (g) & Vol (mL) & Total (g) & Sample (g) & Tare (g) & Final $(\mathrm{g})$ & Oil (g) & & Oil (\% VSS) & Oil $(\mathrm{mg} / \mathrm{L})$ \\
\hline D4-1 & 35.6265 & 200 & 35.9530 & 0.3265 & 1.0372 & 1.0414 & 0.0042 & & 17.26 & 21.00 \\
\hline D4-2 & 35.6872 & 200 & 36.0252 & 0.3380 & 1.0300 & 1.0345 & 0.0045 & & 18.49 & 22.50 \\
\hline D4-3 & 36.8000 & 200 & 37.2481 & 0.4481 & 1.0512 & 1.0550 & 0.0038 & & 15.62 & 19.00 \\
\hline D4-4 & 35.9335 & 200 & 36.2961 & 0.3626 & 24.6330 & & & & 17.12 & 20.83 \\
\hline D4-5 & 36.6662 & 200 & 37.1357 & 0.4695 & 24.4686 & & & & & \\
\hline D4-6 & 33.4900 & 200 & 33.7798 & 0.2898 & 24.6310 & & & & & \\
\hline
\end{tabular}

\begin{tabular}{|c|c|c|c|c|c|c|c|c|c|c|}
\hline \multicolumn{11}{|c|}{ Day 5} \\
\hline \multicolumn{2}{|c|}{ TSS/VSS } & & & & & & & \multirow{3}{*}{$\frac{\mathrm{pH}}{7.99}$} & \multirow[b]{2}{*}{ Temp (C) } & \\
\hline Tray & Tare (g) & $\operatorname{vol}(\mathrm{mL})$ & $105 \mathrm{C}(\mathrm{g})$ & $550 \mathrm{C}(\mathrm{g})$ & $\mathrm{TSS}(\mathrm{mg} / \mathrm{L})$ & $\operatorname{VSS}(\mathrm{mg} / \mathrm{L})$ & & & & \\
\hline 1 & 1.1716 & 20 & 1.1746 & 1.1718 & 150 & 140 & & & \multirow[t]{4}{*}{18.5} & \\
\hline 2 & 1.1566 & 20 & 1.1601 & 1.1572 & 175 & 145 & & & & \\
\hline 3 & 1.1621 & 20 & 1.1657 & 1.1629 & 180 & 140 & & & & \\
\hline & & & & & 168 & 142 & & & & \\
\hline \multicolumn{2}{|c|}{ Oil } & & & & & & & & & \\
\hline Tube & Tare (g) & Vol (mL) & Total (g) & Sample (g) & Tare (g) & Final (g) & Oil (g) & & Oil (\% VSS) & Oil (mg/L) \\
\hline D5-1 & 33.0729 & 200 & 33.3996 & $\begin{array}{l}0.3267 \\
\end{array}$ & 1.0353 & 1.0393 & 0.0040 & & 14.12 & 20.00 \\
\hline D5-2 & 32.7751 & 200 & 33.1047 & 0.3296 & 1.0122 & 1.0161 & 0.0039 & & 13.76 & 19.50 \\
\hline D5-3 & 34.0471 & 200 & 34.3985 & 0.3514 & 1.0251 & 1.0290 & 0.0039 & & 13.76 & 19.50 \\
\hline D5-4 & 32.7865 & 200 & 33.1795 & 0.3930 & 24.4660 & & & & 13.88 & 19.67 \\
\hline D5-5 & 33.7794 & 200 & 34.2319 & 0.4525 & 24.6231 & & & & & \\
\hline D5-6 & 33.5765 & 200 & 33.9161 & 0.3396 & 24.5117 & & & & & \\
\hline
\end{tabular}




\begin{tabular}{|c|c|c|c|c|c|c|c|c|c|c|}
\hline \multicolumn{11}{|c|}{ Day 6} \\
\hline \multicolumn{2}{|c|}{ TSS/VSS } & & & & & & & \multirow{3}{*}{$\begin{array}{c}\mathrm{pH} \\
8.42\end{array}$} & \multirow[b]{2}{*}{ Temp (C) } & \\
\hline Tray & Tare $(\mathrm{g})$ & $\operatorname{vol}(\mathrm{mL})$ & $105 \mathrm{C}(\mathrm{g})$ & $550 \mathrm{C}(\mathrm{g})$ & TSS (mg/L) & VSS (mg/L) & & & & \\
\hline 1 & 1.1592 & 20 & 1.1634 & 1.1599 & 210 & 175 & & & \multirow[t]{4}{*}{18.7} & \\
\hline 2 & 1.1524 & 20 & 1.1566 & 1.1533 & 210 & 165 & & & & \\
\hline 3 & 1.1692 & 20 & 1.1738 & 1.1701 & 230 & 185 & & & & \\
\hline & & & & & 217 & 175 & & & & \\
\hline \multicolumn{2}{|c|}{ Oil } & & & & & & & & & \\
\hline Tube & Tare $(\mathrm{g})$ & $\mathrm{Vol}(\mathrm{mL})$ & Total (g) & Sample (g) & Tare $(\mathrm{g})$ & Final $(\mathrm{g})$ & Oil (g) & & Oil (\% VSS) & Oil (mg/L) \\
\hline D6-1 & 35.4506 & 200 & 35.9464 & 0.4958 & 1.0104 & 1.0144 & 0.0040 & & 11.43 & 20.00 \\
\hline D6-2 & 33.1715 & 200 & 33.5697 & 0.3982 & 1.0126 & 1.0176 & 0.0050 & & 14.29 & 25.00 \\
\hline D6-3 & 36.1756 & 200 & 36.5988 & 0.4232 & 1.0385 & 1.0424 & 0.0039 & & 11.14 & 19.50 \\
\hline D6-4 & 33.0429 & 200 & 33.4347 & 0.3918 & 24.4171 & & & & 12.29 & 21.50 \\
\hline D6-5 & 35.6540 & 200 & 36.1308 & 0.4768 & 24.4823 & & & & & \\
\hline D6-6 & 34.1948 & 200 & 34.7707 & 0.5759 & 24.5167 & & & & & \\
\hline
\end{tabular}

\begin{tabular}{|c|c|c|c|c|c|c|c|c|c|c|}
\hline \multicolumn{11}{|c|}{ Day 7} \\
\hline \multicolumn{2}{|c|}{ TSS/VSS } & & & & & & & \multirow{3}{*}{$\frac{\mathrm{pH}}{8.28}$} & \multirow[b]{2}{*}{ Temp (C) } & \\
\hline Tray & Tare $(\mathrm{g})$ & $\operatorname{vol}(\mathrm{mL})$ & $105 C(\mathrm{~g})$ & $550 \mathrm{C}(\mathrm{g})$ & TSS (mg/L) & VSS (mg/L) & & & & \\
\hline 1 & 1.1501 & 20 & 1.1546 & 1.1510 & 225 & 180 & & & \multirow[t]{4}{*}{14.3} & \\
\hline 2 & 1.1634 & 20 & 1.1680 & 1.1644 & 230 & 180 & & & & \\
\hline \multirow[t]{2}{*}{3} & 1.1387 & 20 & 1.1429 & 1.1393 & 210 & 180 & & & & \\
\hline & & & & & 222 & 180 & & & & \\
\hline \multicolumn{2}{|c|}{ Oil } & & & & & & & & & \\
\hline Tube & Tare (g) & $\mathrm{Vol}(\mathrm{mL})$ & Total (g) & Sample (g) & Tare $(\mathrm{g})$ & Final $(\mathrm{g})$ & Oil (g) & & Oil (\% VSS) & Oil (mg/L) \\
\hline D7-1 & 34.0045 & 200 & 34.5481 & 0.5436 & 1.0320 & 1.0360 & 0.0040 & & 11.11 & 20.00 \\
\hline D7-2 & 32.7542 & 200 & 33.2719 & 0.5177 & 1.0255 & 1.0293 & 0.0038 & & 10.56 & 19.00 \\
\hline D7-3 & 33.0090 & 200 & 33.5482 & 0.5392 & 1.0198 & 1.0235 & 0.0037 & & 10.28 & 18.50 \\
\hline D7-4 & 36.2445 & 200 & 36.7622 & 0.5177 & 24.6445 & & & & 10.65 & 19.17 \\
\hline D7-5 & 32.9925 & 200 & 33.4984 & 0.5059 & 24.5665 & & & & & \\
\hline D7-6 & 33.6054 & 200 & 34.1094 & 0.5040 & 24.4927 & & & & & \\
\hline
\end{tabular}

\begin{tabular}{|c|c|c|c|c|c|c|c|c|c|c|}
\hline \multicolumn{2}{|c|}{ TSS/VSS } & & & & & & & \multirow{3}{*}{$\frac{\mathrm{pH}}{8.29}$} & \multirow[b]{2}{*}{ Temp (C) } & \\
\hline Tray & Tare (g) & $\operatorname{vol}(\mathrm{mL})$ & $105 \mathrm{C}(\mathrm{g})$ & $550 \mathrm{C}(\mathrm{g})$ & TSS (mg/L) & VSS ( $\mathrm{mg} / \mathrm{L})$ & & & & \\
\hline 1 & 1.1711 & 20 & 1.1759 & 1.1719 & 240 & 200 & & & \multirow[t]{4}{*}{14.7} & \\
\hline 2 & 1.1584 & 20 & 1.1635 & 1.1592 & 255 & 215 & & & & \\
\hline 3 & 1.1768 & 20 & 1.1820 & 1.1776 & 260 & 220 & & & & \\
\hline & & & & & 252 & 212 & & & & \\
\hline \multicolumn{2}{|c|}{ Oil } & & & & & & & & & \\
\hline Tube & Tare $(\mathrm{g})$ & $\mathrm{Vol}(\mathrm{mL})$ & Total (g) & Sample (g) & Tare $(\mathrm{g})$ & Final $(\mathrm{g})$ & Oil (g) & & Oil (\% VSS) & Oil (mg/L) \\
\hline D8-1 & 35.9491 & 200 & 36.5993 & 0.6502 & 1.0428 & 1.0468 & 0.0040 & & 9.45 & 20.00 \\
\hline D8-2 & 35.8409 & 200 & 36.5080 & 0.6671 & 1.0406 & 1.0445 & 0.0039 & & 9.21 & 19.50 \\
\hline D8-3 & 35.9735 & 200 & 36.5953 & 0.6218 & 1.0316 & 1.0357 & 0.0041 & & 9.69 & 20.50 \\
\hline D8-4 & 34.0938 & 200 & 34.6285 & 0.5347 & 24.5611 & & & & 9.45 & 20.00 \\
\hline D8-5 & 33.1417 & 200 & 33.6577 & 0.5160 & 24.4283 & & & & & \\
\hline D8-6 & 33.4145 & 200 & 34.0773 & 0.6628 & 24.5026 & & & & & \\
\hline
\end{tabular}

\begin{tabular}{|c|c|c|c|c|c|c|c|c|c|c|}
\hline \multicolumn{11}{|c|}{ Day 9} \\
\hline \multicolumn{2}{|c|}{ TSS/VSS } & & & & & & & \multirow{3}{*}{$\frac{\mathrm{pH}}{7.32}$} & \multirow[b]{2}{*}{ Temp (C) } & \\
\hline Tray & Tare $(\mathrm{g})$ & $\mathrm{vol}(\mathrm{mL})$ & $105 \mathrm{C}(\mathrm{g})$ & $550 \mathrm{C}(\mathrm{g})$ & TSS (mg/L) & VSS (mg/L) & & & & \\
\hline 1 & 1.1800 & 20 & 1.1852 & 1.1807 & 260 & 225 & & & \multirow[t]{4}{*}{14.1} & \\
\hline 2 & 1.1707 & 20 & 1.1767 & 1.1717 & 300 & 250 & & & & \\
\hline \multirow[t]{2}{*}{3} & 1.1623 & 20 & 1.1683 & 1.1630 & 300 & 265 & & & & \\
\hline & & & & & 287 & 247 & & & & \\
\hline \multicolumn{2}{|c|}{ Oil } & & & & & & & & \multirow{2}{*}{ Oil (\% VSS) } & \multirow[b]{2}{*}{ Oil (mg/L) } \\
\hline Tube & Tare $(\mathrm{g})$ & $\mathrm{Vol}(\mathrm{mL})$ & Total (g) & Sample (g) & Tare $(\mathrm{g})$ & Final $(\mathrm{g})$ & Oil (g) & \multirow{4}{*}{ 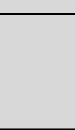 } & & \\
\hline D9-1 & 34.0571 & 200 & 34.5407 & 0.4836 & 0.9734 & 0.9777 & 0.0043 & & 11.94 & 21.50 \\
\hline D9-2 & 37.0778 & 200 & 37.9131 & 0.8353 & 0.9718 & 0.9758 & 0.0040 & & 11.11 & 20.00 \\
\hline D9-3 & 33.7463 & 200 & 34.4569 & 0.7106 & 0.9432 & 0.9468 & 0.0036 & & 10.00 & 18.00 \\
\hline D9-4 & 36.2175 & 200 & 36.8350 & 0.6175 & 24.1650 & & & & 11.02 & 19.83 \\
\hline D9-5 & 35.3504 & 200 & 36.0704 & 0.7200 & 24.1049 & & & & & \\
\hline D9-6 & 34.4307 & 200 & 35.0962 & 0.6655 & 24.9320 & & & & & \\
\hline
\end{tabular}




\begin{tabular}{|c|c|c|c|c|c|c|c|c|c|c|}
\hline \multicolumn{11}{|c|}{ Day 10} \\
\hline \multicolumn{2}{|c|}{ TSS/VSS } & & & & & & & \multirow[b]{2}{*}{$\mathrm{pH}$} & \multirow[b]{2}{*}{ Temp (C) } & \\
\hline Tray & Tare (g) & $\operatorname{vol}(\mathrm{mL})$ & $105 C(\mathrm{~g})$ & $550 \mathrm{C}(\mathrm{g})$ & TSS (mg/L) & VSS (mg/L) & & & & \\
\hline 1 & 1.1383 & 20 & 1.1449 & 1.1389 & 330 & 300 & & \multirow[t]{5}{*}{8.89} & \multirow[t]{4}{*}{14.0} & \\
\hline 2 & 1.1665 & 20 & 1.1733 & 1.1673 & 340 & 300 & & & & \\
\hline 3 & 1.1604 & 20 & 1.1672 & 1.1612 & 340 & 300 & & & & \\
\hline & & & & & 337 & 300 & & & & \\
\hline \multicolumn{2}{|c|}{ Oil } & & & & & & & & & \\
\hline Tube & Tare (g) & Vol (mL) & Total (g) & Sample (g) & Tare (g) & Final $(\mathrm{g})$ & Oil (g & & Oil (\% VSS) & Oil $(\mathrm{mg} / \mathrm{L})$ \\
\hline \multicolumn{11}{|l|}{ D10-1 } \\
\hline \multicolumn{11}{|l|}{ D10-2 } \\
\hline \multicolumn{11}{|l|}{ D10-3 } \\
\hline \multirow{2}{*}{\multicolumn{11}{|c|}{$\frac{D 10-4}{D 10-5}$}} \\
\hline & & & & & & & & & & \\
\hline D10-6 & & & & & & & & & & \\
\hline
\end{tabular}

\begin{tabular}{|c|c|c|c|c|c|c|c|c|c|c|}
\hline \multicolumn{11}{|c|}{ Day 11} \\
\hline \multicolumn{2}{|c|}{ TSS/VSS } & & & & & & & \multirow[b]{2}{*}{$\mathrm{pH}$} & \multirow[b]{2}{*}{ Temp (C) } & \\
\hline Tray & Tare (g) & $\operatorname{vol}(\mathrm{mL})$ & $105 \mathrm{C}(\mathrm{g})$ & $550 \mathrm{C}(\mathrm{g})$ & $\mathrm{TSS}(\mathrm{mg} / \mathrm{L})$ & $\operatorname{VSS}(\mathrm{mg} / \mathrm{L})$ & & & & \\
\hline 1 & 1.1723 & 20 & 1.1798 & 1.1736 & 375 & 310 & & \multirow{5}{*}{8.09} & \multirow[t]{4}{*}{15.0} & \\
\hline 2 & 1.1740 & 20 & 1.1817 & 1.1753 & 385 & 320 & & & & \\
\hline 3 & 1.1425 & 20 & 1.1504 & 1.1440 & 395 & 320 & & & & \\
\hline & & & & & 385 & 317 & & & & \\
\hline \multicolumn{2}{|c|}{ Oil } & & & & & & & & & \\
\hline Tube & Tare $(\mathrm{g})$ & Vol (mL) & Total (g) & Sample (g) & Tare (g) & Final $(\mathrm{g})$ & Oil (g) & & Oil (\% VSS) & Oil $(\mathrm{mg} / \mathrm{L})$ \\
\hline D11-1 & 33.6734 & 200 & 34.3410 & 0.6676 & 0.9504 & 0.9582 & 0.0078 & & 12.32 & 39.00 \\
\hline D11-2 & 33.2310 & 200 & 34.0773 & 0.8463 & 0.9548 & 0.9614 & 0.0066 & & 10.42 & 33.00 \\
\hline D11-3 & 33.4344 & 200 & 34.2542 & 0.8198 & 0.9662 & 0.9734 & 0.0072 & & 11.37 & 36.00 \\
\hline D11-4 & 33.6636 & 200 & 34.4055 & 0.7419 & 24.7188 & & & & 11.37 & 36.00 \\
\hline D11-5 & 33.1678 & 200 & 33.9479 & 0.7801 & 24.5434 & & & & & \\
\hline D11-6 & 35.7733 & 200 & 36.5217 & 0.7484 & 24.7197 & & & & & \\
\hline
\end{tabular}

\begin{tabular}{|c|c|c|c|c|c|c|c|c|c|c|}
\hline \multicolumn{11}{|c|}{ Day 12} \\
\hline \multicolumn{2}{|c|}{ TSS/VSS } & & & & & & & \multirow[b]{2}{*}{$\mathrm{pH}$} & \multirow[b]{2}{*}{ Temp (C) } & \\
\hline Tray & Tare (g) & $\operatorname{vol}(\mathrm{mL})$ & $105 \mathrm{C}(\mathrm{g})$ & $550 \mathrm{C}(\mathrm{g})$ & $\mathrm{TSS}(\mathrm{mg} / \mathrm{L})$ & VSS (mg/L) & & & & \\
\hline 1 & 1.1542 & 20 & 1.1623 & 1.1552 & 405 & 355 & & \multirow{5}{*}{8.68} & \multirow[t]{4}{*}{18.3} & \\
\hline 2 & 1.1604 & 20 & 1.1690 & 1.1614 & 430 & 380 & & & & \\
\hline 3 & 1.1710 & 20 & 1.1785 & 1.1722 & 375 & 315 & & & & \\
\hline & & & & & 403 & 350 & & & & \\
\hline \multicolumn{2}{|c|}{ Oil } & & & & & & & & & \\
\hline Tube & Tare (g) & Vol (mL) & Total (g) & Sample (g) & Tare (g) & Final $(\mathrm{g})$ & Oil (g) & & Oil (\% VSS) & Oil $(\mathrm{mg} / \mathrm{L})$ \\
\hline \multicolumn{11}{|l|}{ D12-1 } \\
\hline \multicolumn{11}{|l|}{ D12-2 } \\
\hline \multicolumn{11}{|l|}{ D12-3 } \\
\hline \multirow{2}{*}{\multicolumn{11}{|c|}{$\begin{array}{l}\text { D12-4 } \\
\text { D12-5 }\end{array}$}} \\
\hline & & & & & & & & & & \\
\hline D12-6 & & & & & & & & & & \\
\hline
\end{tabular}

\begin{tabular}{|c|c|c|c|c|c|c|c|c|c|c|}
\hline \multicolumn{11}{|c|}{ Day 13} \\
\hline \multicolumn{2}{|c|}{ TSS/VSS } & & & & & & & \multirow[b]{2}{*}{$\mathrm{pH}$} & \multirow[b]{2}{*}{ Temp (C) } & \\
\hline Tray & Tare (g) & $\operatorname{vol}(\mathrm{mL})$ & $105 \mathrm{C}(\mathrm{g})$ & $550 \mathrm{C}(\mathrm{g})$ & TSS (mg/L) & VSS ( $\mathrm{mg} / \mathrm{L})$ & & & & \\
\hline 1 & 1.1764 & 20 & 1.1852 & 1.1777 & 440 & 375 & & \multirow[t]{5}{*}{8.28} & \multirow[t]{4}{*}{18.9} & \\
\hline 2 & 1.1656 & 20 & 1.1744 & 1.1669 & 440 & 375 & & & & \\
\hline 3 & 1.1911 & 20 & 1.2003 & 1.1924 & 460 & 395 & & & & \\
\hline & & & & & 447 & 382 & & & & \\
\hline \multicolumn{2}{|c|}{ Oil } & & & & & & & & & \\
\hline Tube & Tare (g) & Vol (mL) & Total (g) & Sample (g) & Tare (g) & Final (g) & Oil (g) & & Oil (\% VSS) & Oil (mg/L) \\
\hline D13-1 & 33.1161 & 200 & 34.1115 & 0.9954 & 1.0308 & 1.0393 & 0.0085 & & 11.14 & 42.50 \\
\hline D13-2 & 36.0003 & 200 & 36.8712 & 0.8709 & 1.0208 & 1.0268 & 0.0060 & & 7.86 & 30.00 \\
\hline D13-3 & 33.0305 & 200 & 33.9141 & 0.8836 & 1.0306 & 1.0374 & 0.0068 & & 8.91 & 34.00 \\
\hline D13-4 & 33.9539 & 200 & 34.7039 & 0.7500 & 24.4724 & & & & 9.30 & 35.50 \\
\hline D13-5 & 35.7948 & 200 & 36.5896 & 0.7948 & 24.6857 & & & & & \\
\hline D13-6 & 33.4954 & 200 & 34.3603 & 0.8649 & 24.5709 & & & & & \\
\hline
\end{tabular}




\begin{tabular}{|c|c|c|c|c|c|c|c|c|c|c|}
\hline \multicolumn{11}{|c|}{ Day 14} \\
\hline \multicolumn{2}{|c|}{ TSS/VSS } & & & & & & & \multirow{3}{*}{$\mathrm{pH}$} & \multirow[b]{2}{*}{ Temp (C) } & \\
\hline Tray & Tare (g) & $\operatorname{vol}(\mathrm{mL})$ & $105 C(\mathrm{~g})$ & $550 \mathrm{C}(\mathrm{g})$ & TSS (mg/L) & VSS (mg/L) & & & & \\
\hline 1 & 1.1830 & 20 & 1.1925 & 1.1842 & 475 & 415 & & & \multirow[t]{4}{*}{16.2} & \\
\hline 2 & 1.1639 & 20 & 1.1739 & 1.1661 & 500 & 390 & & & & \\
\hline \multirow[t]{2}{*}{3} & 1.1561 & 20 & 1.1663 & 1.1576 & 510 & 435 & & & & \\
\hline & & & & & 495 & 413 & & & & \\
\hline \multicolumn{2}{|c|}{ Oil } & & & & & & & & & \\
\hline Tube & Tare (g) & Vol (mL) & Total (g) & Sample (g) & Tare (g) & Final (g) & Oil (g & & Oil (\% VSS) & Oil $(\mathrm{mg} / \mathrm{L})$ \\
\hline \multicolumn{11}{|l|}{ D14-1 } \\
\hline \multicolumn{11}{|l|}{ D14-2 } \\
\hline \multicolumn{11}{|l|}{ D14-3 } \\
\hline \multirow{2}{*}{\multicolumn{11}{|c|}{$\frac{D 14-4}{D 14-5}$}} \\
\hline & & & & & & & & & & \\
\hline \multicolumn{11}{|l|}{$\begin{array}{l}\text { D14-5 } \\
\text { D144-6 }\end{array}$} \\
\hline
\end{tabular}

\begin{tabular}{|c|c|c|c|c|c|c|c|c|c|c|}
\hline \multicolumn{11}{|c|}{ Day 15} \\
\hline \multicolumn{2}{|c|}{ TSS/VSS } & & & & & & & \multirow{3}{*}{$\frac{\mathrm{pH}}{7.21}$} & \multirow[b]{2}{*}{ Temp (C) } & \\
\hline Tray & Tare (g) & $\operatorname{vol}(\mathrm{mL})$ & $105 \mathrm{C}(\mathrm{g})$ & $550 \mathrm{C}(\mathrm{g})$ & $\mathrm{TSS}(\mathrm{mg} / \mathrm{L})$ & $\operatorname{VSS}(\mathrm{mg} / \mathrm{L})$ & & & & \\
\hline 1 & 1.1711 & 20 & 1.1811 & 1.1722 & 500 & 445 & & & \multirow{4}{*}{10.7} & \\
\hline 2 & 1.1473 & 20 & 1.1574 & 1.1481 & 505 & 465 & & & & \\
\hline \multirow[t]{2}{*}{3} & 1.1658 & 20 & 1.1758 & 1.1668 & 500 & 450 & & & & \\
\hline & & & & & 502 & 453 & & & & \\
\hline \multicolumn{2}{|c|}{ Oil } & & & & & & & & & \\
\hline Tube & Tare $(\mathrm{g})$ & Vol (mL) & Total (g) & Sample (g) & Tare (g) & Final $(\mathrm{g})$ & Oil (g) & & Oil (\% VSS) & Oil $(\mathrm{mg} / \mathrm{L})$ \\
\hline D15-1 & 35.4812 & 200 & 36.5470 & 1.0658 & 0.9621 & 0.9663 & 0.0042 & & 4.63 & 21.00 \\
\hline D15-2 & 35.5127 & 200 & 36.5799 & 1.0672 & 0.9686 & 0.9727 & 0.0041 & & 4.52 & 20.50 \\
\hline D15-3 & 35.0809 & 200 & 35.9961 & 0.9152 & 0.9728 & 0.9762 & 0.0034 & & 3.75 & 17.00 \\
\hline D15-4 & 35.6714 & 200 & 36.7494 & 1.0780 & 24.4806 & & & & 4.30 & 19.50 \\
\hline D15-5 & 33.4797 & 200 & 34.4642 & 0.9845 & 24.7938 & & & & & \\
\hline D15-6 & 35.2278 & 200 & 36.2507 & 1.0229 & 24.6881 & & & & & \\
\hline
\end{tabular}

\begin{tabular}{|c|c|c|c|c|c|c|c|c|c|c|}
\hline \multicolumn{11}{|c|}{ Day 16} \\
\hline \multicolumn{2}{|c|}{ TSS/VSS } & & & & & & & \multirow[b]{2}{*}{$\mathrm{pH}$} & \multirow[b]{2}{*}{ Temp (C) } & \\
\hline Tray & Tare (g) & $\operatorname{vol}(\mathrm{mL})$ & $105 \mathrm{C}(\mathrm{g})$ & $550 \mathrm{C}(\mathrm{g})$ & $\mathrm{TSS}(\mathrm{mg} / \mathrm{L})$ & VSS (mg/L) & & & & \\
\hline 1 & 1.1590 & 20 & 1.1696 & 1.1596 & 530 & 500 & & \multirow{5}{*}{8.71} & \multirow{4}{*}{17.1} & \\
\hline 2 & 1.1621 & 20 & 1.1731 & 1.1637 & 550 & 470 & & & & \\
\hline 3 & 1.1819 & 20 & 1.1940 & 1.1836 & 605 & 520 & & & & \\
\hline & & & & & 562 & 497 & & & & \\
\hline \multicolumn{2}{|c|}{ Oil } & & & & & & & & & \\
\hline Tube & Tare (g) & Vol (mL) & Total (g) & Sample (g) & Tare (g) & Final $(\mathrm{g})$ & Oil (g) & & Oil (\% VSS) & Oil $(\mathrm{mg} / \mathrm{L})$ \\
\hline \multicolumn{11}{|l|}{ D16-1 } \\
\hline \multicolumn{11}{|l|}{ D16-2 } \\
\hline \multicolumn{11}{|l|}{ D16-3 } \\
\hline \multirow{2}{*}{\multicolumn{11}{|c|}{$\begin{array}{l}\text { D16-4 } \\
\text { D16-5 }\end{array}$}} \\
\hline & & & & & & & & & & \\
\hline D16-6 & & & & & & & & & & \\
\hline
\end{tabular}

\begin{tabular}{|c|c|c|c|c|c|c|c|c|c|c|}
\hline \multicolumn{11}{|c|}{ Day 17} \\
\hline \multicolumn{2}{|c|}{ TSS/VSS } & & & & & & & \multirow{3}{*}{$\frac{\mathrm{pH}}{7.84}$} & \multirow[b]{2}{*}{ Temp (C) } & \\
\hline Tray & Tare (g) & $\operatorname{vol}(\mathrm{mL})$ & $105 \mathrm{C}(\mathrm{g})$ & $550 \mathrm{C}(\mathrm{g})$ & TSS (mg/L) & $\mathrm{VSS}(\mathrm{mg} / \mathrm{L})$ & & & & \\
\hline 1 & 1.1363 & 20 & 1.1471 & 1.1373 & 540 & 490 & & & \multirow[t]{4}{*}{15.3} & \\
\hline 2 & 1.1658 & 20 & 1.1789 & 1.1672 & 655 & 585 & & & & \\
\hline 3 & 1.1836 & 20 & 1.1954 & 1.1853 & 590 & 505 & & & & \\
\hline & & & & & 595 & 527 & & & & \\
\hline \multicolumn{2}{|c|}{ Oil } & & & & & & & & & \\
\hline Tube & Tare (g) & $\operatorname{Vol}(\mathrm{mL})$ & Total (g) & Sample (g) & Tare (g) & Final (g) & Oil (g) & & Oil (\% VSS) & Oil $(\mathrm{mg} / \mathrm{L})$ \\
\hline D17-1 & 33.3708 & 200 & 34.4244 & 1.0536 & 0.9567 & 0.9628 & 0.0061 & & 5.79 & 30.50 \\
\hline D17-2 & 35.6704 & 200 & 36.8529 & 1.1825 & 0.9475 & 0.9550 & 0.0075 & & 7.12 & 37.50 \\
\hline D17-3 & 33.8601 & 200 & 35.0365 & 1.1764 & 0.9535 & 0.9605 & 0.0070 & & 6.65 & 35.00 \\
\hline D17-4 & 34.0103 & 200 & 35.0867 & 1.0764 & 24.6547 & & & & 6.52 & 34.33 \\
\hline D17-5 & 32.7864 & 200 & 33.9398 & 1.1534 & 24.5832 & & & & & \\
\hline D17-6 & 35.9118 & 200 & 37.0634 & 1.1516 & 22.4820 & & & & & \\
\hline
\end{tabular}




\begin{tabular}{|c|c|c|c|c|c|c|c|c|c|c|}
\hline \multicolumn{11}{|c|}{ Day 22} \\
\hline \multicolumn{2}{|c|}{ TSS/VSS } & & & & & & & \multirow[b]{2}{*}{$\mathrm{pH}$} & \multirow[b]{2}{*}{ Temp (C) } & \\
\hline Tray & Tare (g) & $\operatorname{vol}(\mathrm{mL})$ & $105 C(\mathrm{~g})$ & $550 \mathrm{C}(\mathrm{g})$ & TSS (mg/L) & VSS (mg/L) & & & & \\
\hline 1 & 1.1623 & 20 & 1.1680 & 1.1627 & 285 & 265 & & \multirow[t]{5}{*}{7.42} & \multirow[t]{4}{*}{10.3} & \\
\hline 2 & 1.1725 & 20 & 1.1783 & 1.1729 & 290 & 270 & & & & \\
\hline 3 & 1.1679 & 20 & 1.1743 & 1.1691 & 320 & 260 & & & & \\
\hline & & & & & 298 & 265 & & & & \\
\hline \multicolumn{2}{|c|}{ Oil } & & & & & & & & & \\
\hline Tube & Tare (g) & Vol (mL) & Total (g) & Sample (g) & Tare (g) & Final $(\mathrm{g})$ & Oil (g & & Oil (\% VSS) & Oil $(\mathrm{mg} / \mathrm{L})$ \\
\hline \multicolumn{11}{|l|}{ D22-1 } \\
\hline \multicolumn{11}{|l|}{ D22-2 } \\
\hline \multicolumn{11}{|l|}{ D22-3 } \\
\hline \multirow{2}{*}{\multicolumn{11}{|c|}{$\frac{D 22-4}{D 22-5}$}} \\
\hline & & & & & & & & & & \\
\hline D22-6 & & & & & & & & & & \\
\hline
\end{tabular}

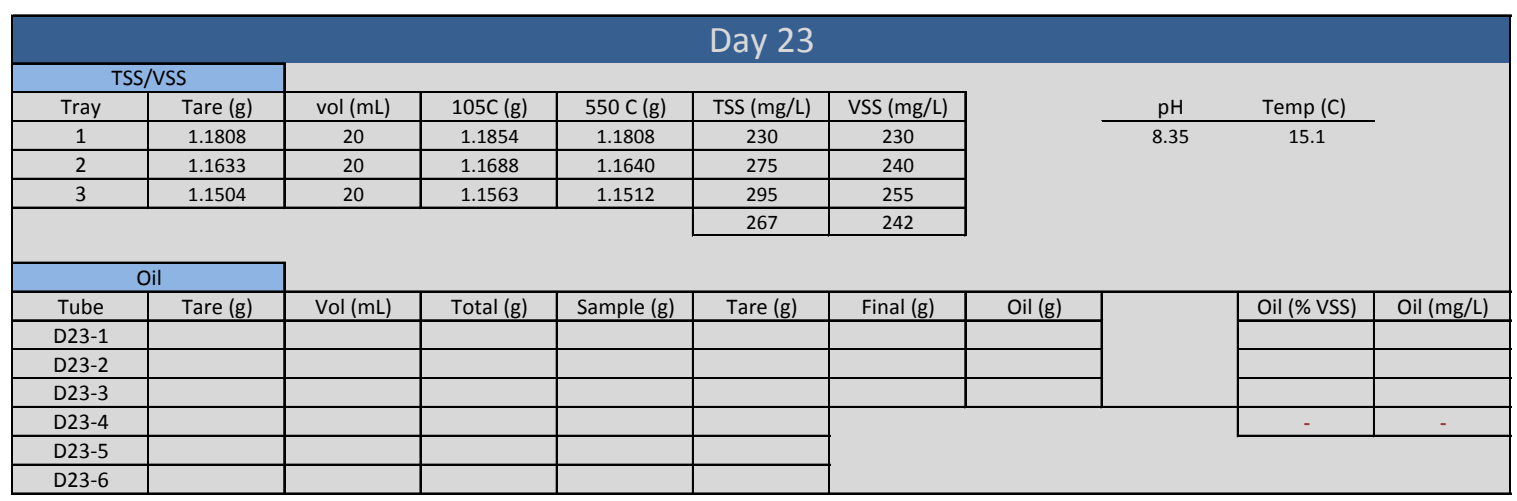

\begin{tabular}{|c|c|c|c|c|c|c|c|c|c|c|}
\hline \multicolumn{11}{|c|}{ Day 24} \\
\hline \multicolumn{2}{|c|}{ TSS/VSS } & & & & & & & \multirow{3}{*}{$\frac{\mathrm{pH}}{783}$} & \multirow[b]{2}{*}{ Temp (C) } & \\
\hline Tray & Tare (g) & vol (mL) & $105 \mathrm{C}(\mathrm{g})$ & $550 \mathrm{C}(\mathrm{g})$ & $\mathrm{TSS}(\mathrm{mg} / \mathrm{L})$ & $\operatorname{VSS}(\mathrm{mg} / \mathrm{L})$ & & & & \\
\hline 1 & 1.1918 & 20 & 1.1958 & 1.1919 & 200 & 195 & & & \multirow[t]{4}{*}{13.9} & \\
\hline 2 & 1.1478 & 20 & 1.1528 & 1.1481 & 250 & 235 & & & & \\
\hline \multirow[t]{2}{*}{3} & 1.1653 & 20 & 1.1701 & 1.1656 & 240 & 225 & & & & \\
\hline & & & & & 230 & 218 & & & & \\
\hline \multicolumn{2}{|c|}{ Oil } & & & & & & & & & \\
\hline Tube & Tare (g) & Vol (mL) & Total (g) & Sample (g) & Tare $(\mathrm{g})$ & Final $(\mathrm{g})$ & Oil (g) & & Oil (\% VSS) & Oil (mg/L) \\
\hline \multicolumn{11}{|l|}{ D24-1 } \\
\hline \multicolumn{11}{|l|}{ D24-2 } \\
\hline \multicolumn{11}{|l|}{ D24-3 } \\
\hline \multirow{2}{*}{\multicolumn{11}{|c|}{$\frac{\mathrm{D} 24-4}{\mathrm{D} 24-5}$}} \\
\hline & & & & & & & & & & \\
\hline \multicolumn{11}{|l|}{$\begin{array}{l}\text { D24-5 } \\
\text { D24-6 }\end{array}$} \\
\hline
\end{tabular}

\begin{tabular}{|c|c|c|c|c|c|c|c|c|c|c|}
\hline \multicolumn{11}{|c|}{ Day 25} \\
\hline \multicolumn{2}{|c|}{ TSS/VSS } & & & & & & & \multirow{3}{*}{$\frac{\mathrm{pH}}{7.76}$} & \multirow[b]{2}{*}{ Temp (C) } & \\
\hline Tray & Tare (g) & $\operatorname{vol}(\mathrm{mL})$ & $105 \mathrm{C}(\mathrm{g})$ & $550 \mathrm{C}(\mathrm{g})$ & $\mathrm{TSS}$ (mg/L) & $\operatorname{VSS}(\mathrm{mg} / \mathrm{L})$ & & & & \\
\hline 1 & 1.1802 & 20 & 1.1853 & 1.1804 & 255 & 245 & & & 13.0 & \\
\hline 2 & 1.1858 & 20 & 1.1903 & 1.1858 & 225 & 225 & & & & \\
\hline \multirow[t]{2}{*}{3} & 1.1854 & 20 & 1.1896 & 1.1857 & 210 & 195 & & & & \\
\hline & & & & & 230 & 222 & & & & \\
\hline \multicolumn{2}{|c|}{ Oil } & & & & & & & & & \\
\hline Tube & Tare (g) & Vol (mL) & Total (g) & Sample (g) & Tare (g) & Final (g) & Oil (g) & & Oil (\% VSS) & Oil (mg/L) \\
\hline \multicolumn{11}{|l|}{ D25-1 } \\
\hline \multicolumn{11}{|l|}{ D25-2 } \\
\hline \multicolumn{11}{|l|}{ D25-3 } \\
\hline \multirow{2}{*}{\multicolumn{11}{|c|}{$\frac{\mathrm{D} 25-4}{\mathrm{D} 25-5}$}} \\
\hline & & & & & & & & & & \\
\hline D25-6 & & & & & & & & & & \\
\hline
\end{tabular}




\section{Appendix B: Extraction Procedure Experiment Data}

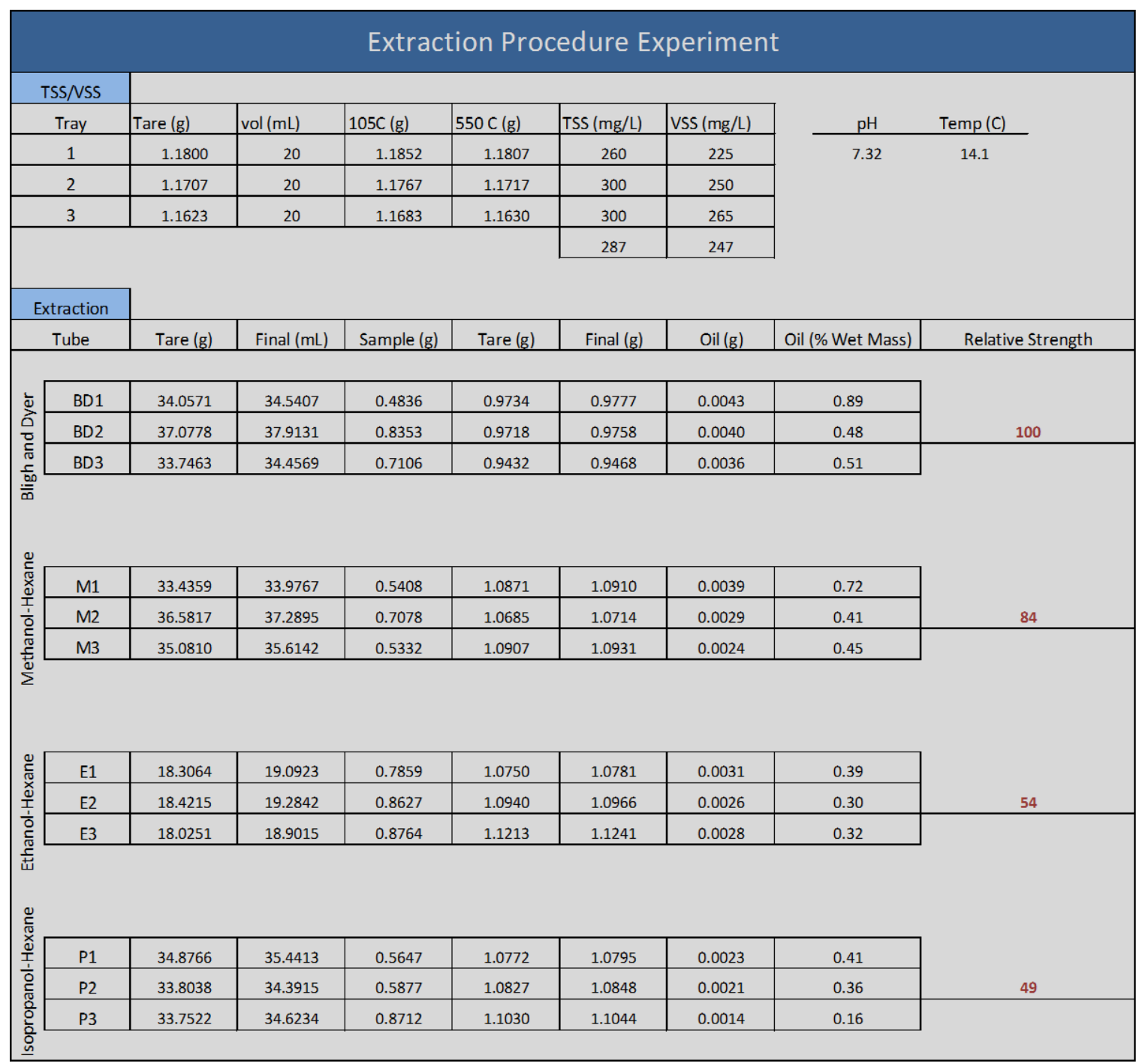




\section{Appendix C: Quality Control Results}

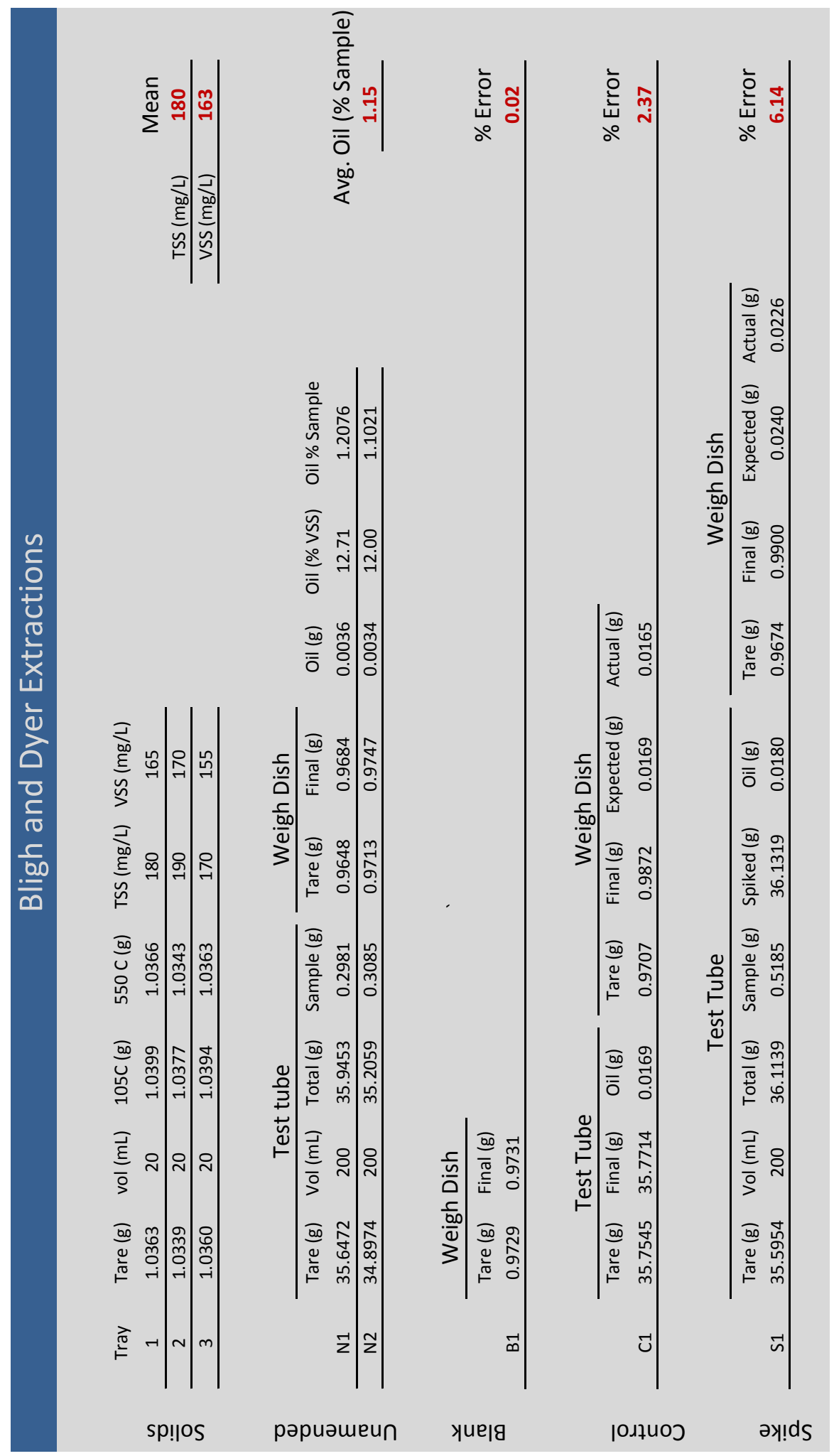




\section{Alcohol-Hexane Extractions}

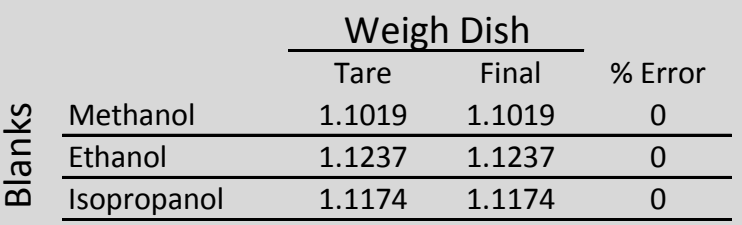

\begin{tabular}{|c|c|c|c|c|c|c|c|c|}
\hline & & & est Tub & & & eigh D & & \\
\hline & & Tare & Final & Oil & Tare & Final & Oil & $\%$ Error \\
\hline$\tilde{d}$ & Methanol & 23.9742 & 23.9818 & 0.0076 & 1.1157 & 1.1232 & 0.0075 & 1.32 \\
\hline$\frac{y}{\circ}$ & Ethanol & 23.9316 & 23.9389 & 0.0073 & 1.0947 & 1.1019 & 0.0072 & 1.37 \\
\hline & Isopropanol & 23.7327 & 23.7425 & 0.0098 & 1.1188 & 1.1285 & 0.0097 & 1.02 \\
\hline
\end{tabular}




\section{Appendix D: Equipment List}

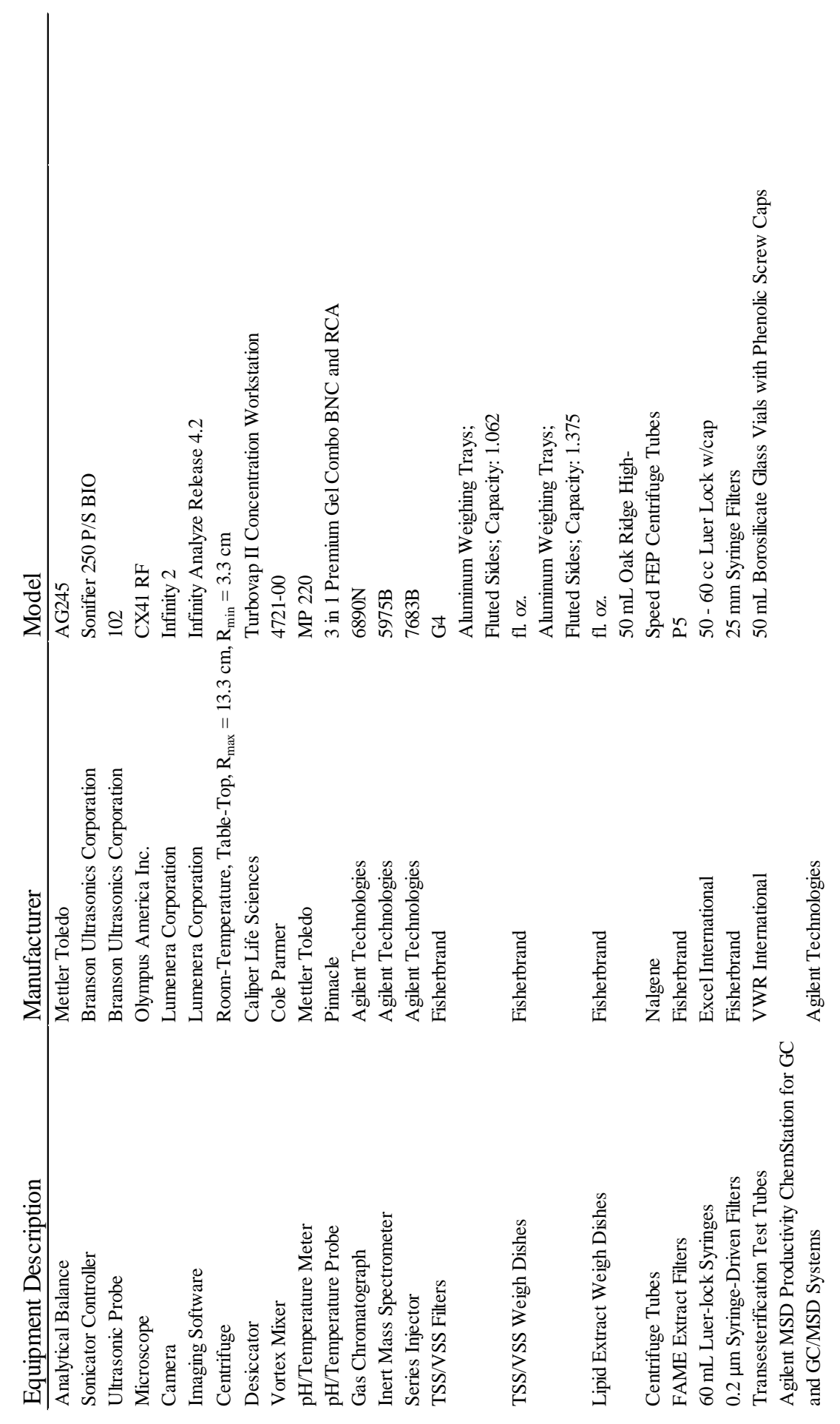

